\title{
Self-Assembled Nanoreactors
}

\author{
Dennis M. Vriezema, Marta Comellas Aragonès, Johannes A. A. W. Elemans, Jeroen J. L. M. Cornelissen, \\ Alan E. Rowan, and Roeland J. M. Nolte* \\ Department of Organic Chemistry, Institute for Molecules and Materials, Radboud University Nijmegen, Toernooiveld 1 , \\ 6525ED Nijmegen, The Netherlands
}

Received September 9, 2004

\section{Contents}

1. Introduction

1445

2. Molecular Nanoreactors

2.1. Capsules and Boxes

2.1.1. Covalent Systems

2.1.2. Noncovalent Systems

2.2. Micelle-Based Systems

2.3. Vesicle-Based Systems

2.3.1. Reactions in or at the Vesicle Membrane

2.3.2. Reactions in the Inner Compartment of Vesicles

3. Macromolecular Nanoreactors

3.1. Polymersomes as Nanoreactors

3.2. Polymer Micelles as Nanoreactors

3.3. Unimolecular Nanoreactors

3.4. Other Polymeric Systems as Nanoreactors

4. Biomacromolecular Nanoreactors

4.1. Protein Cages

4.1.1. Ferritin

4.1.2. Other Protein Cages

4.2. Viruses

4.2.1. Rod-Shaped Viruses

4.2.2. Cage-Structured Viruses

5. Conclusions and Outlook

6. Acknowledgment

7. References

1446

1446

1446

1450

1456

1459

1459

1463

1465

1465

1470

1473

1473

1476

1476

1477

1478

1478

1478

1482

1485

1485

1485

\section{Introduction}

In nature, chemical conversions take place in a confined environment and are closely geared to each other such that the product of one reaction is the substrate or catalyst of the subsequent one. Such a coupling of reactions in time and space (i.e., performing cascade reactions) is of growing interest to chemists as it is expected to increase the efficiency of chemical conversions on scales ranging from the laboratory bench to industrial plants. Nature realizes this coupling and the control and fine-tuning of the products, among others, by using well-defined reaction environments, which vary from nanometer-sized and relatively simple systems, such as enzymes, to micrometer-sized and extremely complex assemblies,

* To whom correspondence should be addressed. Fax: +31 24 3652929. E-mail: r.nolte@science.ru.nl. such as cells. Industry currently uses whole cells as complete factories to produce on a large scale a variety of products. For a synthetic chemist, the coupling of reactions in time and space begins with the design and construction of a confined reaction environment within which the conversion(s) will take place, that is, a reactor. Toward this goal, the cell acts as the primary source of inspiration. Initial attempts to create such reactors involved fully synthetic low molecular weight receptors capable of encapsulating reagents; however, in most cases, the construction of covalent reactors implies complicated multistep synthesis, which is a severe drawback for their application on a larger scale. The development of synthetically more accessible systems demanded a more natural approach, that is, the self-assembly of small molecular components, for example, phospholipids, into discrete, capsule-containing architectures, and vesicles. Although the construction of an artificial cell is the ultimate fantasy, more simple systems such as micelles, vesicles, and other assemblies of molecules may already partly solve the problem. In these simple reactors not only the reaction pathways can be influenced, but in many cases also the size and morphology of the products (e.g., crystals).

The aim of this review is to give an overview of the wide range of nanoreactors that have been constructed from synthetic and biological building blocks using both covalent and noncovalent approaches. The primary focus is on self-assembled systems, varying in size from a few nanometers to tens of micrometers. Not included is the vast area of inorganic compounds, such as zeolites and sol-gels. The self-assembled nanoreactors that are discussed can be considered as the simplest mimics of a cell and contain a cavity in which a chemical reaction can take place. The review is divided into several sections that cover the development of tailor-made nanoreactors, starting from small organic molecular containers expanding to large compartment-containing assemblies. The construction of capsules from low molecular weight compounds by means of covalent synthesis and selfassembly by highly directive and pre-designed interactions, such as hydrogen bonding and metal-ligand coordination, is presented in section 2.1. In subsequent sections (2.2 and 2.3), nanocapsules based on micellar and vesicular assemblies that are built up from low molecular weight molecules will be dis- 


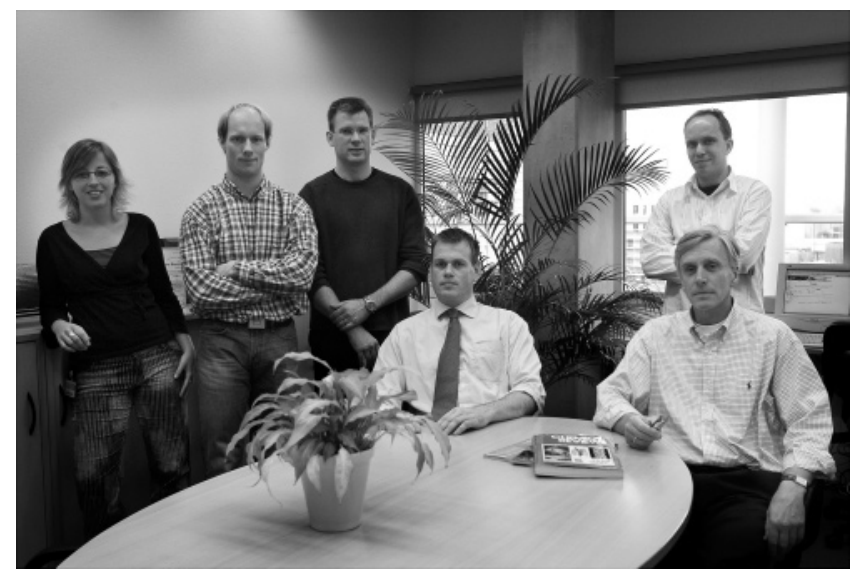

Dennis M. Vriezema (second from left) completed his Chemistry degree at the University of Nijmegen in 1999 and became a researcher in a joint project of this university with the Dutch Research Institute TNO, working on a project to develop a percutaneous glucose monitor. In 2000, he joined the group of Professor R. J. M. Nolte to carry out a Ph.D. project on the aggregation behavior of isocyanide polymers and the development of polymeric nanoreactors. He currently is a postdoctoral fellow in the group of Professor Nolte and is in the process of setting up a spin-off company called Encapson.

Marta Comellas Aragonès (far left) studied Chemistry at the University of Barcelona. After graduating in 2002, she carried out her Masters research at the University of Nijmegen, investigating the assembly of polymer/ protein biohybrids. Currently, she is a Ph.D. student in the group of Professor R. J. M. Nolte, working on the exploration of viral capsids as nanoreactors.

Hans A. A. W. Elemans (standing, right) completed his Ph.D. in Physical Organic Chemistry in 2001 at the University of Nijmegen with Professor R. J. M. Nolte, after which he commenced a postdoctoral position investigating scanning probe techniques in conjunction with the Physics Department and Professor Nolte. Since 2003, he occupies a bridging position between the Solid State Physics and Organic Chemistry groups, where he is conducting research into the self-organization and function of organic molecules at solid-liquid interfaces. For this work, he was awarded a Veni Innovative Research Grant in 2004. His main scientific interests are at the interface of Chemistry and Physics, in particular in the areas of catalysis and self-assembled architectures.

Jeroen J. L. M. Cornelissen (third from left) studied Chemistry at the University of Nijmegen and received his Ph.D. (cum laude) in 2001 on the hierarchical transfer of stereochemical information in synthetic polymers with Professor R. J. M. Nolte. For this work, he was awarded the 2002 IUPAC Prize for Young Chemists and the 2002 SNS Bank Prize. From 2001 to 2002, he worked as a postdoctoral fellow at the IBM Almaden Research Center with Dr. R. D. Miller. Thereafter, he returned to the University of Nijmegen with a Veni Innovative Research Grant to work on structurally defined polymers, biohybrid macromolecules, and protein cages as reactors and supramolecular building blocks.

Alan E. Rowan (seated, left) completed his Ph.D. study at the University of Liverpool in the field of Physical Organic Chemistry in 1990. He then departed to New Zealand for a postdoctoral stage at the University of Otago. In 1993, he returned to Europe to take up a TMR Fellowship at the University of Nijmegen in the field of Supramolecular Chemistry in the group of Professor R. J. M. Nolte. Since then, he became Lecturer, Senior Lecturer, and recently Professor of Molecules and Materials at the University of Nijmegen. His passion is for fine chemistry and good single malts.

Roeland J. M. Nolte (seated, right) received his Ph.D. in Physical Organic Chemistry from the University of Utrecht (1973), where he stayed and became Assistant Professor and then Associate Professor. In 1981, he was a visiting scientist at UCLA in the group of Professor Donald J. Cram. In 1987, he moved to the University of Nijmegen and became a Full Professor of Organic Chemistry, and since 1994 he has also been a part-time Professor of Supramolecular Chemistry at the Eindhoven University of Technology. In 2003, he was awarded the first Royal Netherlands Academy of Arts and Science Chair in Chemistry. His research interests span a broad range of topics at the interfaces of Supramolecular Chemistry, Macromolecular Chemistry, and Biomimetic Chemistry. In his work, he focuses on the design of catalysts and (macro) molecular materials. He and his group have published ca. 450 scientific papers. cussed, whereas section 3 covers the construction of nanoreactors from macromolecular building blocks. Finally, in section 4 we will give an overview of the most recent developments in the use of viruses as nanocontainers and-reactors. We have focused in our review on developments in the past decade, covering the fields of organic and macromolecular chemistry as well as the emerging area of biomacromolecules (e.g., viruses). All important articles in these fields are highlighted, and where appropriate the foundation papers from relevant earlier work are included.

\section{Molecular Nanoreactors}

\subsection{Capsules and Boxes}

\subsubsection{Covalent Systems}

The most efficient catalysts known are nature's enzymes, whose tremendously high efficiency and selectivity have always been the drive for chemists to create synthetic catalytic systems that approach a similar superior activity and selectivity. In this respect, the general architecture of an enzyme has been a key of inspiration for the development of artificial supramolecular catalytic systems. Many of the systems of this type rely on principles reported already 60 years ago by Pauling in his fundamental theories of enzyme catalysis with respect to transition states and molecular recognition ${ }^{1}$ and are therefore commonly referred to as artificial enzymes. ${ }^{2}$ The most important feature of an enzyme is that the activated complex is stabilized to a larger extent than the enzyme-substrate complex itself, by the presence of additional binding interactions in the transition state of the reaction. In other words, enzymes can be considered complementary in structure to the transition state of the reaction they catalyze. The enzyme's binding properties, coupled with catalytic functionalities strategically placed with the active site, decrease the activation energy for the reaction. ${ }^{3,4}$ To be an efficient enzyme mimic, the designed molecule therefore needs to possess a binding cavity or site that is able to selectively recognize and bind a desired substrate, which in the next step has to be converted at a catalytic center in its direct proximity. Finally, and in the design of many artificial enzymes this has proven to be a major bottleneck, the catalyst should be able to release the converted substrate and have the ability to be regenerated; that is, turnover has to occur.

Using nature's enzymes as a blueprint, many approaches to synthesize low molecular weight catalysts, which contain a substrate binding site that recognizes substrates next to an active site, have been described in the literature. ${ }^{5}$ The first, simple examples of enzyme mimics were crown ethers and cryptands with catalytically active functionalities attached in the correct position near the reacting group of a complexed substrate.$^{6-9}$ From the early 1970s on, cyclodextrins (CDs), naturally abundant cavity molecules, have been extensively used as binding sites in supramolecular catalysts. ${ }^{10-13} \mathrm{~A}$ myriad of functionalized cyclodextrins have shown to be selective catalysts for ester hydrolysis. ${ }^{14-17} \mathrm{~A}$ 


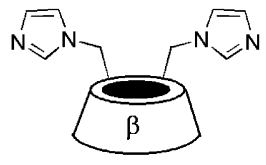

1

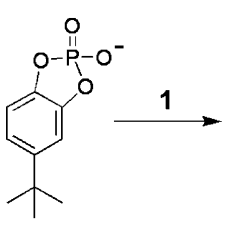

2

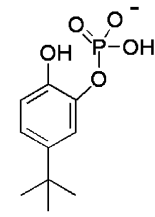

$3 a$

(a)

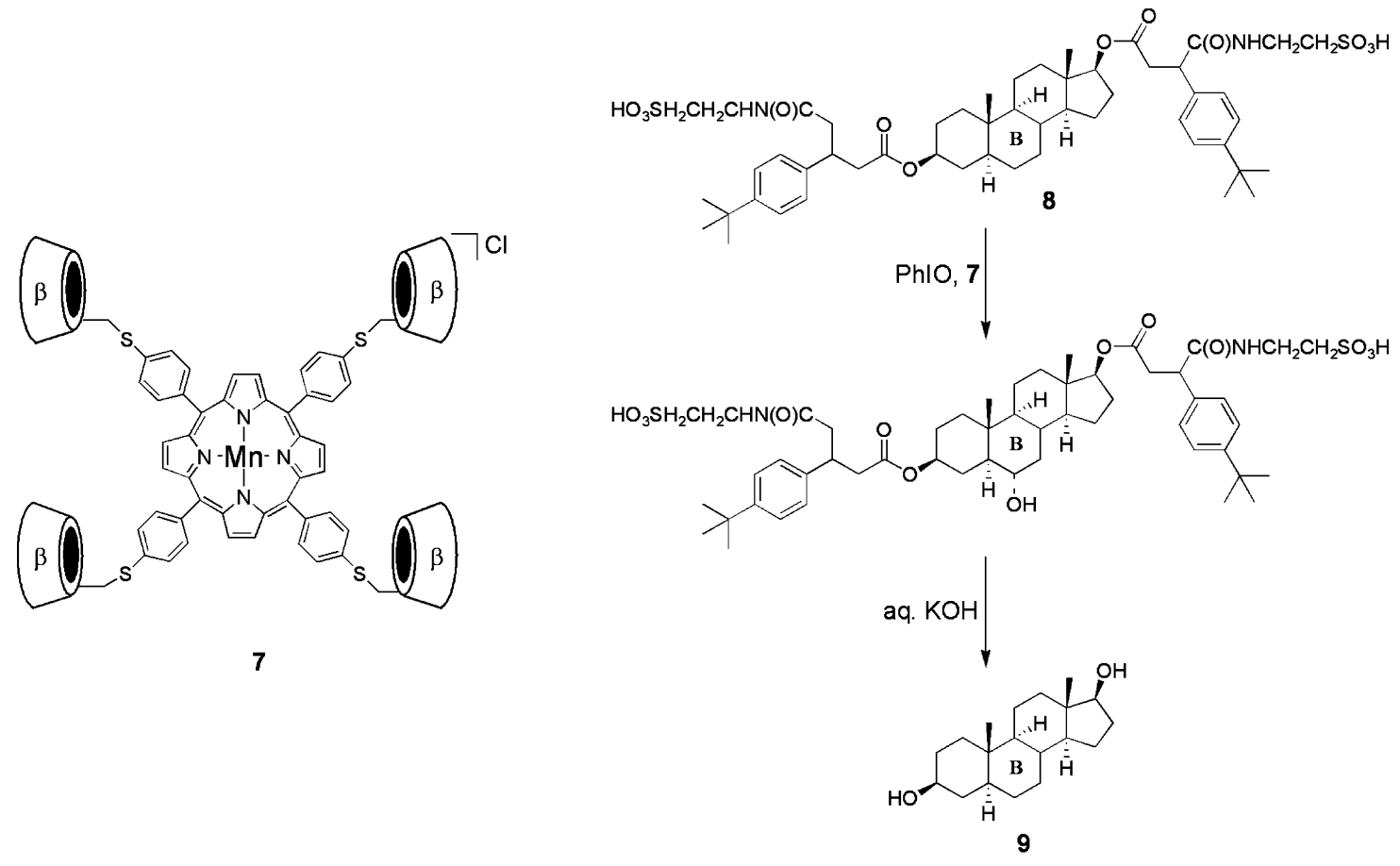

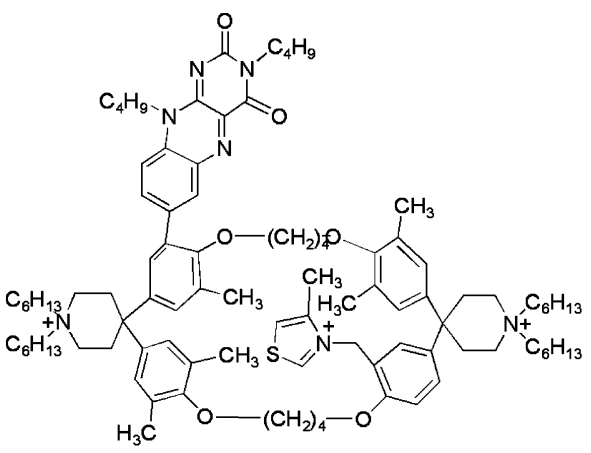

4

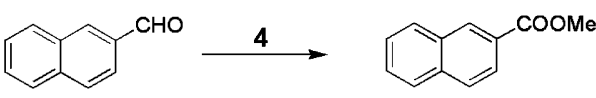

5

(b)

(c)

Figure 1. Examples of artificial enzymes: (a) Breslow's ribonuclease A mimic which catalyzes the hydrolysis of cyclic phosphodiesters, (b) Diederich's pyruvate oxidase mimic which catalyzes the oxidation of naphththalene-2-carbaldehyde, and (c) Breslow's manganese porphyrin tetra-cyclodextrin receptor which regio- and stereoselectively hydroxylizes steroid derivatives.

representative example is Breslow's mimic of the enzyme ribonuclease A, ${ }^{14,18}$ which works according to principles of acid-base catalysis (Figure 1a). In this mimic, two imidazole rings are connected to the primary side of a $\beta$-CD, and the resulting cavity molecule 1 binds and selectively hydrolyzes cyclic phosphor diester 2. Apart from a 120-fold rate enhancement as compared to the uncatalyzed solution reaction using $\mathrm{NaOH}$ as a base, a greater than 99\% selectivity for one of the two possible products (i.e., 3a) was observed. Following a similar approach,
Diederich covalently attached a thiazolium and a flavin moiety to a cyclophane cavity, creating a synthetic pyruvate oxidase mimic 4 (Figure 1b). ${ }^{19,20}$ The system is capable of catalyzing, on a truly preparative scale, the conversion of complexed naphththalene-2-carbaldehyde 5 into the methyl ester $\mathbf{6}$. During the reaction, the flavin residue is reduced, and for turnover to occur it is reoxidized by regeneration in an electrochemical cell.

Many other examples are known in which a transition metal catalyst is covalently attached to one or 
more substrate binding cavities. For example, iron porphyrins connected to one $\mathrm{e}^{21,22}$ or more ${ }^{21-24} \mathrm{CDs}$ have been applied as oxidation catalysts with varying efficiencies and selectivities of substrates that are bound in the CD cavity. Different types of cavitycontaining catalysts are metal-ligand systems connected to cyclocholates, ${ }^{25,26}$ cyclophanes, ${ }^{27-29}$ calixarenes, ${ }^{30}$ clips, ${ }^{31-34}$ and other concave binding sites. ${ }^{35-38}$ An artificial enzyme system which perfectly illustrates the possibility to achieve high selectivity is the manganese porphyrin $\mathbf{7}$, which possesses four covalently linked peripheral $\beta$-CDs (Figure 1c). ${ }^{39-43}$

In the presence of iodosyl benzene, this tetramer is capable of selectively epoxidizing or hydroxylating stilbene derivatives. Its superior selectivity is best illustrated by the fact that it can regio- and stereoselectively oxidize the unactivated $6-\mathrm{CH}_{2}$ position in the B-ring of steroid derivative 8. The selectivity relies on a precise positioning of this methylene group directly over the porphyrin metal center, caused by the encapsulation of the two tert-butylphenyl groups of the substrate by two trans-positioned CD cavities, as was concluded from molecular modeling calculations. As a result of concomitant hydrolysis of the ester groups during the reaction, product 9 was obtained in a yield of $40 \%$.

The majority of the examples discussed above refer to supramolecular catalysts which convert a single bound substrate. In recent years, however, new receptors have been developed that are designed in such a way that they can simultaneously complex two or even more substrates, and in addition promote a reaction between them. In many cases, the binding site is modified in such a way that a reaction chamber is created which encapsulates guest molecules, confines them, and effectively shields them from their environment, generally the solvent. As a result, the transition state of more than one simultaneously captured substrate in a bimolecular reaction can be stabilized. The appropriate catalysis of bimolecular reactions is, however, not trivial, because apart from binding the two substrates it is also necessary to orient them in the correct position for the reaction to occur effectively. ${ }^{44}$ On the other hand, in such a way a careful design of the host might allow for a geometrical restriction of the bound substrates and a concomitantly enforced stereo- or regioselectivity in product formation. A frequently occurring example is the acceleration of cycloaddition reactions under encapsulation conditions. ${ }^{45}$ When both partners of the cycloaddition reaction are oriented in the correct geometry within the host, the latter acts as the template for a transition state analogue, on which also the development of catalytically active imprinted polymers, ${ }^{46}$ catalytic antibodies, ${ }^{47}$ and the recently developed selection of a catalyst from a dynamic combinatorial library (vide infra) are based. A serious limitation of the use of templating hosts, however, is that they are in many cases not truly catalytic. Because their design generally relies on binding the reactants in a geometry that resembles that of the eventual products, the latter almost inevitably bind to the host with a similar strength or even stronger, thereby preventing effective turnover by product in-
Scheme 1. 1,3-Dipolar Cycloaddition between Propargylammonium 12 and Azidoethylammonium 11 Catalyzed by Cucurbit[6]uril 10
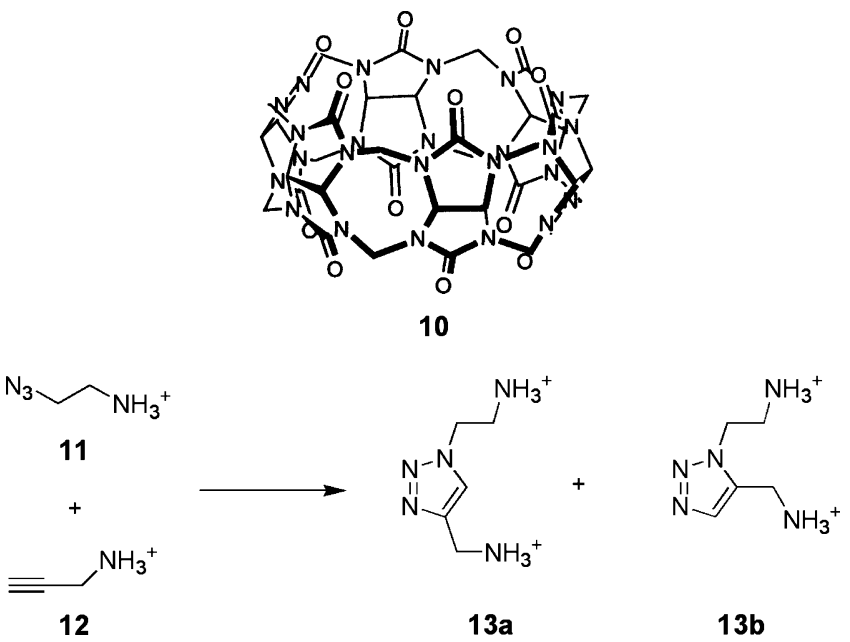

hibition. Approaches to address this problem include a careful design of the binding sites of the capsule, in such a way that they bind the products weaker than the reactants, or by aiming for reaction products that are too small or too large to fit within the host. In particular, the latter approach has proven to be extremely versatile in cases where the hosting capsule itself can be reversibly assembled and disassembled (see section 2.1.2).

Initially, CDs were utilized as catalysts of DielsAlder reactions in water, ${ }^{48,49}$ in one case revealing Michaelis-Menten kinetics with saturation occurring at high substrate concentrations, ${ }^{50}$ whereas inhibition of the reaction occurred in the presence of a hydrophobic dienophile. In a related study, significant changes in diastereoselectivity of the reaction could be achieved in the presence of CD catalysts. ${ }^{51} \mathrm{An}$ other family of macrocycles that have been applied as nanoreactors more recently are cucurbituril derivatives. ${ }^{52,53}$ The internal cavity of the hexameric glycoluril derivative cucurbit[6] uril $\mathbf{1 0}$ is sufficiently large to accommodate aliphatic and aromatic fivemembered rings, and consequently it was investigated as a nanoreactor for 1,3-dipolar cycloaddition reactions. ${ }^{54}$ The uncatalyzed reaction between propargylammonium 11 and azidoethylammonium 12 yields a mixture of two regioisomeric diazole adducts $\mathbf{1 3 a}$ and 13b, which are formed in equal amounts by a standard concerted process (Scheme 1). In the presence of a catalytic amount of $\mathbf{1 0}$, the rate of this reaction was accelerated $55000 \times$. In addition, the 1,4-disubstituted product was exclusively formed. These results are explained by assuming the formation of a transient ternary complex between cucurbituril and the both reactants. The reactants are bound simultaneously by electrostatic interactions between the charged ammonium groups and the urea oxygen atoms lining the portals of $\mathbf{1 0}$, with the acetylene and azide substituents extending into the core of the host. The reactive groups are perfectly aligned with respect to each other, and a rapid reaction occurs. Not unexpectedly, the release of the pseudorotaxane product, which has a higher affinity for the host than each of the both reactants, is the 
rate-limiting step. In more recent work, Tuncel and Steinke have shown that this cucurbituril-catalyzed Diels-Alder reaction can also be applied in the synthesis of polyrotaxanes by using diazides and diacetylenes as the substrates. ${ }^{55,56}$

The octameric analogue cucurbit[8] uril, which has a cavity with a size comparable to that of $\gamma$-CD, can accommodate two aromatic guest molecules. The cavity was used as a nanoreactor in the [2+2]photodimerization of $(E)$-diammoniumstilbene in aqueous solution. ${ }^{57}$ Apart from a large rate acceleration as compared to a similar reaction catalyzed by $\gamma-\mathrm{CD}$ ( 0.5 vs $72 \mathrm{~h}$ for $\gamma$-CD), a considerable stereoselectivity was observed resulting in a ratio between the synand anti-adducts of 95:5. In addition, no isomerization, which is usually observed for stilbene derivatives, was observed during the reaction. The observed results are explained by assuming a stabilization of both guest molecules in a favored geometry in which the olefinic groups are oriented in a parallel fashion within the substrate binding cavity.

Sanders and co-workers were among the first to synthesize more sophisticated hosts for the use as nanoreactors, viz., cyclic zinc porphyrin hosts, which possess convergent binding sites that can specifically complex two reaction partners in close proximity by providing them with ligands that coordinate to the porphyrin zinc ions. In the presence of trimer 14a, the Diels-Alder reaction between pyridine-functionalized maleimide $\mathbf{1 5}$ and furan derivative $\mathbf{1 6}$ appeared to occur with a 200 -fold increase in initial rate as compared to when the reaction was carried out in the absence of $\mathbf{1 4 a}$ (Scheme 2). ${ }^{58}$

In addition, when using $14 a$, the exo-adduct 17 was the only product formed. These results can only be explained by assuming a simultaneous binding of $\mathbf{1 5}$ and 16 within 14a, which positions the starting materials in the correct geometry for producing the exo-product exclusively. Acceleration of the reaction occurs simply by virtue of the binding of the reactants by 14a: its favorable enthalpic gain compensates for the highly unfavorable entropy of activation. Further evidence for the occurrence of the reaction within the cavity of 14a was provided by the fact that in the presence of tripyridyltriazine, a guest that is bound extremely strong within $\mathbf{1 4 a}$, the Diels-Alder reaction was completely inhibited. As expected, the major drawback that prevents 14a from acting as a true catalyst is the occurrence of product inhibition, because $\mathbf{1 7}$ is complexed by $\mathbf{1 4}$ far more favorably than the starting materials. The formation of the endo-adduct is disfavored because of a poor fit of its transition state within the cavity of $\mathbf{1 4 a}$. However, upon contraction of the cavity, the stereochemistry of the reaction could be completely reversed. ${ }^{59}$ Host $\mathbf{1 4 b}$, which contains two ethyne instead of butadiyne bridges, incorporates two different $\mathrm{Zn}-\mathrm{Zn}$ distances, viz., $15 \AA$, as in the case of $\mathbf{1 4 a}$, and $12 \AA$. The latter distance is more complementary to binding of the endo-adduct 18, which is formed exclusively upon reaction of $\mathbf{1 5}$ and 16. Similar rate accelerations using cyclic metalloporphyrin trimers were observed in the case of hetero Diels-Alder reactions between pyridyl dienes and nitrosopyridines to yield oxa-

\begin{abstract}
Scheme 2. Controlled Direction of a Diels-Alder Reaction by Means of the Geometrical Constraints of Cyclic Porphyrin Hosts
\end{abstract}
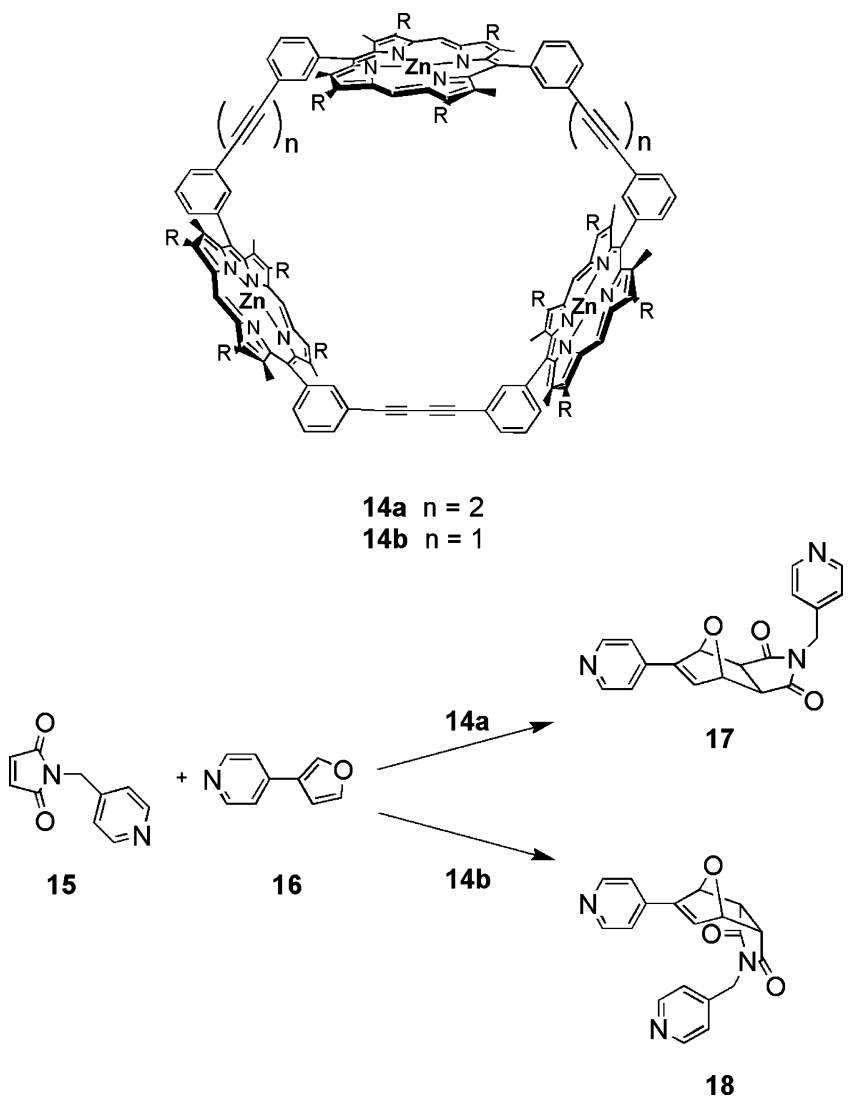

zines. ${ }^{60-62}$ Host 14a was turned into a true catalyst when it was used in a catalytic acyl transfer reaction. ${ }^{63}$ Upon hosting 4-(hydroxymethyl)pyridine and $N$-acetylimidazole, the substrates are perfectly oriented to undergo a transacylation reaction. As compared to the uncatalyzed reaction, 14a increased the initial rate of the reaction 16 -fold. In contrast to the case of the Diels-Alder reactions, however, the products are now coordinated to $\mathbf{1 4 a}$ by only a single zinc-ligand interaction, resulting in a binding affinity of the product which is comparable to those of the substrates, allowing turnover to occur.

In the above examples, molecular capsules are utilized to induce cycloaddition reactions between two simultaneously complexed substrates. In the reverse approach, encapsulation has also proven to be an extremely powerful tool to prevent reactions by the trapping of reactive intermediates. ${ }^{64}$ The inclusion of reactive compounds in a confined space has thus become a means to control their reactivity toward compounds other than themselves. For example, Cram's hemicarcerand-type containers can incarcerate $o$-benzyne or cyclobutadiene and stabilize them by protecting them for dimerization. ${ }^{65-67}$ Recently, Warmuth et al. reported the generation and stabilization of cycloheptatetraene inside the cavity of a hemicarcerand, ${ }^{68,69}$ and reactive cations have been stabilized by trapping them inside a tetrahedral gallium-ligand cluster. ${ }^{70}$ Similarly, gases such as $\mathrm{NO}_{2}$ have been chemically "fixated" and utilized as stable nitrosation reagents by encapsulating them within calixarenes. ${ }^{71-73}$ 


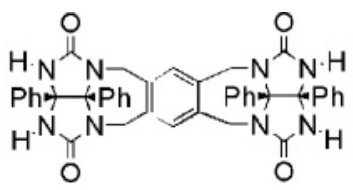

19

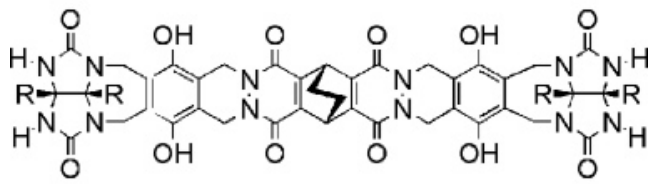

$20 \mathrm{R}=4-n$-heptylphenyl

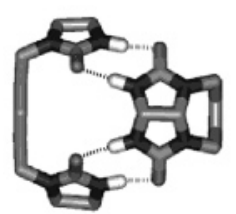

19-19

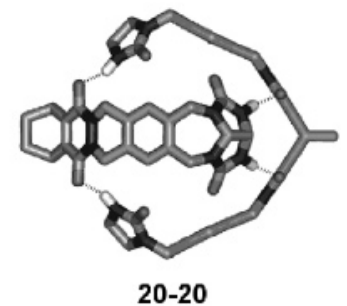

(b)
Figure 2. Glycoluril-based capsules which are self-assembled by hydrogen-bonding interactions: (a) a "tennis ball" and (b) a "softball". In the computer modeled structures, some of the substituents and hydrogen bonds have been omitted for clarity. (Reproduced with permission from ref 74. Copyright 2002 Wiley.)

\subsubsection{Noncovalent Systems}

Covalently built capsules have proven their value as catalysts of a wide range of chemical reactions, and in several cases impressive efficiencies and selectivities have been achieved (see the examples described in section 2.1.1). However, as the scale and complexity of the target products of their reactions increase, designing and preparing capsule-shaped catalysts in a covalent fashion rapidly becomes very difficult, involving costly multistep syntheses that often produce only milligram yields of material. ${ }^{19,32,59}$ Moreover, the resulting catalysts are not so versatile because they are often highly specific to only a limited number of reactions. ${ }^{54,58,63-69}$ It is for this reason that in the past decade a shift has been observed in the approach to construct these catalysts, from traditional covalent chemistry to multicomponent selfassembly of relatively small complementary building blocks. Self-assembled capsules are receptors with enclosed cavities that are formed through reversible, noncovalent interactions between two or more complementary subunits. Typically, such capsules selfassemble through hydrogen-bonding and metalligand interactions. ${ }^{74,75}$ Hydrogen-bonding interactions are highly directional and specific, whereas metalligand bonds are generally much stronger, yielding more robust self-assembled structures. In this section, self-assembled capsules that can function as nanoreactors will be discussed.

The group of Rebek has become one of the leading groups in the exploration of self-assembly of complementary concave receptors into a myriad of hydrogenbonded capsular species. ${ }^{76-79}$ The first capsule, the so-called "tennis-ball" 19-19 (Figure 2a), consists of two bis-glycoluril units which self-assemble into a closed cavity.

The self-assembly is governed by the specific curvature encoded within the building block, and the concomitant formation of eight hydrogen bonds between the self-complementary $\mathrm{C}=\mathrm{O}$ and $\mathrm{N}-\mathrm{H}$ functions of the two subunits. ${ }^{80}$ The inner volume of the capsule $\left(\sim 50 \AA^{3}\right)$ is rather small and only allows for the encapsulation of small molecules such as methane and noble gases. To complex larger guests, the spacers between the glycoluril moieties were varied, generating a number of dimeric and higher order capsules with inner compartments of varying sizes. By comparing the binding properties of these capsules, it turned out that the encapsulation process was largely determined by the size and shape of the guest. An extensive study toward the understanding of the factors that govern the binding of guests within Rebek's capsules revealed the " $55 \%$ occupance" rule, which implies that in the absence of specific intermolecular interactions between the guest and the capsule the binding is most efficient if only $55 \%$ of the available inner space is filled. ${ }^{81}$

A typical example of a larger capsule generated by the assembly of extended subunits is the "molecular softball" 20-20, in which the glycoluril moieties are connected across seven fused rings (Figure $2 \mathrm{~b}$ ). The central ring contains an ethylene bridge function that encodes the extra curvature that is required for the specific formation of a hydrogen-bonded dimer, resulting in a capsule which is now held together by a seam of 12 hydrogen bonds. The complexation of guest molecules within the softball is, interestingly, an entropy-driven process, which was concluded from the fact that the association constants increase with temperature. This was unexpected, because most host-guest complexation processes in organic solution are entropically unfavorable and enthalpydriven. Upon binding of a guest, for example, adamantane or ferrocene, in 20-20, two molecules of solvent that are present in the cavity are released, resulting in a favorable increase in entropy. NMR studies also revealed that the softball was capable of encapsulating two aromatic solvent molecules, ${ }^{82,83}$ and this propensity stimulated the researchers to utilize the "softball" as a microreactor for bimolecular reactions. An example is depicted in Figure 3a, viz., the Diels-Alder reaction between $p$-benzoquinone 21 and cyclohexadiene $22 .{ }^{84,85}$ In $p$-xylene- $d_{10}$ solution, the rate of this reaction is 200 times faster in the presence of the 20-20 capsule than in its absence. This was explained by assuming that both starting materials are complexed within the capsule and, because of their close proximity, the rate of reaction is enhanced. Due to the restricted space inside the capsule, the endo-isomer $\mathbf{2 3}$ is favored as the exclusive product. In contrast, if 1,4-naphthoquinone, a molecule that does not fit in the capsule, was used as the dienophile, the reaction was not accelerated. Addition of a nonreactive guest that effectively competes for encapsulation with the reactants also was found to prevent rate acceleration.

A major deficit of the reaction, as expected, is that effective turnover is prevented due to product inhibi- 


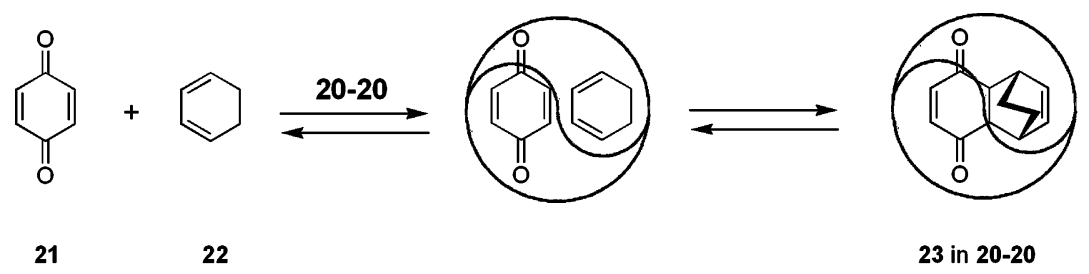

(a)
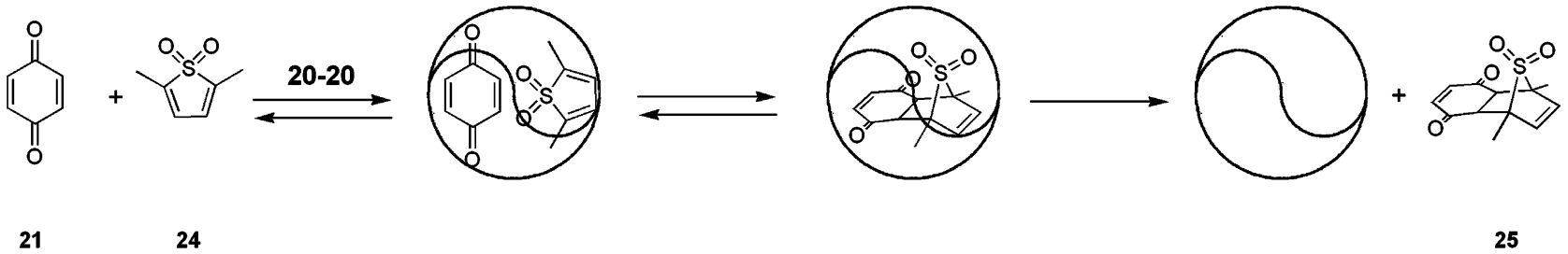

21

24

(b)

Figure 3. (a) The Diels-Alder reaction between cyclohexadiene and $p$-benzoquinone accelerated by microreactor 20-20, which is needed in stoichiometric quantities due to product inhibition. (b) The Diels-Alder reaction between 2,5-dimethyl thiophene dioxide and $p$-benzoquinone catalyzed by the microreactor 20-20; in this case, product release makes 20-20 available for catalyzing further reactions.

tion, because the resulting adduct fits perfectly within the capsule $\left(K_{\mathrm{a}}>10^{5} \mathrm{M}^{-1}\right)$. To make the capsule a true catalyst, product release is essential, and this was accomplished by using 2,5-dimethyl thiophene dioxide $\mathbf{2 4}$ as the diene in the cycloaddition reaction with $p$-benzoquinone. The capsule has a poor affinity for the resulting Diels-Alder product 25, which, assisted by the ability of the capsule to disassemble and re-assemble, can be replaced by new reactants (Figure $3 \mathrm{~b}) .86$

Capsule 26-26, which is composed of a dimer of complementary resorcinarene units stabilized by 12 hydrogen bonds (Figure 4), ${ }^{88}$ can function as a reaction chamber in which the 1,3-dipolar cycloaddition between phenylacetylene $\mathbf{2 7}$ and phenyl azide $\mathbf{2 8}$ is accelerated. ${ }^{89}$ Whereas in the absence of $\mathbf{2 6 - 2 6}$ it takes years for this reaction to complete, in the presence of the capsule it occurs within a few days. In addition, in the latter case absolute regioselectivity is observed in the sense that the 1,4-adduct $\mathbf{2 9}$ is formed exclusively. Because the product is an excellent guest for 26-26, the reaction clearly suffers from product inhibition and no true catalysis is accomplished.

During the past decade, several research groups have reported the construction of discrete cagelike architectures by means of metal-ligand selfassembly. ${ }^{90-94}$ In contrast to hydrogen-bonding motifs, which are linear in nature and thus require the presence of curvature elsewhere in the building block for a capsule to be formed, the specific coordination angles between ligands and metal centers can be used themselves as codons for curvature. Fujita and co-workers have elaborated ${ }^{95}$ on the conceptual design and synthesis of well-defined cages by multicomponent transition metal-mediated self-assembly processes for the use as reaction chambers for several types of bi- and multimolecular reactions. The building blocks of these highly symmetrical metallocapsules are simple triangular heterocyclic ligands and cis-enforced square-planar Pd-and Pt-complexes. Coordination cage $\mathbf{3 0}$, which is the single product of a spontaneous self-assembly process of four tris(4-

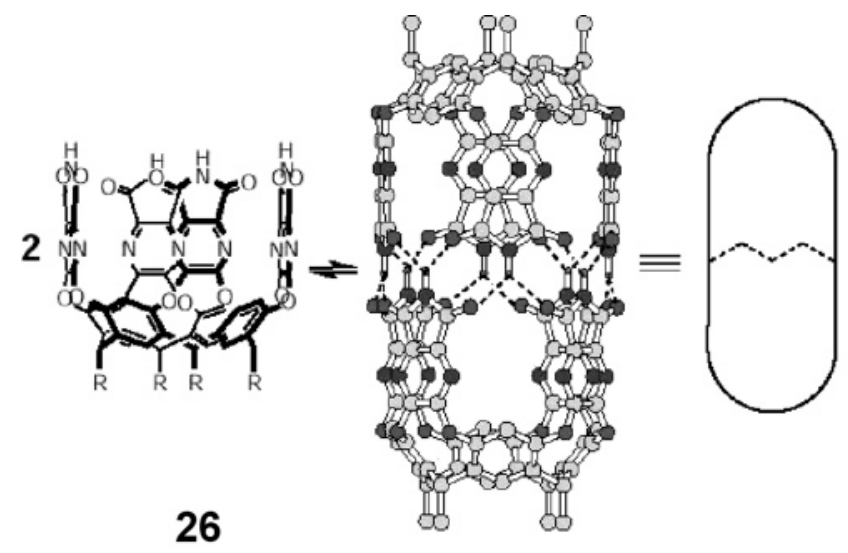

26-26

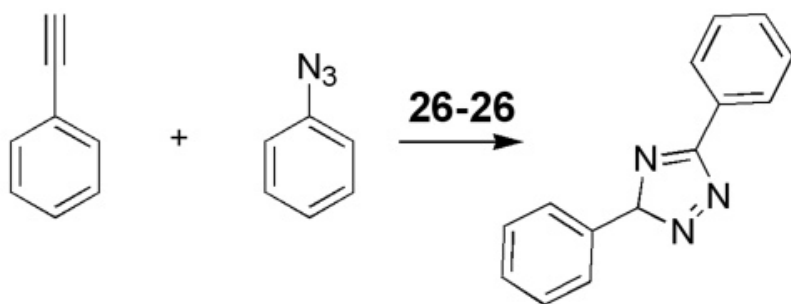

$27 \quad 28$

29

Figure 4. Capsule 26-26, which is self-assembled from two resorcinarene subunits, and the 1,3-dipolar cycloaddition reaction that is accelerated by this capsule. (Adapted with permission from Figure 1 from ref 87. Copyright 2002 National Academy of Sciences.)

pyridyl)triazine ligands and six palladium complexes, has an inner compartment with a volume of about $500 \AA^{3}$ and is capable of encapsulating a variety of neutral organic molecules in aqueous environments (Figure 5). ${ }^{96-100}$

For this reason, the capsule was expected to be an effective mediator for phase-transfer catalysis reactions in water. In the absence of cage 30, the Wacker 


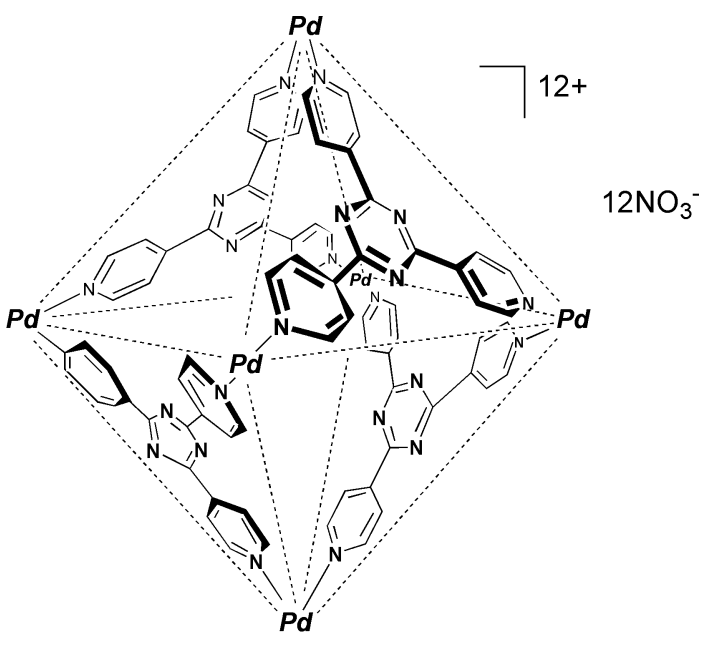

30

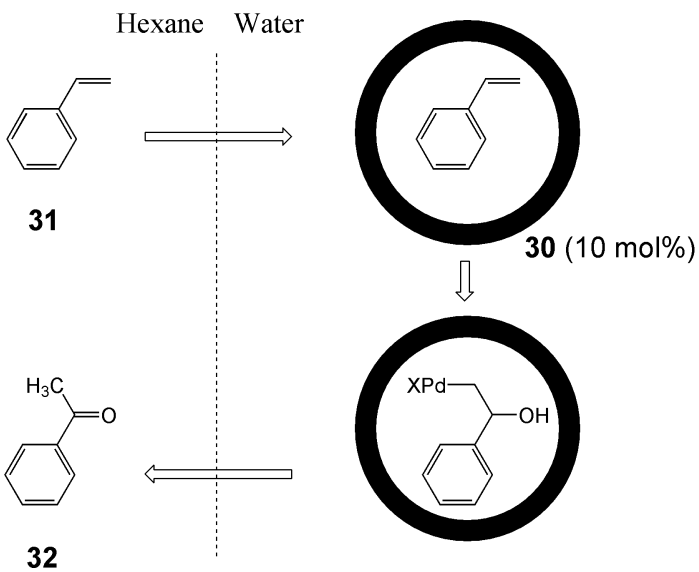

Figure 5. Schematic representation of the Wacker oxidation of styrene to yield acetophenone in a two-phase system, in which capsule $\mathbf{3 0}$ plays the role of a phase-transfer catalyst. (Adapted with permission from Chart 1 from ref 102. Copyright 2001 American Chemical Society.)

oxidation of styrene $\mathbf{3 1}$ to acetophenone $\mathbf{3 2}$, catalyzed by $[\mathrm{Pd}(\mathrm{en})]\left(\mathrm{NO}_{3}\right)_{2}$ (en = ethylenediamine) in water, proceeded only to a small extent (4\%) (Figure 5). In the presence of a catalytic amount of $\mathbf{3 0}$, however, a dramatic increase in the yield of acetophenone to $82 \%$ was observed. ${ }^{101}$ When the reaction was carried out in the presence of 1,3,5-trimethoxybenzene, a molecule that effectively competes with styrene for encapsulation by $\mathbf{3 0}$, the acetophenone production dropped to $3 \%$.

The same coordination cage has been used as a reaction chamber for the bimolecular [2+2] photodimerization of bulky olefins. ${ }^{103}$ Upon irradiation of acenaphthylene $\mathbf{3 3}$ in the presence of $\mathbf{3 0}$ in water, the syn-dimer 34 was formed exclusively in a yield of $>98 \%$ (Figure $6 a$ ).

The presence of the cage appeared to be essential for the reaction to occur and, in addition, for achieving the high stereoselectivity. The cage also turned out to be a catalyst with high regioselectivity, as was evidenced by the [2+2] photodimerization of 1-methylacenaphthylene 35, which almost exclusively afforded the head-to-tail syn-isomer $\mathbf{3 6}$ in $>98 \%$ yield (Figure 6b). In the absence of the cage, no reaction occurred at all.

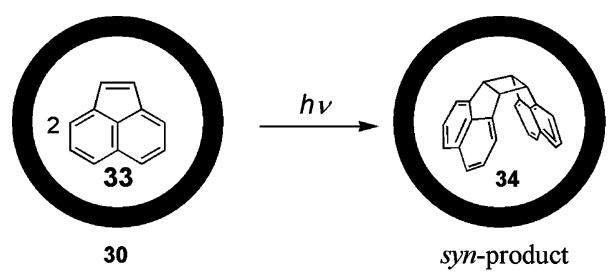

(a)

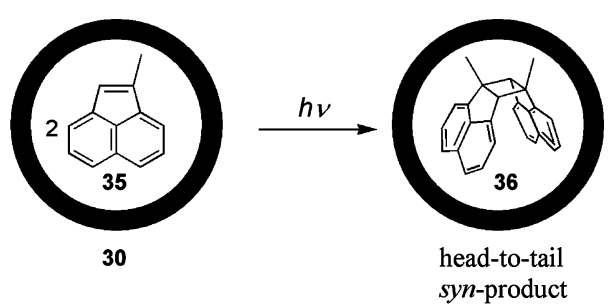

(b)

Figure 6. Schematic representation of the photodimerizations of (a) acenaphthylene and (b) 1-methylacenaphthylene by metallo-cage $\mathbf{3 0}$.

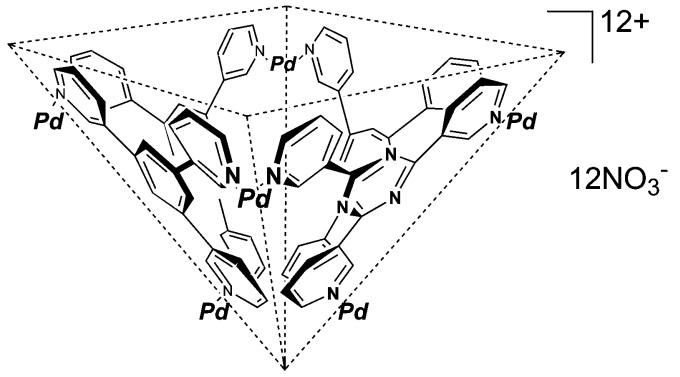

37

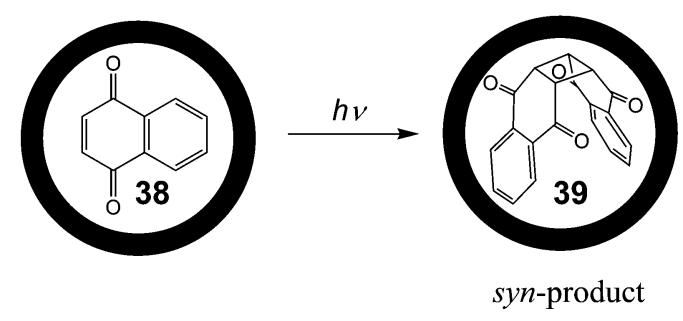

Figure 7. Schematic representation of the photodimerization of 1,4-naphthoquinone by the half open metallocage 37. (Adapted with permission from Chart 1 from ref 102. Copyright 2001 American Chemical Society.)

The photodimerization of naphthoquinones was most effectively catalyzed by the half open coordination cage 37 (Figure 7). 104,105 The dimerization of 1,4naphthoquinone 38 yielded the syn-dimer 39 in $>98 \%$ yield, whereas the same reaction in benzene in the absence of $\mathbf{3 7}$ preferentially gave the anti-dimer. The regioselectivity of the photodimerization of 2-methylnaphthoquinone was slightly higher when catalyzed by 37 as compared to $\mathbf{3 0}$ (96\% and $78 \%$ headto-tail product, respectively). In addition, it could be remotely controlled by placing a substituent on the naphthalene ring, because 5-methoxynaphthoquinone was photodimerized by $\mathbf{3 7}$ with a $79 \%$ head-totail selectivity.

Stimulated by the successful homodimerization reactions, cage $\mathbf{3 0}$ was also explored as a catalyst in the cross-photodimerization of acenaphthylenes and naphthoquinones (Figure 8). ${ }^{106}$ 


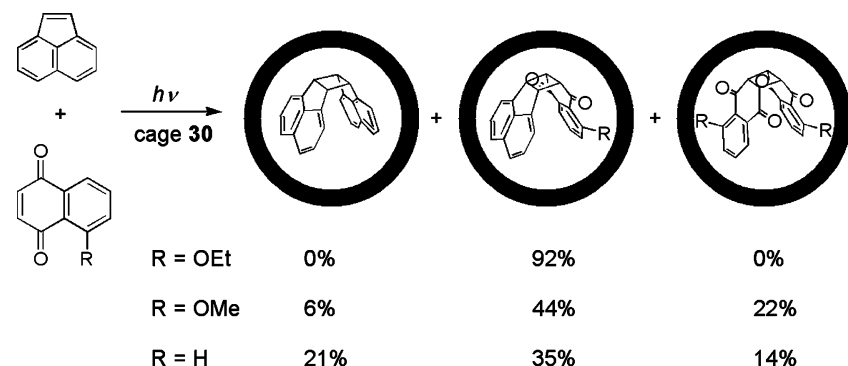

Figure 8. Product distributions of cross-photodimerization reactions of acenaphthylene and 1,4-naphthoquinone derivatives catalyzed by metallo-cage $\mathbf{3 0}$.

In this respect, managing the selectivity between the cross- and homo-coupling reactions is a big challenge because it requires the selective encapsulation of two different olefins within 30. This pairwise selectivity could be nicely controlled by a careful choice of the steric restrictions of the substrates within the capsule: only when a 5-ethoxysubstituent was present on the naphthoquinone was the syn cross-dimer formed exclusively and in high yield. In case of a 5-methoxy, or in the absence of a substituent, also homodimers were formed. A striking result was obtained when acenaphthylene and $N$-benzylmaleimide, an otherwise photochemically inert substrate, could be coupled in the presence of $\mathbf{3 0}$, yielding only the syn-product in $97 \%$.

Fujita and his group have utilized metallo-capsules to stabilize molecules in an unfavorable conformation. ${ }^{98}$ Upon treating an aqueous solution of $\mathbf{3 0}$ with a solution of $4,4^{\prime}$-dimethylazobenzene (cis:trans $=1: 6$ ) in hexane, 2 equiv of selectively the cis-isomer of the guest was encapsulated. In addition, the cis-azobenzene molecules are considerably stabilized by their encapsulation, because exposing the complex to visible light for several weeks did not result in isomerization to the thermodynamically favored transproduct. In a similar fashion, Rebek has used the hydrogen-bonded capsule 26-26 (see Figure 4) to stabilize a tertiary anilide in its $Z$-configuration. ${ }^{107}$

Encapsulation can stabilize labile oligomers, which are formed in situ by the reaction of smaller molecular components. An illustrative example is the use of coordination cages as confined reaction chambers for the condensation of trialkoxysilanes. ${ }^{108}$ Cage $\mathbf{3 0}$ allows the exchange of phenyltrimethoxysilane monomers 40 in aqueous solution, and upon heating the mixture, a cyclic siloxane trimer $\mathbf{4 1}$ was formed in a "ship-in-a-bottle" fashion within the capsule of $\mathbf{3 0}$ in a yield of $92 \%$ (Figure 9a). Whereas in the absence of $\mathbf{3 0}$ the cyclic trimer is only a kinetic, short-lived intermediate that is rapidly converted to thermodynamically more stable cyclic tetramers and further condensed products, the complex of $\mathbf{3 0}$ and the trimer was found to be stable for more than a month in neutral and even the strongly acidic conditions $(\mathrm{pH}$ $<1$ ) required for its isolation.

The oligomerization of trialkoxysilanes could be controlled in an even more sophisticated fashion, 102 following a concept named "cavity-directed synthesis", because it relies on using predesigned cavity sizes and geometries to properly preorganize starting materials and stabilize reaction products. Cages $\mathbf{3 0}$ (Figure 5), 37 (Figure 7), and 42 (Figure 9), which vary in shape and size, were used in oligomerization reactions of 2-naphthyltrimethoxysilane. In the presence of tubelike cage 42, this substrate was only hydrolyzed to its silanol derivative, which, because of its encapsulation within 42, remained remarkably

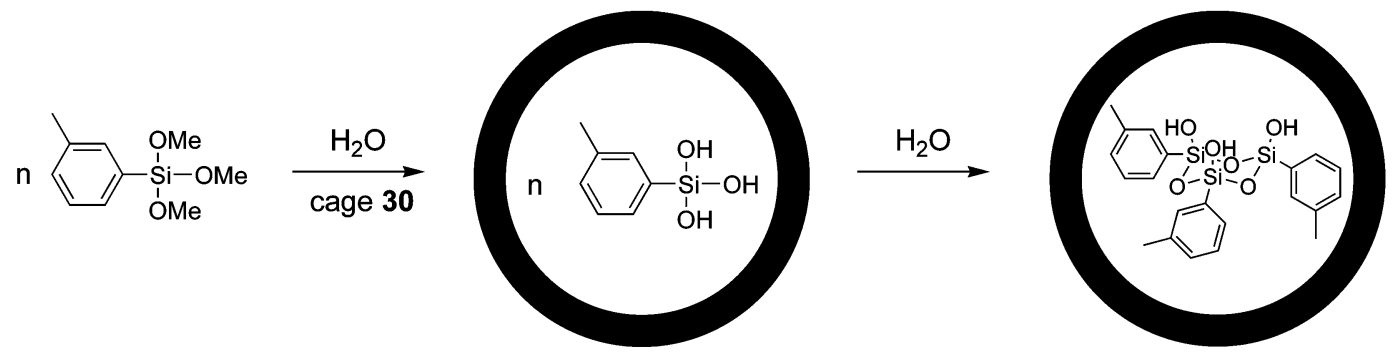

(a)

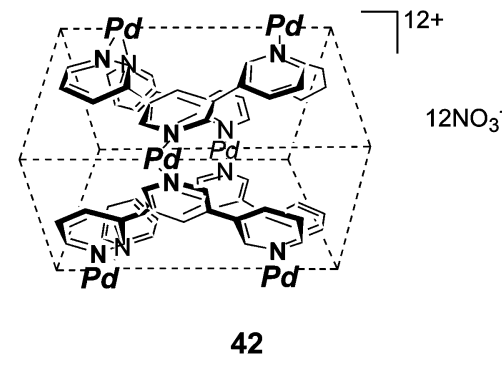

(b)

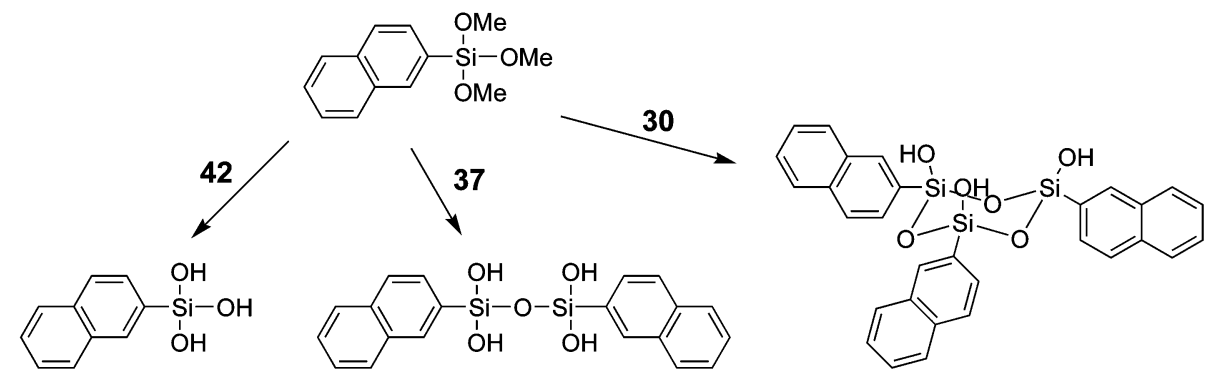

(c)

Figure 9. (a) Stabilization of cyclic siloxane trimers by means of encapsulation by 30. (b) Structure of tubelike cage 42. (c) Cavity-directed synthesis of siloxane oligomers. (Adapted with permission from Chart 1 from ref 102 . Copyright 2002 American Chemical Society.) 
Scheme 3. Encapsulation of Manganese Porphyrin Catalyst 44 within the Self-Assembled Square 43 Consisting of Four Zinc Porphyrins Connected by Four Rhenium Centers
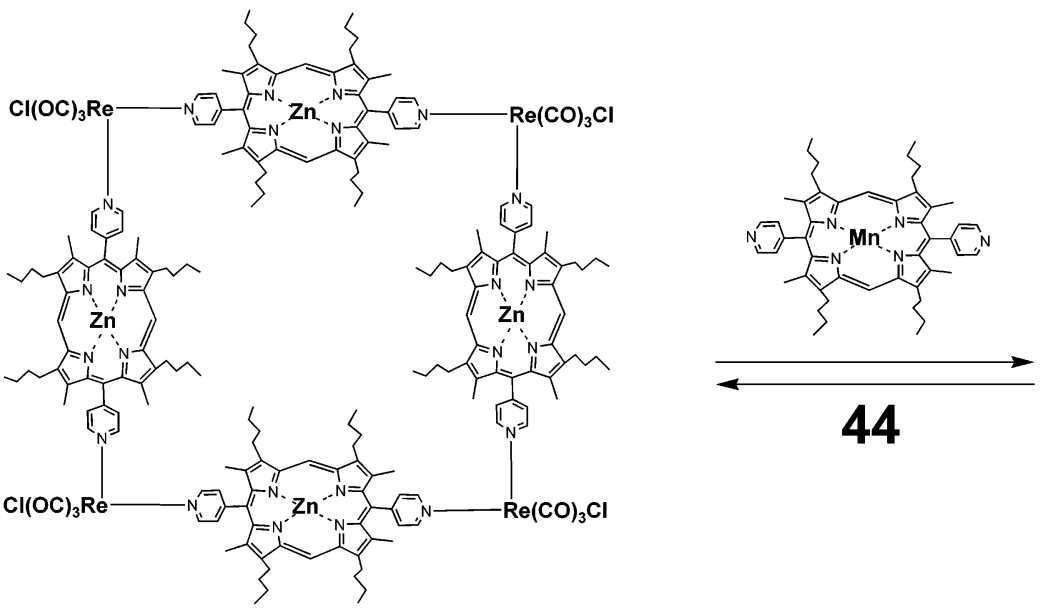

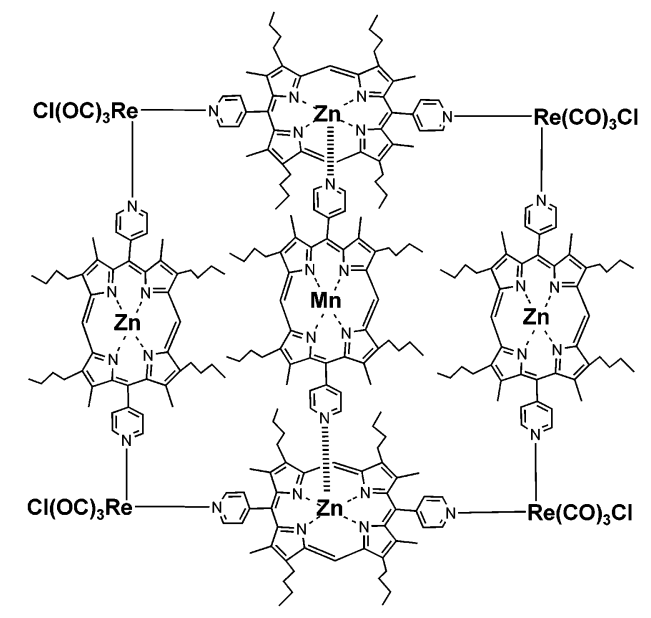

43

stable for more than a week (Figure 9c). Half open cage 37 , which is large enough to accommodate two molecules of substrate, not unexpectedly appeared to be an ideal catalyst for a dimerization reaction in a yield of $88 \%$. Likewise, cage $\mathbf{3 0}$ catalyzed the formation of the cyclic trimer in $90 \%$ yield.

Using a very elegant concept, Nguyen and Hupp have demonstrated the self-assembly and encapsulation of both the catalyst and the substrate within the molecular square $43,{ }^{109}$ which is constructed, in a single step and in quantitative yield, by the coordination of four zinc porphyrins via their pyridyl ligands to four tris(carbonyl)rhenium chloride centers. ${ }^{110}$ The assembly has the shape of an open-ended box with an internal cavity of approximate dimensions $18 \times$ $18 \times 18 \AA$. This preorganized assembly can encapsulate $\mathrm{Mn}$ (III) porphyrin 44, which is a well-known catalyst for the epoxidation of olefins (Scheme 3).

The association constant of this porphyrin within the capsule $\left(K_{\mathrm{a}} \approx 10^{6} \mathrm{M}^{-1}\right)$ ensures a $97 \%$ encapsulation at the concentrations used in dichloromethane solution. This encapsulation protects the catalyst from deactivation, which generally occurs by oxidative degradation through the formation of $\mu$-oxobridged manganese porphyrin dimers. At the same time, the two "half-cavities" present in the complex have a size $(9 \times 18 \AA)$ that is large enough to permit a wide range of olefinic substrates to reach the catalytic center. The encapsulation of the catalyst within the cavity appeared to have great impact on the performance and stability of the catalysts in the epoxidation of styrene. Whereas the nonencapsulated catalysts had a lifetime of about $10 \mathrm{~min}$ and displayed concomitant low turnover numbers $(<500)$, the encapsulated catalyst was stable for many hours and displayed a 10 -fold increase in turnover number. As expected, a tetrapyridyl manganese porphyrin was bound even more strongly in the capsule $\left(K_{\mathrm{a}} \approx 10^{7}\right.$ $\mathrm{M}^{-1}$ ), and, as a result, turnover numbers of 1500 were achieved. Dilution of the catalysts with respect to the capsules resulted in impressive turnover numbers of up to 21000 as a result of enhanced protective encapsulation. In addition, the encapsulated catalysts displayed substrate selectivity in the sense that sterically more encumbered substrates were converted more slowly. The authors claim that the metalloporphyrin catalysts resemble the catalytic core of the enzyme cytochrome P450, while at the same time the encapsulation framework strongly resembles the stability- and selectivity-inducing protein superstructure. The effective cavity size of the system could be further tuned by the coordination of pyridine-derived axial ligands to the zinc ions in the cavities of the capsule, allowing for tailorable substrate size selectivity. More recently, Hupp and coworkers have also applied the empty zinc porphyrin square as a chemosensor for volatile organic guest molecules such as benzene, dioxane, and pyridine. ${ }^{111}$

A novel concept of encapsulating transition metal catalysts was introduced by Reek and co-workers. ${ }^{112}$ The addition of zinc tetrakis (meso-phenyl)porphyrin to $\left[\operatorname{Pd}(\mathbf{4 5})_{4}\right]$ yielded the mono-phosphane palladium complex 46 (Chart 1), which is encapsulated by three zinc porphyrins, whereas the formation of complexes containing more phosphane ligands is prohibited as a result of steric congestion.

This encapsulation-induced metal-ligand reorganization has a dramatic impact on the catalytic

\section{Chart 1}<smiles>c1cncc(P(c2cccnc2)c2cccnc2)c1</smiles>

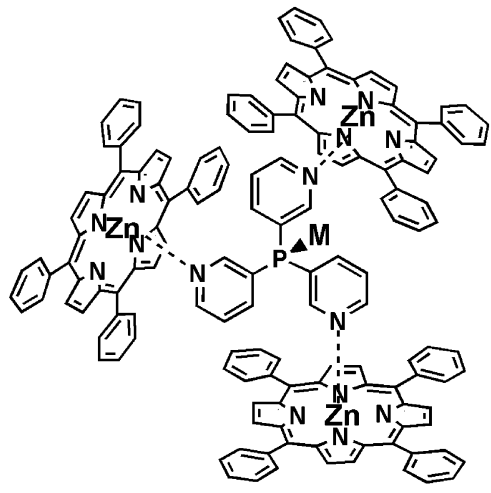

45
$46 \mathrm{M}=\mathrm{PdCl}_{2}$

$47 \mathbf{M}=[\operatorname{Rh}(\mathrm{acac})(\mathrm{CO})]$ 
Encapsulated (unreactive)

Encapsulated (unreactive)

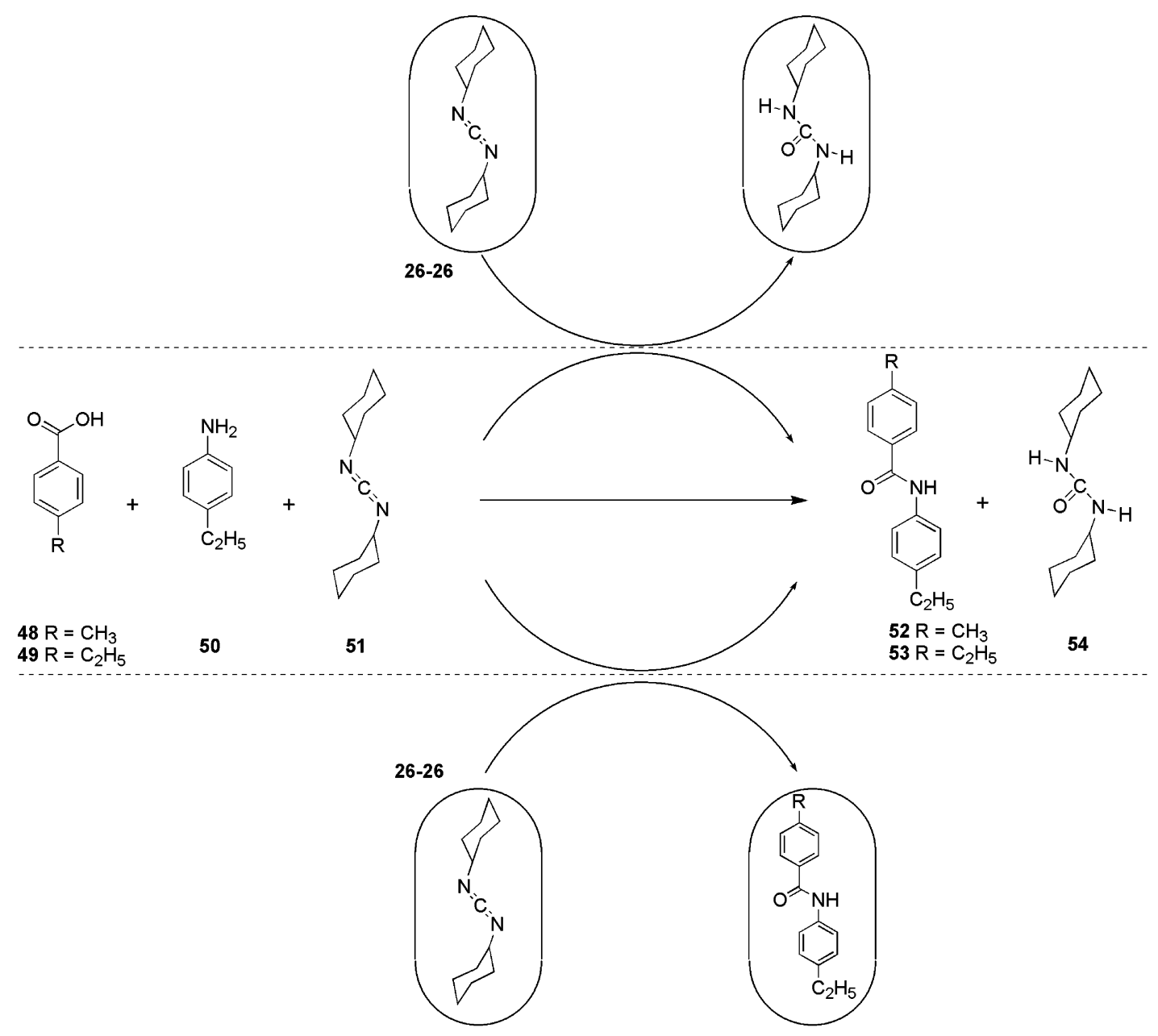

Figure 10. A self-regulating reaction cycle. The release of one molecule of encapsulated DCC results in the formation of two molecules of product (DCU and an anilide), which are capable of displacing two molecules of DCC (see text). (Reproduced with permission from ref 87. Copyright 2002 National Academy of Sciences.)

performance of the palladium center. Whereas $[\mathrm{Pd}$ $(45)_{4}$ ] is inactive as a catalyst in the Heck reaction, the encapsulated complex $\mathbf{4 6}$ undergoes a fast oxidative addition of iodobenzene and it subsequently catalyzes its reaction with styrene. In an analogous fashion, rhodium complex $\mathbf{4 7}$ displayed a 10-fold higher catalytic activity in the hydroformylation of 1-octene as compared to the nonencapsulated [Rh$\left(\right.$ acac) $\left.(\mathrm{CO})(45)_{2}\right]$, and, in addition, the former complex favors the formation of the branched product 2-methylheptanal over the linear 1-octanal, a selectivity that is highly unusual for this substrate. The performance of the encapsulated rhodium catalyst was strongly influenced by variations in the porphyrin metal and substitution pattern, and in the structure of the template ligand. ${ }^{113}$

The examples discussed above all have in common that capsules formed by reversible self-assembly are applied as catalysts in bimolecular reactions, where two (or more) reactive species are bound within a single capsule, as phase-transfer catalysts where the capsules act as shuttles that transport encapsulated guests from one solvent phase to another, or as a confined reaction chambers which stabilize reactive intermediates or convert substrates with enhanced activity or selectivity. In recent years, however, novel approaches have emerged in which capsules are applied in more sophisticated chemical processes. For example, Rebek's group has elegantly demonstrated that encapsulation of reagents can be utilized to effect chemical amplification in a reaction that takes place outside the capsule. ${ }^{87,114}$ The reaction of $p$-toluic acid 48 or $p$-ethylbenzoic acid 49 with $p$-ethylaniline $\mathbf{5 0}$ and $N, N^{\prime}$-dicyclohexylcarbodiimide (DCC) $\mathbf{5 1}$ yields anilides 52 and $\mathbf{5 3}$ at almost identical rates, together with $N, N^{\prime}$-dicyclohexylurea 54 (DCU) and $N$-acylurea side-products (Figure 10). In the presence of a stoichiometric amount of capsule 26-26 (see Figure 4), the reaction rates decreased substantially, and, in addition, $p$-toluic acid appeared to react much faster than $p$-ethylbenzoic acid. Moreover, the addition of anilide $\mathbf{5 2}$ to the reaction mixture accelerated the initial rate of its own formation, whereas the addition of $\mathbf{5 3}$ had no effect. The results are explained by the occurrence of feedback loops in a self-regulating reaction cycle (Figure 10).

DCC is a good guest for the capsule, where it is unreactive, and upon its slow release it can participate in a reaction with an acid and an aniline derivative. In the case of anilide $\mathbf{5 2}$, the product and the DCU side-product are better guests for the capsule than DCC, and overall each single DCC reactant generates two products that effectively displace further DCC molecules. As a result, rate 


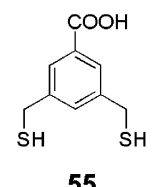

55

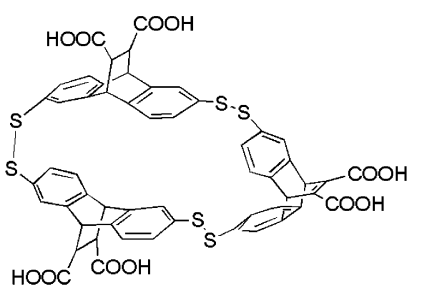

59

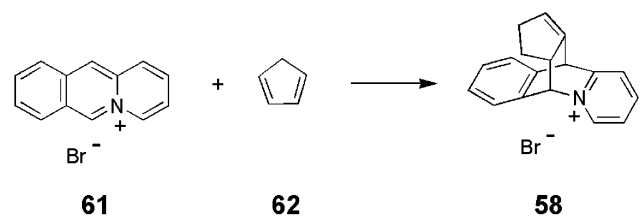

Figure 11. Dynamic combinatorial library components $\mathbf{5 5}-\mathbf{5 7}$, the two products of the library, receptors 59 and $\mathbf{6 0}$, and the synthesis of the template $\mathbf{5 8}$.

acceleration is observed. In contrast, in the case of anilide 53, which does not fit within the capsule, no rate enhancing effect is observed.

A somewhat related example of selection and amplification of a capsular species has been described by Sanders and co-workers. Their approach involves the construction of a receptor by means of a dynamic combinatorial chemistry system, ${ }^{115}$ templated by a guest molecule, after which the same guest can be synthesized using the receptor as a catalyst. ${ }^{116}$ In other words, the templating guest serves as the transition state analogue around which the host is assembled. Although several research groups have been pursuing this approach by making use of molecularly imprinted polymers, ${ }^{117}$ it is difficult to obtain well-defined binding sites whose structures can be altered in a controlled manner. For this reason, research has been directed to solution chemistry in which the bond formation processes in the receptor synthesis are covalent, yet reversible. A library consisting of dithiol building blocks $\mathbf{5 5}, \mathbf{5 6}$, and $\mathbf{5 7}$ was exposed in air to the guest molecule $\mathbf{5 8}$ in slightly basic water (Figure 11).

Under these conditions, dithiol formation by oxidation is sufficiently slow to allow thermodynamic equilibrium to occur. ${ }^{118}$ After a couple of days, two macrocyclic receptors 59 and 60 had been formed, which had both been templated by $\mathbf{5 8}$, constituting $79 \%$ of the library material, whereas they only constituted $12 \%$ of the material in the absence of the template. Because the template $\mathbf{5 8}$ is a product of the Diels-Alder reaction between acridizinium bromide 61 and cyclopentadiene 62 , and the transition state strongly resembles the end product, the newly formed receptors were tested as catalysts in this reaction. Whereas 60 turned out to be catalytically inactive, receptor 59 induced a modest acceleration $(\sim 10$ times) of the Diels-Alder reaction. The inactivity of 60 was attributed to the fact that this receptor complexes the diene starting material more strongly than the product. Surprisingly, only a moderate product inhibition was observed, because the starting compound $\mathbf{6 1}$ is able to compete with $\mathbf{5 8}$ for binding to the catalyst.

\subsection{Micelle-Based Systems}

The cell membrane is probably one of the most distinct examples of how nature uses self-assembly of relatively simple building blocks to create organized structures. ${ }^{119}$ Even the most simple cell is by far superior to man-made reactors with respect to efficiency, yield, etc., and by performing (cascadetype) reactions in such a way that the right reagent is present at the right place at the right time (the coupling of chemical transformations in space and time). The major constituents of the cell membrane are the phospholipids, which are amphiphilic molecules built from a charged phosphate headgroup and a hydrophobic hydrocarbon tail (Table 1). A variety of these amphiphiles have been synthesized, stimulated by the discoveries of Bangham, ${ }^{120}$ that liposomes can be prepared by dispersing isolated phosphatidylcholine molecules in water, and later those of Kunitake, ${ }^{121}$ that vesicles can also be formed by dissolving synthetic didodecyldimethylammonium bromide (DDDAB) in water (for examples of amphiphilic molecules discussed in the following section, see Table 1).

The self-assembly of amphiphiles in water is driven by the hydrophobic effect, ${ }^{122}$ which is probably best described by taking into consideration the solvation thermodynamics that play a role in the favorable overlap of the hydration shells of the hydrophobic parts of the molecules on self-assembly. ${ }^{123}$ As a result, the molecules start to aggregate in aqueous solution above a certain concentration, the so-called critical aggregation concentration (CAC). A variety of aggregate morphologies has been observed, ${ }^{124}$ determined by the combination of the following three terms of free energy:

(i) a favorable contribution resulting from the clustering of the hydrophobic parts of the molecules;

(ii) a surface term reflecting the balance between the tendencies of the amphiphiles to closely pack to minimize unfavorable hydrocarbon-water interactions and to spread apart as a result of the electrostatic repulsion between the (charged) headgroups, hydration effects, and steric hindrance; and

(iii) a packing term, which requires that the hydrophobic cores of the aggregate exclude water and polar headgroups, limiting the possible geometries of the aggregates.

For the aggregation of phospholipids, a simple model has been developed by Israelachvili based on the geometry of the molecules. ${ }^{125,126}$ This model defines a packing parameter $p=v / l a$, in which $a$ is the surface area of the headgroup and $v$ and $l$ are the volume and length of the alkyl chains, respectively. For $0<p<1 / 2$, the formation of micelles is predicted, vesicles are formed when $1 / 2<p<1$, and inverted structures are expected for $p>1$ (Figure 12). 
Table 1. Overview of Amphiphilic Molecules Discussed in Sections 2.2 and 2.3

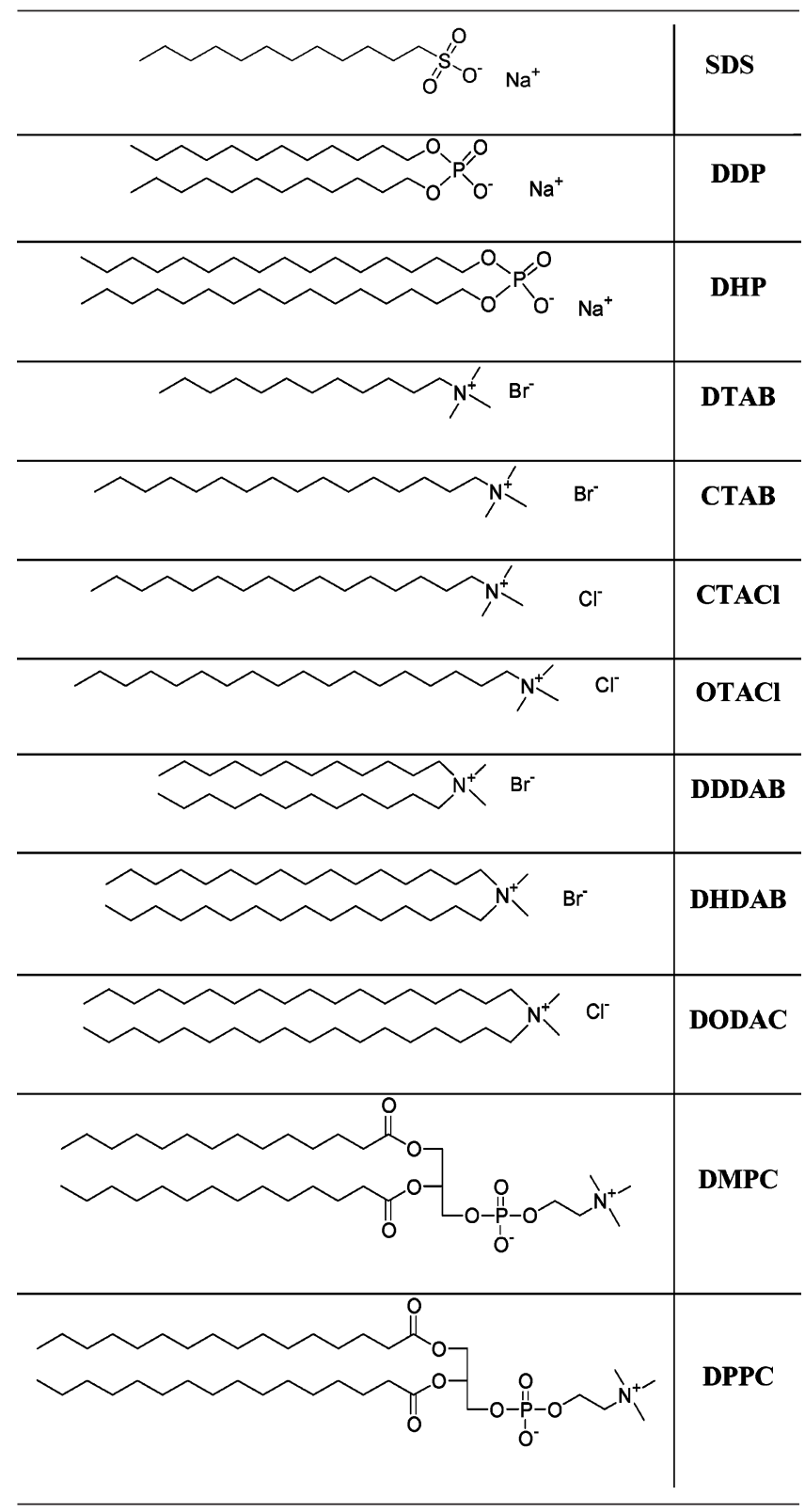

All above aggregate morphologies have dimensions in the nanometer regime. The inclusion of a sort of functional (catalytic) entity inside the self-assembled structure will result in the formation of a (nano)reactor. In this connection, micellar systems are related to microemulsions, that is, microheterogeneous mixtures of oil, water, and surfactant. This is an interesting field of research as microemulsions have been used as reactors, for example, for the preparation of metal particles of finite size. This type of research is, however, beyond the scope of the present review. ${ }^{127}$

As mentioned above, surfactants with $p=<1 / 2$ will form micellar aggregates upon dispersal in water. Mainly depending on the concentration, thermodynamically stable morphologies will be formed; going up in concentration, these are spherical, rodlike, and hexagonal morphologies. Aqueous micelles are kinetically very labile, and the average lifetime is usually in the order of milliseconds, a rate which is

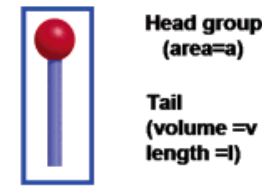

Packing Parameter:
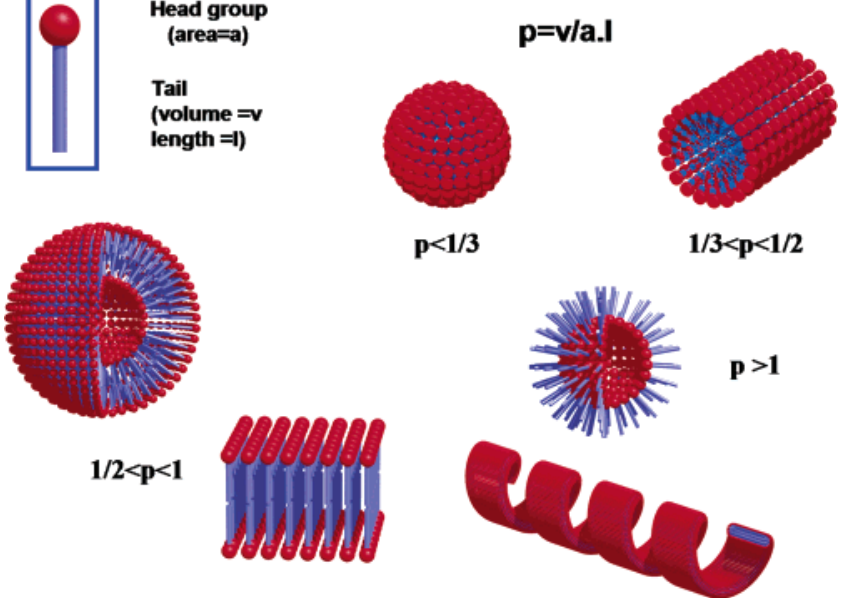

Figure 12. Different aggregate morphologies predicted by the packing parameter $(p)$.

comparable or faster than most chemical reactions. ${ }^{128}$ As a consequence of the difference in polarity between the hydrophilic surface of a micelle and the hydrophobic core, it can solubilize both polar and nonpolar reactants from the aqueous phase and in that way increase or reduce the rate of a reaction. ${ }^{128,129}$ In the case of an enhanced reaction profile, either with respect to the rate or (stereo)selectivity, this effect is referred to as micellar catalysis. This enhancement can be the result of (or a combination of) the following phenomena: (i) an increased local concentration of the reactants at the surface or in the interior of the micelle; (ii) stabilization of the transition state of the reaction due to a favorable interaction with the surfactant molecules; and (iii) a combined polarity, microviscosity, and charge effect inside the micelle (often referred to as a medium effect). ${ }^{130,131}$ Although micelles are not really well-defined systems when compared to vesicles or self-assembled capsules (with respect to both shape and kinetic stability, vide infra), in the above context one might envision them as nanoreactors. For that reason, we thought it appropriate to highlight some examples of micellar catalysis in this overview, focusing on recent studies as this field has been extensively reviewed. ${ }^{128-135}$

Organic conversions in water aided by surfactants have been reported for decades, ${ }^{134}$ but for long this has been limited to relatively simple reactions, for example, the hydrolysis of esters. ${ }^{136}$ Although it should be mentioned that surfactant-mediated emulsion polymerization was already reported in the late 1940s by Harkins, ${ }^{137}$ Menger and co-workers were the first to publish transition metal-catalyzed reactions in water in the presence of surfactants in $1975 .{ }^{138}$ More recently, biomimetic oxidation reactions in aqueous micelles were reported by Rabion et al. ${ }^{139}$ following up on earlier reports on aqueous oxidations using transition metal catalyst in the presence of surfactants. They describe a methane monooxygenase enzyme model consisting of iron complexes, which in the presence of cetyltrimethylammonium hydrogensulfate oxidize cyclohexane with the help of tert-butylhydroperoxide to form cyclohexanol and cyclohexanone. The need of the surfactant was clearly shown by the absence of any catalytic conversion 


\section{Chart 2}

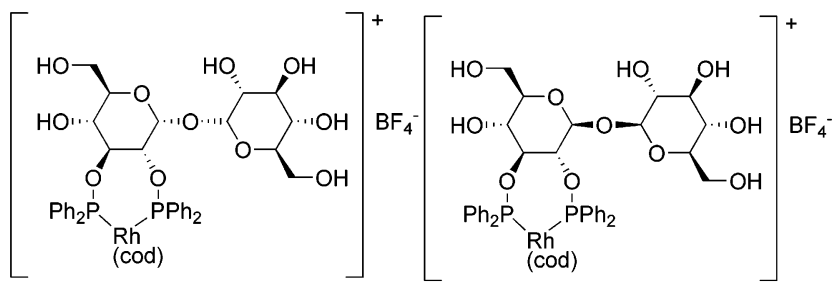

63

64

when the same system was applied under biphasic or emulsion conditions. ${ }^{139}$ Reductive reactions in water catalyzed by transition metal complexes have been shown to be promoted by surfactants as well. ${ }^{140}$ Micelle-forming amphiphiles significantly increase both the reaction rate and the enantioselectivity of $\mathrm{Rh}(\mathrm{I})$-catalyzed hydrogenations of amino acid precursors. ${ }^{141,142}$ This enhancement is only observed when the concentration of the surfactant is above its CMC. ${ }^{143}$ Water-soluble rhodium complexes containing glucose-based phosphino ligands $(\mathbf{6 3}, 64$, Chart 2 ) proved to be effective catalysts in the asymmetric hydrogenation of various enamides and itaconic acid in water in the presence of SDS. The use of SDS significantly improved the enantioselectivity, which was suggested to be a consequence of micelle formation. ${ }^{144}$ Mechanistic studies on this enantioselective process have been conducted by Ludwig et al. ${ }^{145}$ using pulsed field gradient spin-echo NMR experiments. It was found that association of the catalyst to the micelles is of key importance for obtaining the increase in enantioselectivity. The authors, however, point out that it is difficult to extrapolate these findings to transition metal catalysis in micellar systems in general.

An elegant rhodium-based example of micellar catalysis was reported by Fuji et al. ${ }^{146}$ A cascade-like catalytic system was used to carbonylate enynes using formaldehyde as a carbon monoxide donor. In this example, the decarbonylation of formaldehyde by the rhodium catalyst took place in the aqueous bulk phase, while the carbonylation reaction occurred in the SDS micellar nanoreactor (Scheme 4). This Pauson-Khand-type reaction is more efficient in this micellar system, which is potentially beneficial to a variety of carbonylation reactions.

With the intention to develop more environmentally friendly reaction conditions, Kobayashi and co-
Scheme 5

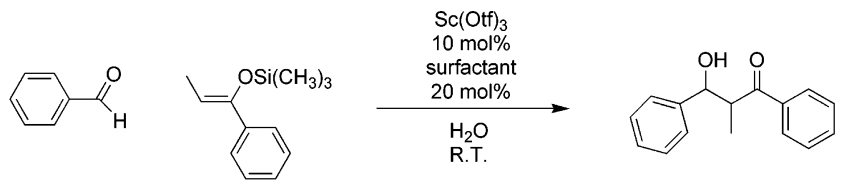

Table 2. Effect of Surfactants on Aldol Reaction in Water $^{147}$

\begin{tabular}{lcc}
\hline surfactant & time $(\mathrm{h})$ & yield $(\%)$ \\
\hline & 4 & 3 \\
SDS & 4 & 88 \\
Triton X-100 & 60 & 89 \\
CTAB & 4 & trace \\
\hline
\end{tabular}

workers studied a variety of acid-catalyzed reactions in water in the presence of surfactants. ${ }^{147}$ In a model reaction, first the surfactant aided Lewis acidcatalyzed aldol reaction depicted in Scheme 5 was tried. ${ }^{148}$ The results of this study are summarized in Table 2. An unexpected enhancement of the reactivity was found when sodium dodecyl sulfate (SDS, $20 \mathrm{~mol}$ $\%, 35 \mathrm{mM})$ was added to the $\mathrm{Sc}(\mathrm{OTf})_{3}$ catalyst. A nonionic surfactant (Triton X-100) was also effective in this aldol reaction, but only a trace amount of product was observed when a cationic surfactant (CTAB) was used. These results prompted the investigators to combine the surfactant with the catalyst. This so-called LASC (Lewis acid-surfactant combined catalyst) was expected to act both as a Lewis acid to activate the reactants and as a surfactant to form stable colloidal dispersions. ${ }^{149}$ These types of catalysts were subsequently successfully used in water to perform several other carbon-carbon bond forming reactions such as allylations and Mannich-type conversions. ${ }^{150}$ Light scattering and microscopy studies on these systems revealed that the colloidal dispersion consisted of micrometer-sized spherical particles, for which it was suggested that the substrates and catalysts are concentrated in the hydrophobic interior, in this way enabling the organic conversions to take place rapidly. These LASC reactors, however, exceed in size the nanometer-regime. Recently, it was shown that in a similar system using $\mathrm{Fe}$ (III) as a Lewis acid catalyst, the aldol reaction of Scheme 5 proceeded in a diastereoselective fashion. ${ }^{151}$

Another important $\mathrm{C}-\mathrm{C}$ bond forming reaction in synthetic organic chemistry is the Diels-Alder (DA) reaction. Many procedures have been developed to

\section{Scheme 4}

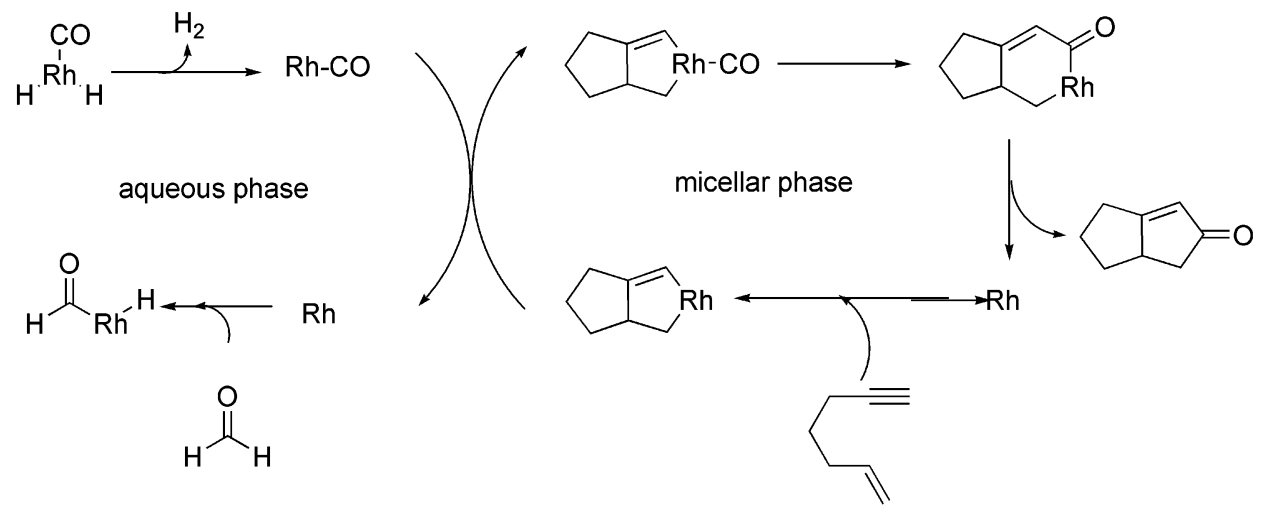




\section{Scheme 6}

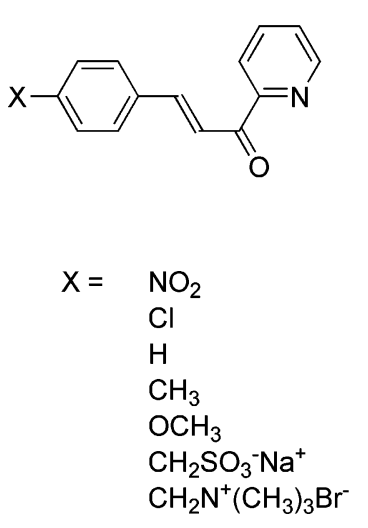

improve the yields and (stereo)selectivity of this type of cycloaddition. Important milestones in these area are the discovery that Lewis acids can catalyze DA reactions in organic solvents, ${ }^{152}$ and that in water remarkable accelerations of the reaction are possible. ${ }^{153}$ The applicability of the DA reaction was extended by Otto and others, ${ }^{154}$ by performing the Lewis acid-catalyzed reaction in micellar media. The reaction depicted in Scheme 6 was initially performed in micelles formed from SDS, CTAB, and $\mathrm{C}_{12} \mathrm{E}_{7}$ (dodecyl heptaoxyethylene ether). In these cases, it was found that the conversions were slowed. The diene and the dienophile apparently reside in different locations of the micelle. In contrast, this reaction turned out to become highly efficient when it was performed in $\mathrm{Cu}(\mathrm{DS})_{2}$ micelles, and rate enhancements up to 1.8 million as compared to the uncatalyzed reaction in acetonitrile were measured. Complete complexation of the dienophile to the copper ions at the micellar surface accounts for this effect. In this way, both reactants are brought in close proximity of each other at the micellar surface, possibly in an even more favorable orientation to give a distinctly more efficient DA reaction. The micelle here indeed acts as a nanoreactor as it brings the reactants together and provides a confined reaction environment. ${ }^{155}$

\subsection{Vesicle-Based Systems}

The importance of vesicles as potential membrane models was first recognized by Gebicki and Hicks. They prepared closed bilayer structures in aqueous solution by shaking thin films of oleic and linoleic acids, however, with poor solubility of the formed aggregates. ${ }^{156}$ In 1977, Kunitake and co-workers ${ }^{121}$ gave the first example of vesicle formation by a completely synthetic amphiphile, viz., DDDAB (Table 1). This opened the way to study in detail the properties of biomembranes and mimic their function, using a variety of (charged) synthetic surfactant molecules.

\subsubsection{Reactions in or at the Vesicle Membrane}

As was exemplified in the previous section (2.2), the surface of an aggregate can have an outspoken effect on reactions taking place in its direct surroundings. A vesicle surface is more organized than
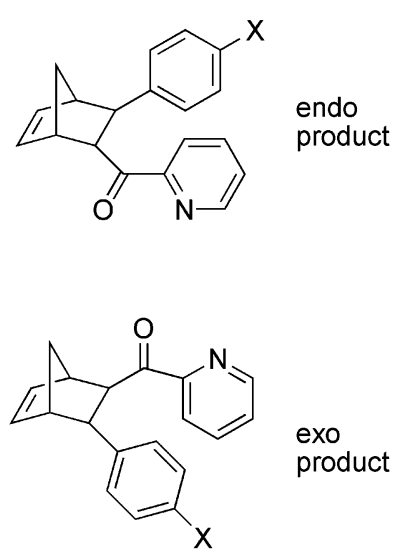

Scheme 7

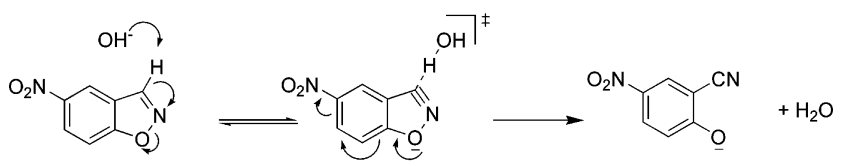

the surface of a micelle and potentially can influence the reaction pathway of conversions better than a micelle surface can do.

The group of Engberts has employed surfactant aggregates as simple enzyme mimics, with the aim of understanding the efficiency of (artificial) enzymes in terms of desolvation effects, entropy factors, etc. For that reason, the Kemp elimination (Scheme 7) was studied in the presence of aggregates formed by a series of surfactants. ${ }^{157-159}$ With respect to the reaction in pure water at the same $\mathrm{pH}$, the elimination rate was increased up to 850 times. It turned out that vesicles were more effective catalysts than micelles, likely providing a more apolar microenvironment at the substrate binding sites for the deprotonation of the substrate. The high overall rate enhancement is expected to be the result of a catalytic reaction involving less strongly hydrated hydroxide ions. ${ }^{157}$ This hypothesis was further confirmed by detailed kinetic studies on the same elimination reaction performed in the presence of positively charged DODAC vesicles in which different amounts of negatively charged DDP (Table 1) were mixed. ${ }^{158}$ The introduction of various other additives, such as linear alcohols and alkyl pyroanosides, led to changes in the vesicular catalysis reflecting its dependence on the molecular properties of the interface between the vesicle and bulk water. ${ }^{159}$

The results obtained by the Engberts' group indicate that a highly complex mix of factors is involved in catalytic reactions occurring in synthetic and probably also in biological cell membranes. One of these factors is the counterion, which not only acts as a charge compensator but also can function as an active catalyst as already exemplified in the case of micelles (vide infra). In bilayers formed by cerium phosphates, for example, it was found that the hydrolysis of DNA can be catalyzed by the counterion. ${ }^{160}$ Rispens and Engberts reported the efficient catalysis of a Diels-Alder reaction by copper(II) vesicles (65, Chart 3) and compared the maximum rates of the reaction with a similar micellar system 
Chart 3

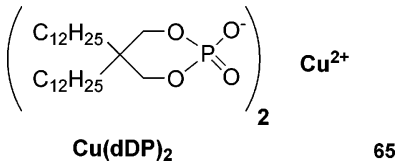

and with the uncatalyzed reaction in acetonitrile. It was found that the rate increased by $1.5-2$-fold when going from the micellar to the vesicular system, but the rate enhancement as compared to the uncatalyzed reaction was million fold. Furthermore, it was observed that catalysis already occurred at 10-20 times lower concentration for the metallo-vesicles than for the metallo-micelles. More efficient binding of the substrate to the small vesicles possibly explains the observed differences. ${ }^{161}$ In these examples, the counterion is the active catalyst. In the following part of this section, preformed (transition) metal complexes anchored to vesicular nanoreactors act in this way.

Cytochrome P450 is an oxidative enzyme involved in a diversity of natural processes; ${ }^{162,163}$ next to oxidations it is also active in dehydrogenation reactions, oxidative formylations, dehydrations, etc. ${ }^{164}$ Cytochrome P450 is a membrane-bound enzyme, a feature which prompted numerous research groups to design model systems in which metal porphyrins (a synthetic equivalent of the iron(III) protoporphyrin IX present in the active center of the enzyme) are incorporated in the bilayers of vesicle membranes. ${ }^{165}$ The first studies in this direction were conducted by Sorokin et al. in the early 1980 s. ${ }^{166}$ Oxidation reactions catalyzed by the relatively simple complex manganese(III) tetrahexadecylphenyl porphyrin chloride incorporated in the membrane of DMPC (Table
1) vesicles were studied using iodosobenzene as an oxidant. In a comparable system built from DMPC or DPPC (Table 1) vesicles, regioselectivity was obtained in the epoxidation of steroids and polyunsaturated fatty acids by employing a membrane spanning iron(III) tetrakis(o-cholenylamidophenyl)porphyrin (Fe(III)ChPP) as catalyst. ${ }^{167}$ The same porphyrin, with a different metal in its central core (i.e., $\mathrm{Mn}(\mathrm{III}) \mathrm{ChPP}$ ), was used to construct a selfassembled system, which by reductive activation of molecular oxygen produced $20 \mathrm{~mol}$ of acetophenone per mol of Mn from ethylbenzene. The presence in the membrane of the enzyme flavoprotein pyruvate oxidase was essential for the functioning of this hybrid enzyme-synthetic catalyst. From the oxidative decarboxylation of pyruvic acid, this enzyme obtains electrons that are carried to the porphyrin by an amphiphilic flavin (Figure 13).

The group of Nolte has studied a P450 mimic, which involved a vesicular nanoreactor that was stabilized by polymerized isocyanide functions present in the DHDAB amphiphiles forming the vesicle bilayer. All features of the natural enzymatic system were included in this nanostructure, that is, a metalloporphyrin with an axial ligand as catalyst, methylene blue as the electron carrier, molecular oxygen as oxidant, and finally $\mathrm{H}_{2} /$ colloidal $\mathrm{Pt}$ as the electron donor. During the vesicle preparation step, the porphyrin was incorporated in the membrane and the colloidal platinum in the aqueous interior. This catalytic system was able to epoxidize both watersoluble (2,5-dihydrofuran) and water-insoluble (styrene) alkenes under an atmosphere of oxygen and hydrogen gases (1:1). ${ }^{168}$

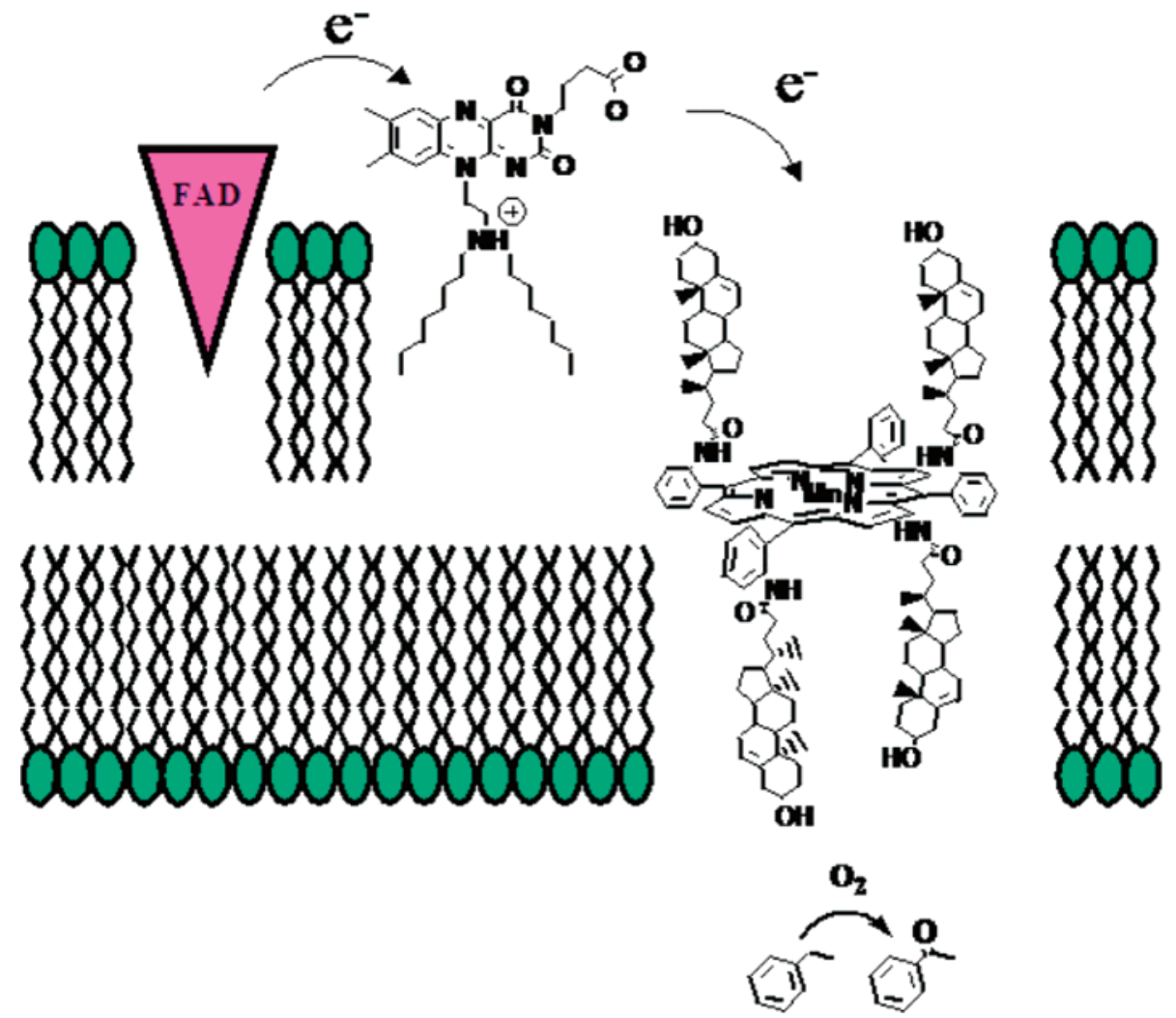

Figure 13. Epoxidation of ethylbenzene in a vesicle nanoreactor by a mixed enzyme-synthetic catalyst. 


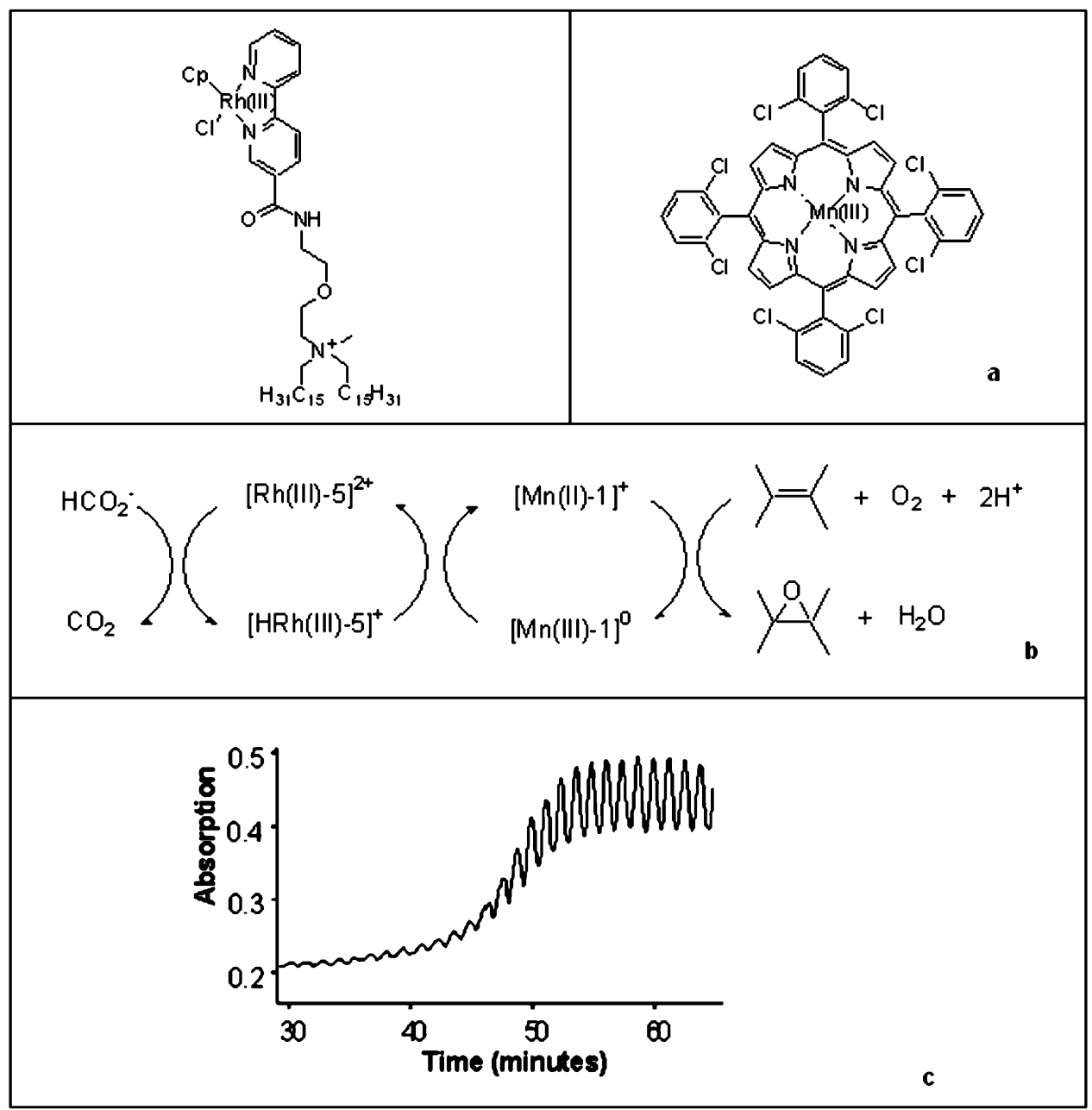

Figure 14. Rhodium complex and manganese porphyrin (a), redox cycle (b), and oscillating behavior as measured at $\lambda=$ $435 \mathrm{~nm}$ in the UV/vis spectrum (c) of Nolte's P450 mimic. ${ }^{169-171}$ (Adapted with permission from Figure 2 from ref 171. Copyright 1995 American Chemical Society.)

The poor efficiency of the $\mathrm{H}_{2} /$ colloidal Pt system as the electron-donor urged this group to find an alternative reducing agent for molecular oxygen, viz., a $\mathrm{Rh}(\mathrm{III}) /$ formate system. In DHP and DODAC vesicles, manganese tetradichlorephenylporphyrin acetate (MnTDCPP acetate), an amphiphilic rhodium complex, and $N$-methylimidazole (Figure 14) were introduced. ${ }^{169}$ Detailed mechanistic studies revealed that the reduction of the $\mathrm{Rh}$ (III) is fastest in DODAC vesicles, likely the result of an increased concentration of the negatively charged formate ions at the positively charged vesicle surface. Consequently, also the reduction of the mangenese porphyrin incorporated in the vesicle bilayer was the fastest in DODAC vesicles. In the aimed epoxidation reaction, however, no conversion of the substrate (styrene) was observed using the positively charged DODAC vesicles, whereas with the DHP vesicles a turnover of $60 \mathrm{~mol}$ of substrate per mol of porphyrin per hour at $70^{\circ} \mathrm{C}$ was found. This was explained by the fact that the concentration of protons in the DODAC systems was too low to allow the formation of the catalytically active $\mathrm{Mn}(\mathrm{V})$ oxo species. ${ }^{170}$

Under very specific conditions (temperature, concentration of reagents), the enzyme mimetic system displayed an unprecedented oscillatory behavior. Under the chosen circumstances and at a precise ratio of $[\mathrm{Rh}] /[\mathrm{Mn}]=10$ after an induction period of $30 \mathrm{~min}$, the $\mathrm{Mn}$ (II) species was formed, which was oxidized back to the $\mathrm{Mn}$ (III) by the $\mathrm{O}_{2}$ present in the medium. After approximately $50 \mathrm{~min}$, the reaction started to oscillate between the $\mathrm{Mn}$ (III) and $\mathrm{Mn}$ (II) oxidation states (Figure 14), a process that showed to be very sensitive to small changes in temperature and $[\mathrm{Rh}] /[\mathrm{Mn}]$ ratio. It was postulated based on UV/ vis studies, that this shuttling between the $\mathrm{Mn}$ (II) and the Mn(III) states of the catalyst is coupled with a movement of the manganese porphyrin upon reduction to a less polar part of the bilayer, that is, away from the vesicle surface. ${ }^{171}$

Hemocyanin is an oxygen carrier that forms tubelike aggregates in the presence of $\mathrm{Mg}^{2+}$ ions. The aggregated form of the protein binds molecular oxygen, which coordinates to two copper centers in the active site of the hemocyanin. Binding and activation of oxygen inspired by hemocyanin was one of the objectives in the studies on the aggregation behavior of amphiphile 66 (Figure 15). ${ }^{172}$ This amphiphile has independent binding sites for the coordination of copper ions (i.e., the nitrogen ligands) and alkali metal salts (i.e., the crown ether part). The copper complex $[\mathrm{Cu}(\mathrm{II})(\mathbf{6 6})]\left(\mathrm{ClO}_{4}\right)_{2}$ was found to assemble into different aggregate morphologies, and in the presence of alkali metal salts vesicles were 

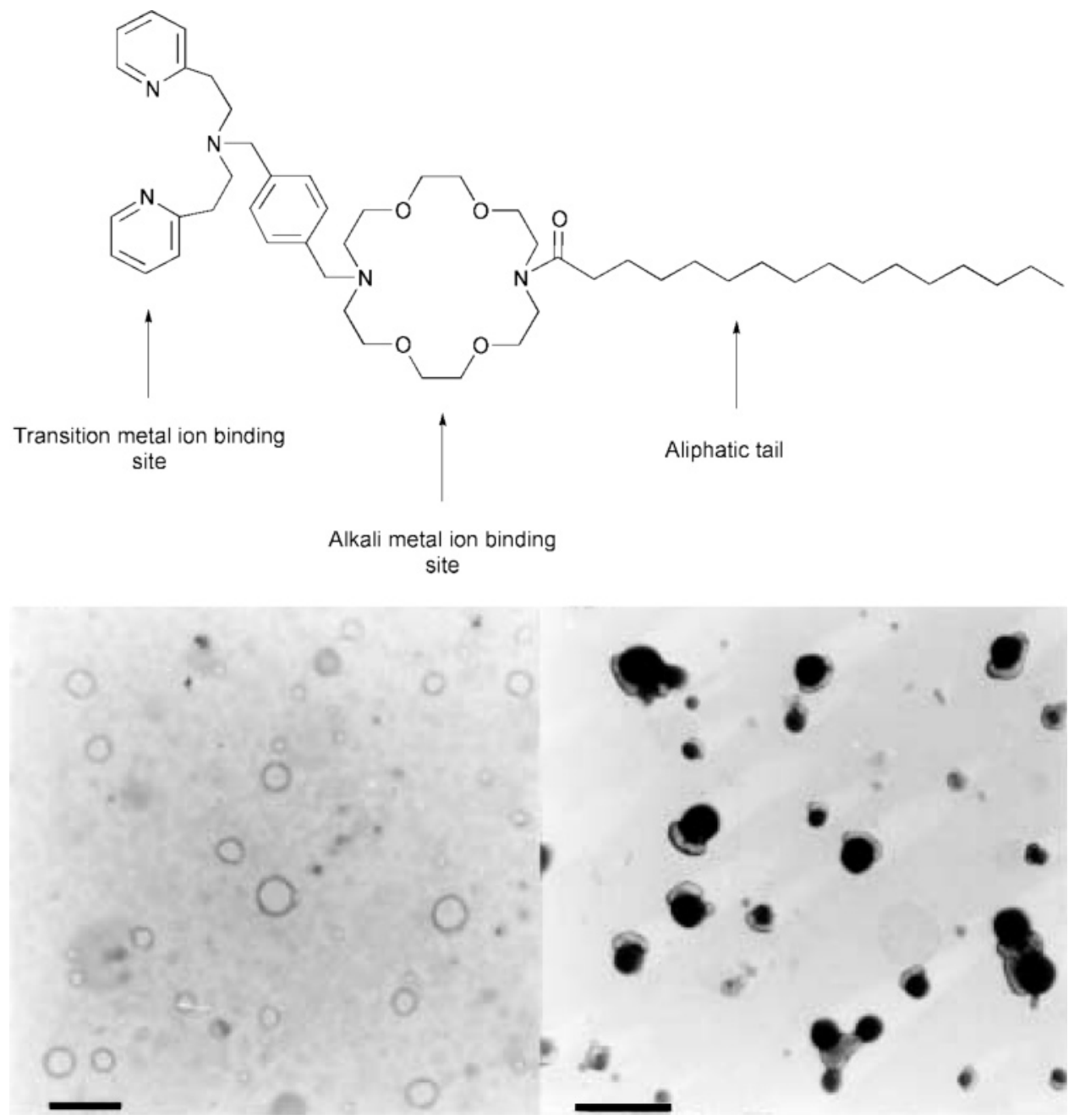

Figure 15. Copper complexing amphiphile 66 (top) and TEM images of vesicles formed from the copper complex of this molecule in the presence of alkali salts $\left(\mathrm{RbClO}_{4}\right)$. Negatively stained (bottom left) and Pt shadowed (bottom right). (Reproduced with permission from ref 172. Copyright 2001 Royal Society of Chemistry.)

formed (Figure 15). UV/vis studies indicated that $\mathrm{O}_{2}$ did not react with the in situ prepared aggregates of $[\mathrm{Cu}(\mathrm{I})(66)]\left(\mathrm{ClO}_{4}\right)_{2}$. In the presence of $\mathrm{O}_{2}$, however, hydroperoxo copper complexes were formed by the aggregates of the copper(II) amphiphile. The complexes were reactive and catalyzed the oxidative degradation of the ligand system $66 .{ }^{172}$

Van Leeuwen and co-workers have studied the aggregation behavior of a series of amphiphilic diphosphine ligands as part of a project to obtain an easy separation of catalyst from product and to increase product conversion in the aqueous rhodiumcatalyzed hydroformylation of 1-octene (Figure 16). ${ }^{173}$ Ligands and their rhodium complexes with a sufficiently large hydrophobic tail formed highly stable vesicles in water, which could be visualized by electron microscopy (Figure 16). The formation of the vesicular aggregates led to an increased solubility of organic substrates in the aqueous solution containing the amphiphilic complexes, which resulted in a higher hydroformylation reaction rate. Consecutive catalytic runs indicated that the nanoreactors stayed intact during recycling and that the rhodium complexes remained active; in all runs more or less similar turnover numbers $(6-8 \mathrm{~mol}$ of product $/ \mathrm{mol}$ of $\mathrm{Rh}^{*} \mathrm{~h}$ ) were found, and also the product selectivity toward the aldehyde was approximately the same $(\sim 97 \%) .{ }^{173}$

The interaction between a cell membrane and a membrane-bound enzyme is even more complex than the interaction of synthetic catalysts with micelles or vesicles. It is well known that phospholipids are a prerequisite for a membrane-bound enzyme to function properly. ${ }^{174}$ Studying enzymes in synthetic membrane systems could be a useful approach to study the biological and biophysical principles underlying the working mechanisms of membrane-bound enzymes, but also practical applications can be foreseen. Examples have been given by Sada and others. ${ }^{175}$ The yield of prostaglandins (physicologically active unsaturated carboxylic acids) from arachidonic acid was almost doubled by incorporating prostaglandin-synthase in a phospholipid vesicle membrane. The interaction between membrane-bound sarcosine dehydrogenase and the surrounding lipids was studied in detail by Kheirolomoom et al. ${ }^{176}$ It was found that the activity of the enzyme increased 25 -fold by reconstituting the enzyme in DMPC vesicles. This was attributed to the induction of favorable conformational changes in both the substrate-binding site and the catalytic site of the enzyme as a result of lipid-protein interactions. The relation between the 

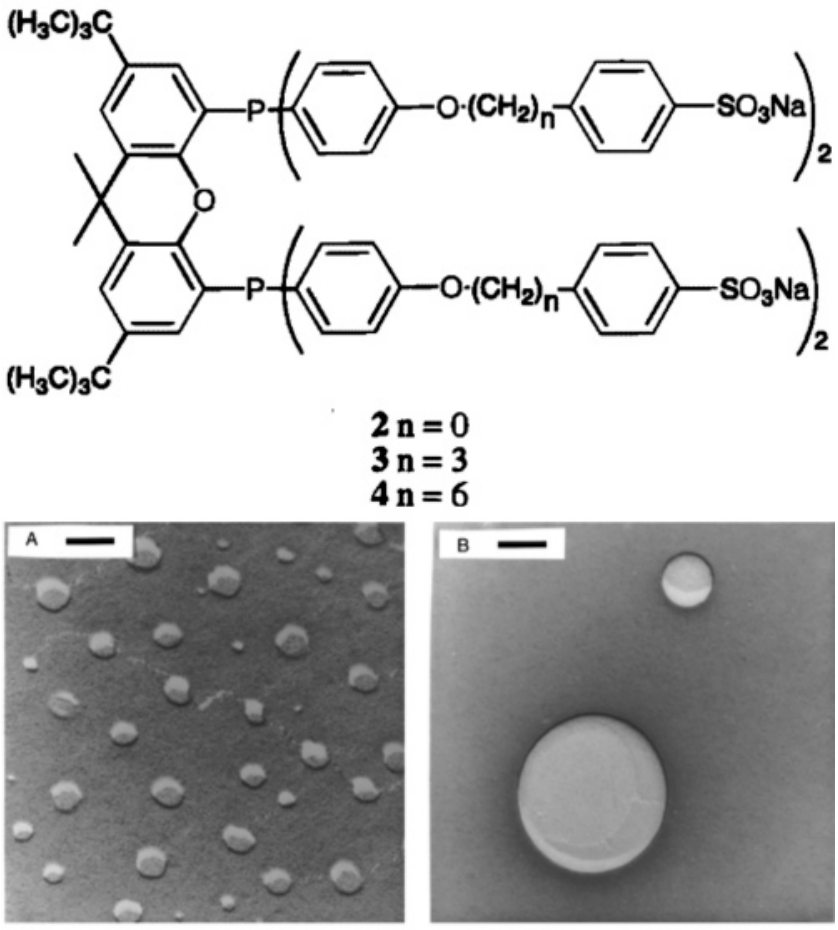

$\mathbf{n}=0$

$3 \mathrm{n}=3$

$4 n=6$
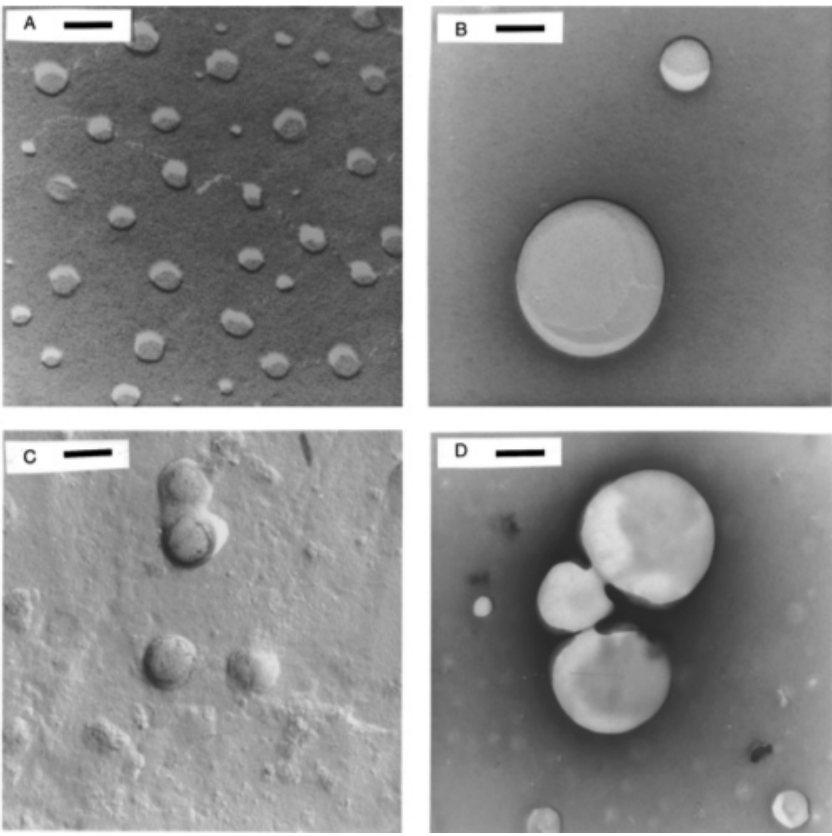

Figure 16. Amphiphilic diphosphine ligands studied by van Leeuwen and co-workers and TEM images of vesicles formed from these amphiphiles. A uniform distribution of vesicles of 3; platinum shadowing technique (a). A large and an average sized vesicle formed by 4 (b). Freezefractured sample of a solution of $\mathbf{3}$ showing the formation of monolayered vesicles (c). Larger aggregates observed shortly after sonication of a solution of the complex between ligand 3 and $\mathrm{RhH}(\mathrm{CO}) \mathrm{PPh}_{3}$ and 1-octene, platinum shadowing technique (d). (Bar size $200 \mathrm{~nm}$.) (Reproduced with permission from ref 173. Copyright 2000 American Chemical Society.)

state (gel or liquid crystalline) of the phospholipid membrane and the activity of the enzyme was furthermore confirmed by the observation of a break in the Arrhenius activation energy close to the gelliquid crystalline transition point of the membrane.

\subsubsection{Reactions in the Inner Compartment of Vesicles}

Soon after Bangham's discovery in the early 1960s that isolated phosphatidylchloline self-organizes into vesicular structures upon dispersal in water, ${ }^{120}$ these aggregates have been envisioned as drug-delivery vehicles (for example, for gene therapy or enzyme replacement therapy) and as bioreactors. ${ }^{177}$ The latter application is of interest within the context of this review, as vesicle aggregates may serve as submicrometer compartments in which enzymatic reactions can take place. ${ }^{178}$ The enzymes are entrapped in the inner vesicle compartment, while the substrate molecules are present in the bulk medium and hence have to cross the vesicle membrane to be converted by the biocatalyst. A variety of potential applications have been foreseen for these vesicle-based nanoreactors, of which their potential to form a kind of artificial cell is one of the most fascinating ones, but this is still a far reached prospect. ${ }^{179,180}$

The set of requirements needed for a liposome to act as a functional micro- or nanometer-sized bioreactor have been listed by Monnard in a topical review. ${ }^{177}$ In summary, these requirements are: (i) the catalytic species should be efficiently entrapped in the vesicle inner compartment; (ii) the reactor membrane must be sufficiently stable and robust to retain the catalytic species and other components and protect them from degradation; the membrane should also allow for (selective) diffusion of the substrate and product molecules; (iii) external influences, such as $\mathrm{pH}$, temperature, and ionic strength, should not destabilize the system to such an extend that it stops functioning, and (iv) the system must be innocuous to allow its use in living systems. In the following section, we will discuss examples of surfactant based bioreactors, or studies toward such reactors, which at least meet the requirements (i) and (ii). They vary from single enzymes in a liposome, ${ }^{178}$ to complex biochemical constructs that are capable of DNA amplification by the polymerase chain reaction (PCR). ${ }^{181}$

Several routes to encapsulate functional compounds in vesicles can be envisaged. They range from straightforward mixing of the amphiphiles with the aimed catalyst followed by a separation step, via extrusion and microfluidization procedures to complex repetative freeze/thaw methods. The physical properties of the vesicles, however, vary greatly with the different preparation procedures, even after homogenation steps. An excellent overview of the utilized procedures has been given by Walde and Ichikawa. ${ }^{178} \mathrm{~A}$ dehydration/rehydration procedure appears to be the best compromise between efficiency and preservation of the catalytic activity. ${ }^{177}$ In this procedure, a mixture of all of the reactors ingredients (empty vesicles, enzymes, etc.) is simultaneously dehydrated. This yields stacked lipid bilayers in which the dissolved (macro)molecules are intercalated. Upon rehydration, the vesicles reform and encapsulate the solutes, resulting in the aimed reactor configuration. It should be noted that during the drying step the lipid bilayers preserve the activity of the enzymes, which otherwise would be lost.

The permeability of the lipid bilayer is also of utmost importance, as the catalyzed reaction in the reactor depends on the diffusion of substrates and products from or to the bulk water phase. This is a complex issue as it depends on both the characteristics of the solute and the amphiphiles building up the bilayers. ${ }^{174}$ In general, two processes can be recognized for spontaneous transport across the membrane. Molecules that are relatively hydrophobic will partly dissolve in the bilayer and in that way diffuse across it. Ionic solutes likely use defects in the bilayer to cross the permeability barrier. ${ }^{177}$ To 
influence these parameters, the properties of the bilayer can be tuned by mixing different amphiphiles or by introducing additives (such as a cholesterol). It is also possible to accomplish active transport across the vesicle membrane, for example, by introducing ion-channels (i.e., transmembrane proteins).

To obtain insight in the processes important for the origin of life, Luigi and co-workers have studied a number of enzymatic reactions inside liposomes. ${ }^{182}$ The first attempt to create a cell model made use of 1-palmitoyl-2-oleoyl-sn-glycero-3-phosphocholine (POPC) vesicles and was aimed at incorporating an enzymatic chain that would convert glycerol-3phosphate into lecithin. ${ }^{183}$ Subsequently, using chemically more simple surfactants, the polymerization of ADP (adenosine 5'-diphosphate) by polynucleotide phosphorylase to yield poly(A) (polyadenosine) was studied. The amphiphiles building up the aggregates (i.e., oleic acid/oleate) displayed interesting selfreproducing properties. The hydrolysis of waterinsoluble precursors of these amphiphiles proceeded in an autocatalytic fashion when the reaction was performed in the presence of both micelles, ${ }^{184}$ and vesicles, ${ }^{185}$ formed by these amphiphiles. It was shown that in a single system both the reproduction of the vesicle and polymerization of ADP took place. ${ }^{186}$ In a different vesicular system, similar ADP polymerization results were obtained by Chakrabarti and co-workers, ${ }^{187}$ and more recently also by Treyer et al. ${ }^{188}$ Using the same technology of encapsulating enzymes into vesicles, biomolecules with varying complexity going from glycogen, ${ }^{189}$ to $\mathrm{RNA}, 190$ and DNA amplification by PCR, ${ }^{181}$ to eventually complete proteins (Green Fluorescent Protein (GFP)182,191), have been constructed. In particular, the latter example of GFP synthesis highlights the propensity of liposomes as reactors. Yu et al. have encapsulated the T7 RNA polymerase system (7 components) and the functional ribosomal machinery (i.e., S30 extract containing 2 ribosomal subunits, elongation factors, 20 amino acids, their tRNA and their aminoacyl tRNA synthetases) successfully into vesicles. Although only a limited amount of the reactors contained all necessary components for the coupled systems, fluorescence was detected from GFP in the internal aqueous core. ${ }^{191}$

Coupled enzyme reactions have been performed in vesicles to release anti-bacterial species. Vesicles have been prepared mainly from DPPC, and in their inner compartment mixtures of glucose oxidase (GOx) and horseradish peroxidase (HRP) were encapsulated. Upon addition of glucose as the primary substrate together with iodide, oxyacids (e.g., hypoiodous (HIO), iodic $\left(\mathrm{HIO}_{3}\right)$, and periodic $\left(\mathrm{HIO}_{4}\right)$ acid) were formed which have anti-bacterial properties (Scheme 8). ${ }^{192}$ The co-encapsulation of both enzymes in the same reactor had a beneficial effect on the conversion of hydrogen peroxide. ${ }^{193}$ This concept could be applied to other peroxidases (Scheme 8), yielding different oxyacids with different antibacterial properties. ${ }^{194}$ The single encapsulation of GOx has been studied in detail, not only as reactor but also as a glucose biosensor. ${ }^{195}$

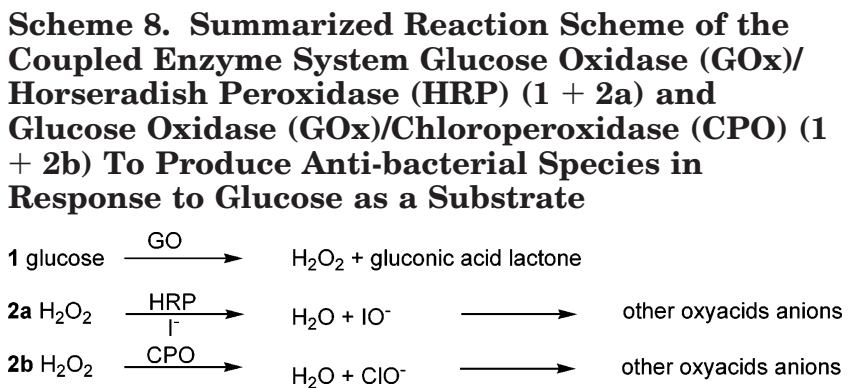

The idea of incorporating catalytically active species in the aqueous compartment of a liposome can also be applied to larger membrane structures, that is, giant vesicles. ${ }^{196,197}$ The size of the giant vesicles (they have 100-1000 times larger diameters than conventional liposomes) gives them some significant advantages. They are formed by electroformation and can be monitored in real time by light microscopy; they can be manipulated in several ways (e.g., punctured and moved), and compounds can be injected into their aqueous inner compartment using a micropipet. In particular, the possibility of injection is very useful when these architectures are used as reactors, because one is entirely sure that all components needed for a chemical reaction are present in the same compartment. Several (biorelevant) processes have been performed in giant vesicles as reactors. ${ }^{198}$ Recently, also self-reproducing giant vesicles have been reported by Sugawara and coworkers (Figure 17). ${ }^{199}$ The membrane of these aggregates was built from amphiphiles that had an imine group in their hydrophobic part, which is the product of a condensation reaction between an amphiphilic aldehyde and a lipophilic aniline derivative. Addition of the aldehyde precursor to preformed giant vesicles, which contained the aniline precursor and a catalyst, resulted in the formation of new amphiphiles inside the vesicular compartment. Smaller vesicles were subsequently formed in this inner water pool, and in a kind of exocytosis process these vesicles were excreted from the parent giant vesicle.

The fabrication of nanosized reactors by selfassembling amphiphiles into micellar or vesicular architectures has been shown to influence a variety of reactions. This ranges from simple ester hydrolyses aided by micelles to the total synthesis of a protein by (part of) the biological machinery encapsulated in the interior of a vesicle. In particular, this latter example is an outspoken illustration of the envisioned self-assembled nanoreactor. It combines multiple components of a synthetic pathway in a confined environment, and, by allowing transport of reagents across the bilayer membrane, this system provides a first step toward controlling reactions in space and time.

Vesicular nanoreactors have been prepared in multiple ways; sometimes just mixing the different components gives a working reactor, but more often several different techniques have to be applied to get the optimal performance. The preparation of bioreactors seemed most efficient using the dehydration/ rehydration method, possibly in combination with extrusion. From the above overview, it can be concluded that working nanoreactors have been pre- 

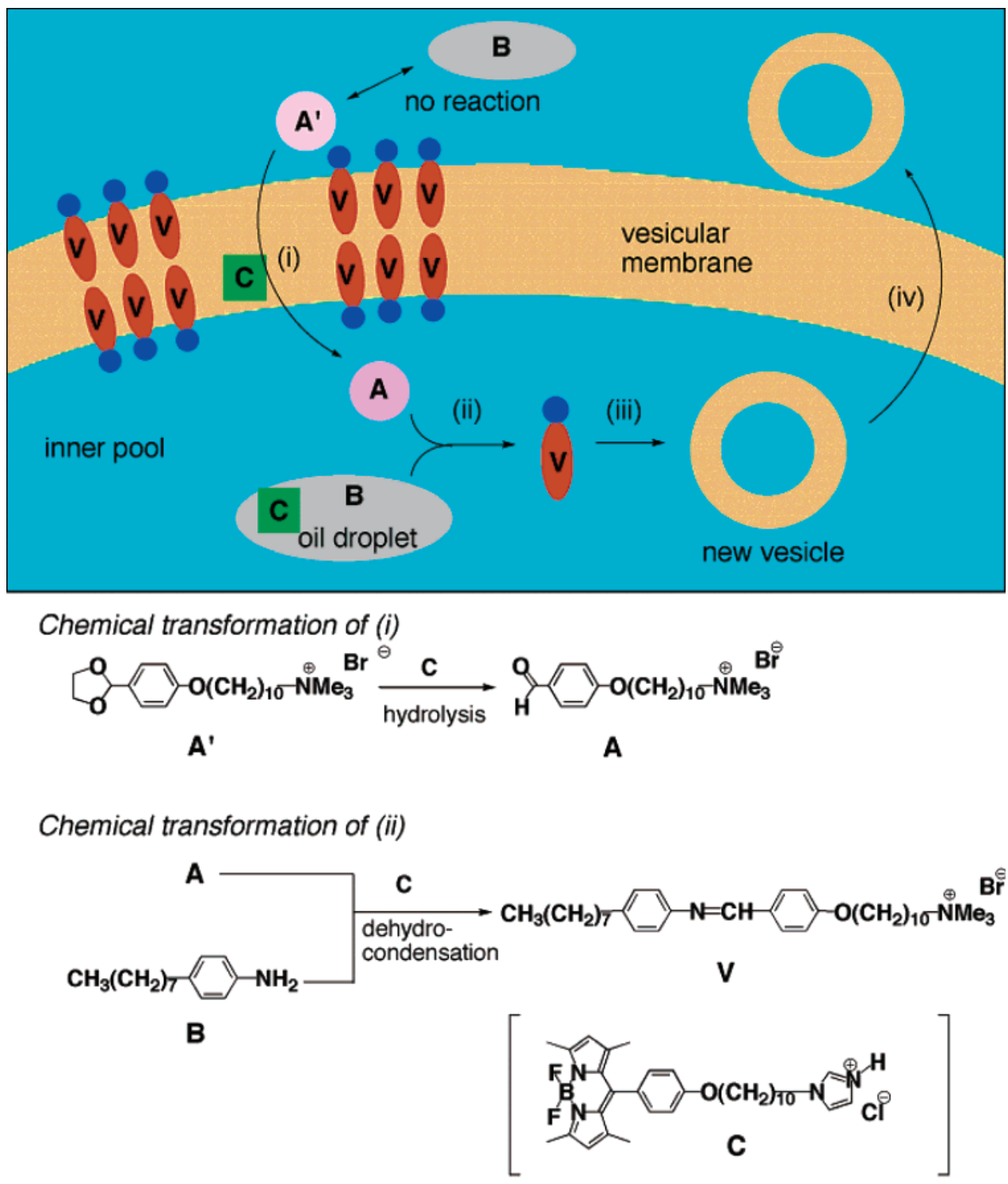

Figure 17. Self-reproducing giant vesicles: (i) locked precursor $\mathbf{A}^{\prime}$ is incorporated into a vesicle composed of $\mathbf{V}$ and catalyst $\mathbf{C}$ and is unlocked to produce reactive precursor $\mathbf{A}$; (ii) $\mathbf{A}$ reacts with the lipophilic precursor $\mathbf{B}$ inside the vesicle to form vesicular molecule V; (iii) new vesicles are generated as $\mathbf{V}$ is produced; (iv) generated vesicles are extruded through the membrane to the bulk water. (Reproduced with permission from ref 199. Copyright 2003 American Chemical Society.)

pared, which are able to perform more and more complex reactions. Some questions, however, remain to be answered. ${ }^{178}$ Where are the enzymes located (inside the water pool or absorbed to the membrane)? Are all enzymes still active after the encapsulation procedure? Is there any leakage of the catalysts? To answer these questions and to explore the potential applications of these types of nanoreactors (e.g., in anti-bacterial films, cascade reactions, cell mimics, etc.), numerous research groups continue to work in this field, and new and exciting developments are on the horizon.

\section{Macromolecular Nanoreactors}

In this section, we will focus on polymers as building blocks for micro- and nanoreactors. Polymers exist in a wide variety of compositions, molecular weights, dispersities, functionalities, shapes, etc. Nowadays, they can be tailor-made from a large choice of monomers, allowing the fine-tuning of their properties. This makes them attractive compounds for numerous applications, among others in industry. ${ }^{200-202}$ The application of polymers as microor nanoreactors, either as single macromolecules possessing hollow interiors, or as self-assembled structures having one or more cavities, is a new, emerging field. Amphiphilic block copolymers, constructed from at least two blocks with different properties, tend to aggregate in solvents selective for one of the constituent blocks, thus resembling traditional surfactants. Analogous to surfactants, a range of morphologies can be observed when dispersions of amphiphilic copolymers are prepared, that is, micellar, ${ }^{203}$ bilayer, ${ }^{204}$ chiral, ${ }^{205}$ and other architectures $^{206}$ (Figure 18). The driving force for the selfassembly is generally considered to be microphase separation of the insoluble blocks. ${ }^{203}$

\subsection{Polymersomes as Nanoreactors}

Vesicles can be prepared from macromolecular amphiphiles, that is, block copolymers, and they are 

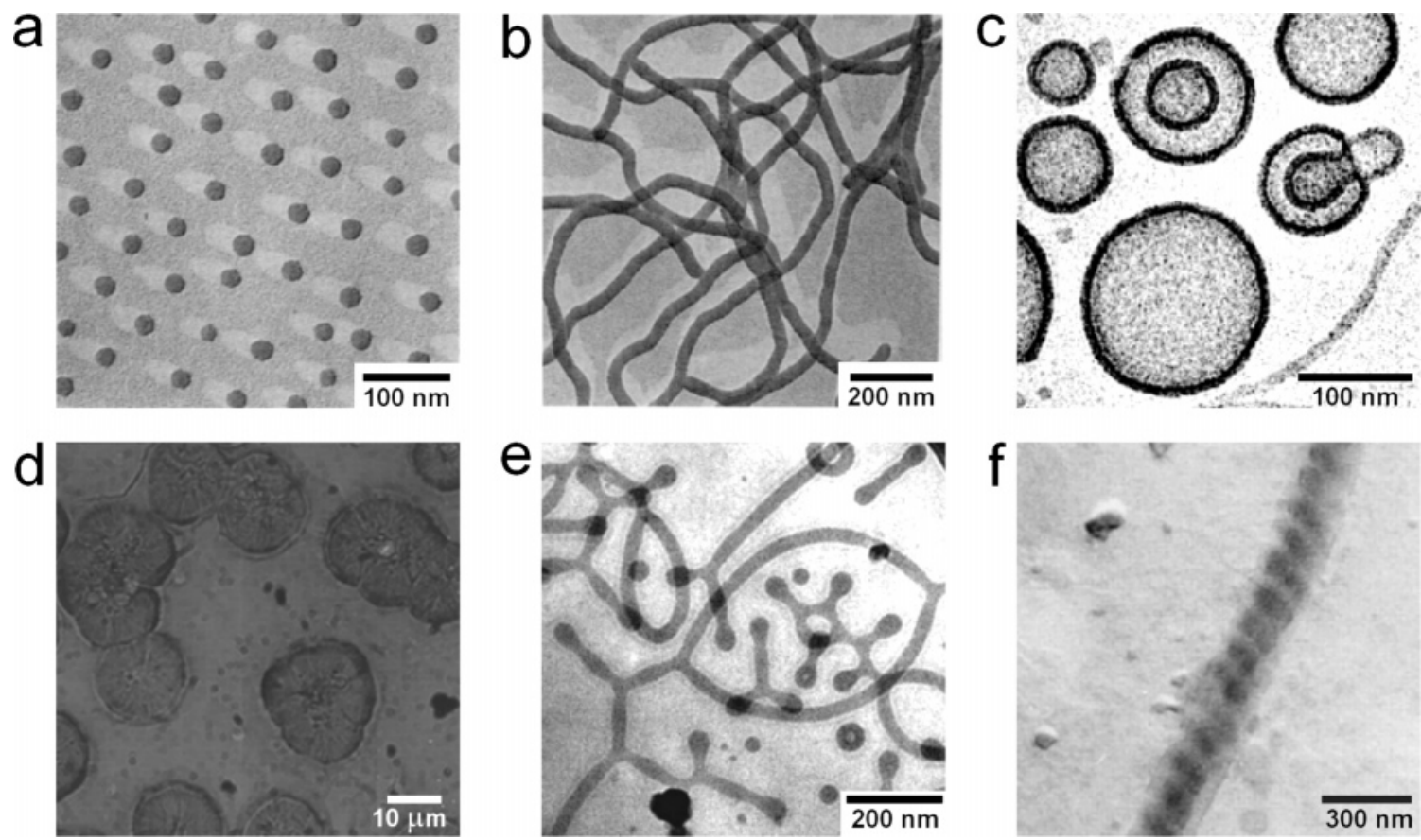

Figure 18. Electron micrographs of various types of morphologies formed by aggregated block copolymers. (a) Micelles and (b) micellar rods from polystyrene- $b$-poly(acrylic acid) (Reproduced with permission from ref 203. Copyright 1999 National Research Council of Canada), (c) vesicles from poly(ethylene oxide)-b-polyethylethylene (Reproduced with permission from ref 204. Copyright 1999 American Association for the Advancement of Science), (d) lamellae from polystyrene- $b$-poly(phenylquinoline) (Reproduced with permission from ref 207. Copyright 1998 American Association for the Advancement of Science), (e) branched wormlike micelles from poly(ethylene oxide)- $b$-polybutadiene (Reproduced with permission from ref 206. Copyright 2003 American Association for the Advancement of Science), and (f) left-hand helices from polystyrene- $b$-poly(L-isocyanoalanyl-L-alanine). (Reproduced with permission from ref 205 . Copyright 1998 American Association for the Advancement of Science.)

referred to as polymersomes. Since the first reports, ${ }^{208,209}$ a large number of examples describing the formation of polymersomes have been published..$^{210-213}$ The structure of vesicle-forming block copolymers can vary from simple coil-coil diblock copolymers and further to rod-coil diblock copolymers to coil-coil and rod-coil multiblock copolymers with and without additional cross-linkable groups (Figure 19).

The advantage of polymersomes over liposomes is their increased stability and the rigidity of their membrane system, which contribute to their increased lifetime. The vast amount of available monomers and the ability to vary the ratio of the two blocks make it possible to tune the properties of the resulting vesicles, for example, vesicle size, polarity, stability, toxicity, etc. In general, however, the permeability of the membranes of block copolymer vesicles is reduced because their thicknesses are higher and their membranes have less fluidic character as compared to liposomes. ${ }^{211}$

In the group of Discher, much effort has been put into establishing the physicochemical properties of polymersomes based on poly(ethylene oxide)- $b$-polybutadiene (PEO-PBD) and poly(ethylene oxide)- $b$ polyethylethylene (PEO-PEE). ${ }^{204,214-216}$ As a first step toward in vivo applications, encapsulation experiments were performed with the proteins myoglobin, hemoglobin, and albumin (Figure 20).

The encapsulation of these proteins was rather straightforward, viz., adding the solid block copolymer to an aqueous solution of the desired solute and waiting for $24 \mathrm{~h}$. The encapsulation efficiencies for the different proteins, however, varied. ${ }^{217}$ The polymersomes appeared to be stable in blood plasma and were observed to be inert to white blood cells and cultured cells. In vivo studies performed with these polymersomes in rats showed that their in vivo circulation times were about 2 times longer than "PEGylated" stealth liposomes $\left(20-30 \mathrm{~h}\right.$ in rats). ${ }^{218}$ These circulation times turned out to be primarily dependent on the PEO block length, rather than on the hydrophobic core-forming block. These experiments clearly show that these PEO-based diblock copolymers have great potential as drug delivery vehicles.

For the controlled release of encapsulated solutes, much effort is being focused on the development of stimuli-responsive vesicles, that is, capsules that are disrupted by changes in their environment. Such a system based on polymersomes was prepared by encapsulating the enzyme glucose oxidase (GOx) within polymersomes of poly(ethylene glycol)- $b$-poly(propylene sulfide)- $b$-poly(ethylene glycol) (PEG-PPSPEG) (Figure 21). ${ }^{219}$

The thioethers in the hydrophobic middle block are converted to more hydrophilic sulfoxides and sulfones upon exposure to an oxidative environment, in that way increasing the hydrophilicity and solubility of the macromolecular amphiphile. GOx converts glucose to gluconolactone, and, in the presence of oxygen, hydrogen peroxide is formed. It was shown that destabilization of the GOx-encapsulating polymersomes occurred upon the oxidative action of the formed hydrogen peroxide when glucose was extra- 

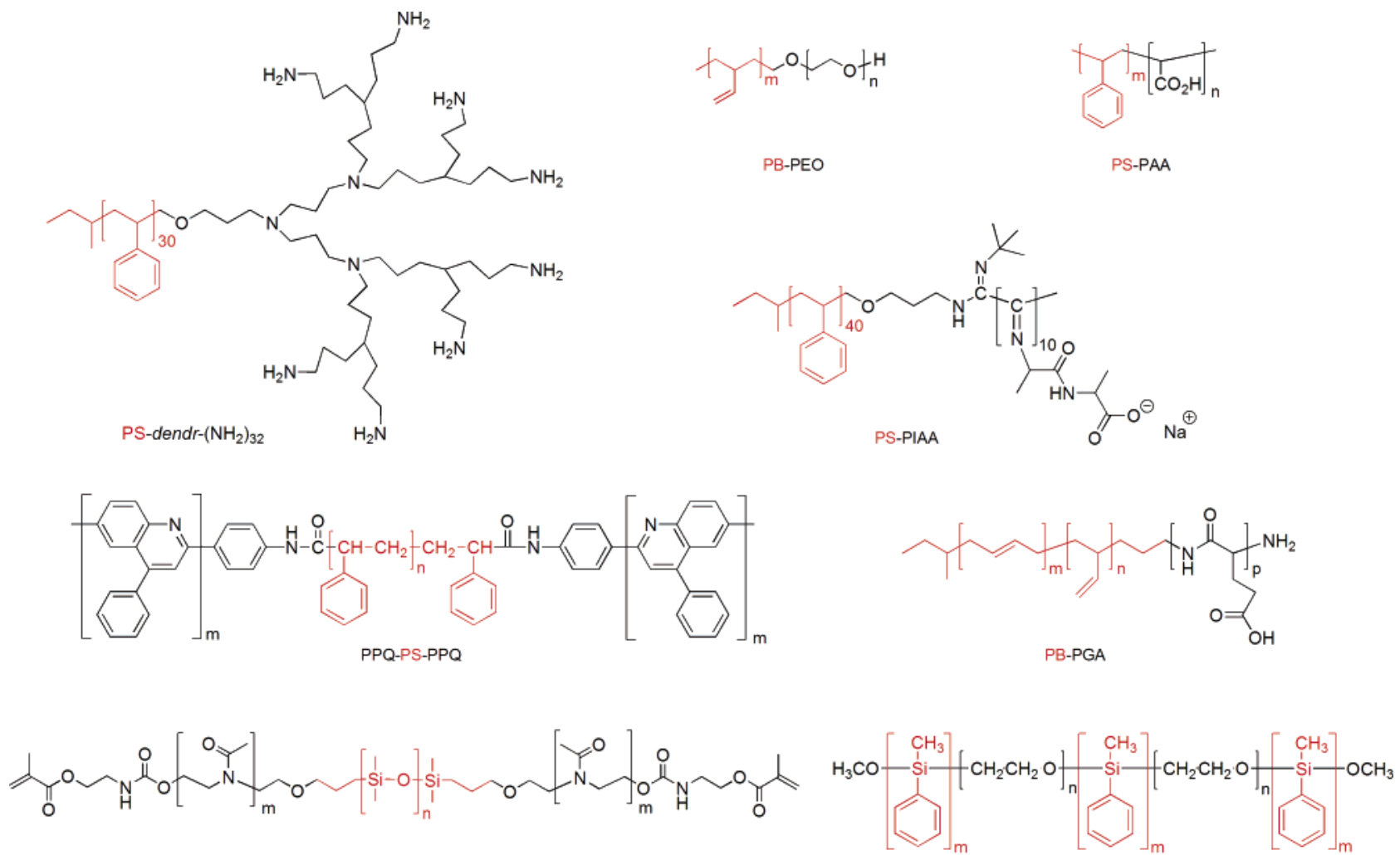

PMOXA-PDMS-PMOXA

PMPS-PEO-PMPS-PEO-PMPS

Figure 19. Structure formulas of various polymersome-forming block copolymers. The hydrophobic blocks are depicted in red.
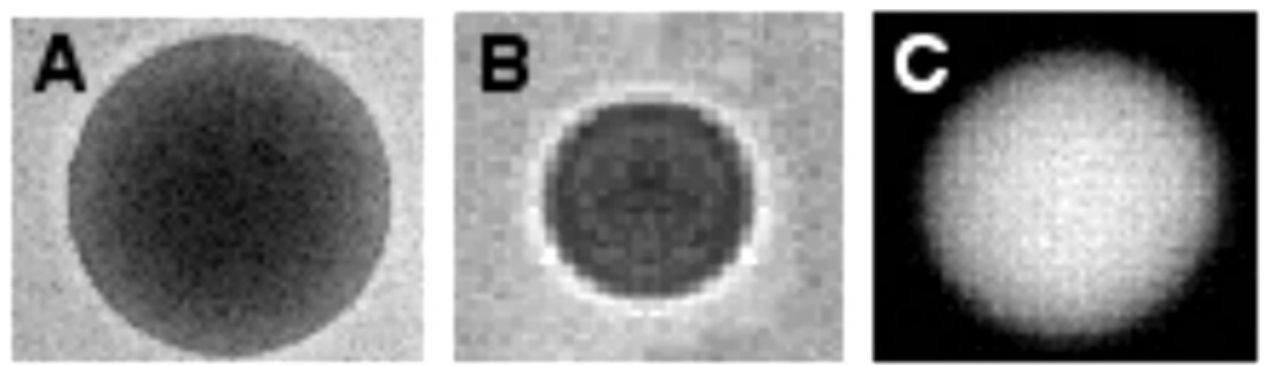

Figure 20. Encapsulation of proteins using block copolymers made of PEO-PEE. (a) A polymersome containing myoglobin (diameter is $15 \mu \mathrm{m}$ ), (b) a polymersome containing hemoglobin (diameter is $5 \mu \mathrm{m}$ ), (c) a polymersome containing fluoresceinlabeled BSA (diameter is $15 \mu \mathrm{m}$ ). (Reproduced with permission from ref 217. Copyright 2001 Wiley.)

vesicularly added. This approach to develop stimulusresponsive vehicles for drug delivery seems feasible, but also appears to be rather limited and complex, because the presence of GOx and glucose is required for every application. Another stimulus-responsive polymersome was developed on the basis of polybutadiene- $b$-poly $\left(\gamma\right.$-L-glutamic acid). ${ }^{220}$ The size of the polymersome molecules could be reversibly altered by changing both the $\mathrm{pH}$ and the ion strength. Bellomo et al. also recently published on $\mathrm{pH}$-sensitive polymersomes, which are based on diblock copolypeptide. ${ }^{221}$ Najafi et al. have prepared di- and triblock copolymers containing anhydride groups from fumaric/sebacic acid and PEG. ${ }^{222}$ The diblock copolymers formed micelles, while the triblock copolymers yielded polymersomes. It was observed that the hydrolytic degradation of the triblock copolymers was slower than that of the diblock copolymers.

The group of Meier has developed a nanoreactor by incorporating the $\mathrm{OmpF}$ channel protein in the membrane and $\beta$-lactamase enzymes inside the water pool of polymersomes from the amphiphilic ABA triblock copolymer poly(2-methyloxazoline)- $b$-poly(dimethylsiloxane)-b-poly(2-methyloxazoline) (PMOXAPDMS-PMOXA) (Figure 22). ${ }^{223}$

The triblock copolymer carried methacrylate groups at both ends, thus allowing cross-linking of the block copolymers inside the membrane upon irradiation of the polymersomes with UV light, resulting in very stable aggregates. Transport of reagents through the membrane was achieved by the nonspecific OmpF channel protein encapsulated in the membrane, which allows passive diffusion of small solutes up to a molecular weight of $400 \mathrm{~g} \mathrm{~mol}^{-1}$. The activity of the encapsulated enzyme $\beta$-lactamase was determined by external addition of the substrate ampicillin to a nanoreactor dispersion in the presence of a starchiodine solution. The ampicillin is hydrolyzed by $\beta$-lactamase to ampicillinoic acid, and only the latter is able to reduce iodine to iodide, resulting in decol- 

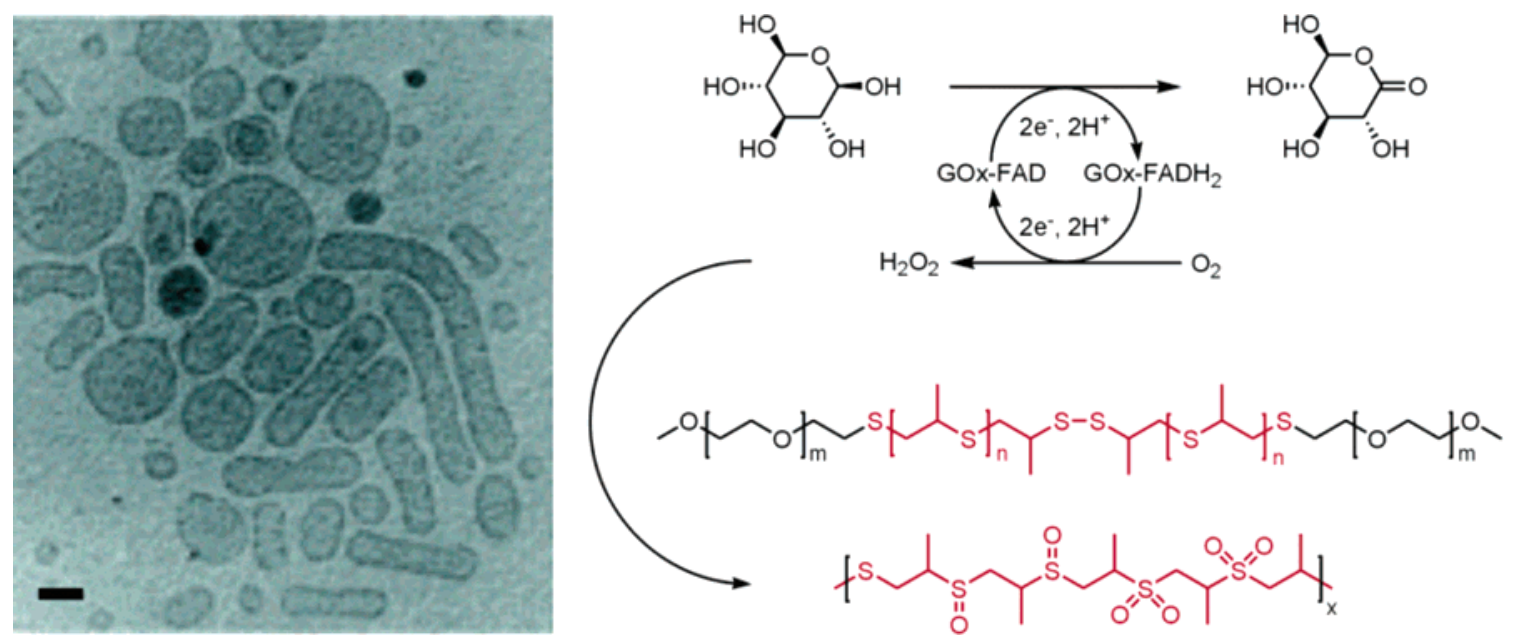

Figure 21. Glucose-responsive capsules based on oxidation-sensitive PEG-PPS-PEG polymersomes encapsulating GOx. Left: Cryo-TEM micrograph of the capsules. Right: Reaction scheme showing the oxidation of glucose which leads to the formation of $\mathrm{H}_{2} \mathrm{O}_{2}$ and consequently the oxidation of the thioethers. A possible repeating unit of PPS after oxidation by $\mathrm{H}_{2} \mathrm{O}_{2}$ is shown. (Reproduced with permission from ref 219. Copyright 2004 American Chemical Society.)
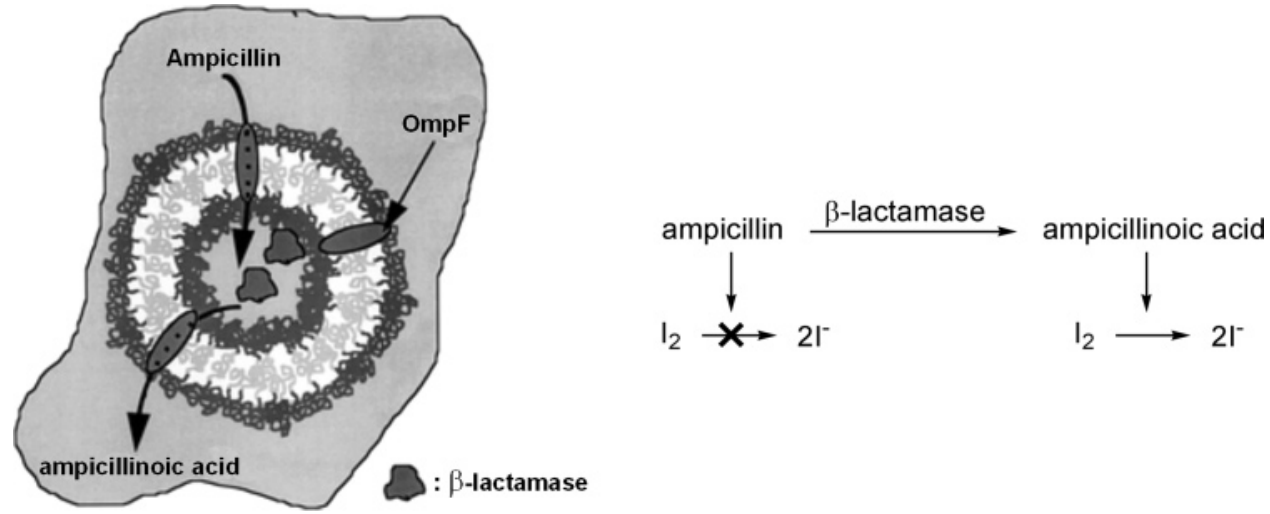

Figure 22. Left: Polymersome nanoreactor developed by the group of Meier. It is composed of a PMOXA-PDMS-PMOXA vesicle encapsulating $\beta$-lactamase enzymes in its inner aqueous compartment and incorporating OmpF channel proteins in its membrane. Right: Oxidation of ampicillin to ampicillinoic acid carried out in the nanoreactor. (Adapted with permission from Figure 2 in ref 223. Copyright 2000 Royal Society of Chemistry.)

orization of the starch-iodine solution, hence allowing the enzyme activity to be monitored spectrometrically (Figure 22).

The same experiment carried out with polymersomes having no membrane channels did not show any enzyme activity during the course of the experiment, indicating that no substrate could diffuse through the membrane. Interestingly, the channel protein showed no decrease in permeability after cross-linking of the membrane.

The OmpF channel protein closes upon increasing the transmembrane potential above a critical value of $100 \mathrm{mV}$. Because molecules above $400 \mathrm{~g} \mathrm{~mol}^{-1}$ are unable to pass the membrane, poly(styrene sulfonate) having sodium counterions was used to increase the potential. The sodium ions will equally distribute between the in- and outside of the polymersome, while the poly(styrene sulfonate) remains outside, in this way establishing a potential, known as a Donnan potential. By this approach, the conversion of ampicillin could be stopped, while dilution of the dispersion or addition of $\mathrm{NaCl}$ opened the channels again, resulting in complete regeneration of the nanoreactor activity. ${ }^{224}$

The growth of calcium phosphate crystals within polymersomes of the same PMOXA-PDMS-PMOXA triblock copolymer has also been studied. ${ }^{225}$ For these experiments, ion-carrying ionophores instead of the OmpF channel protein described in the above studies were incorporated in the polymersome membranes to facilitate the transport of calcium ions. The polymersomes were prepared in the presence of phosphate buffer, and the free phosphate ions were removed by dialysis. This was followed by the addition of a $\mathrm{CaCl}_{2}$ solution. Fractions were taken from the sample, and they were mixed with three different ionophores. The polymersomes were studied by electron microscopy, and after $1 \mathrm{~h}$ the growth of calcium phosphate crystals on the inner membrane could be observed. After $24 \mathrm{~h}$, a considerable amount of space inside the polymersomes was filled with calcium phosphate crystals. In contrast, polymersomes that were not mixed with ionophores did not show crystal formation.

The versatility of PMOXA-PDMS-PMOXA polymersomes was further demonstrated by the injection of DNA by a $\lambda$ phage via a bacterial channel-forming protein incorporated in the polymersome membrane. ${ }^{226}$ The bacterial channel-forming protein served as a receptor for the $\lambda$ phage and triggered the ejection of the phage DNA. It is noteworthy that the bacterial channel-forming protein and $\lambda$ phage func- 


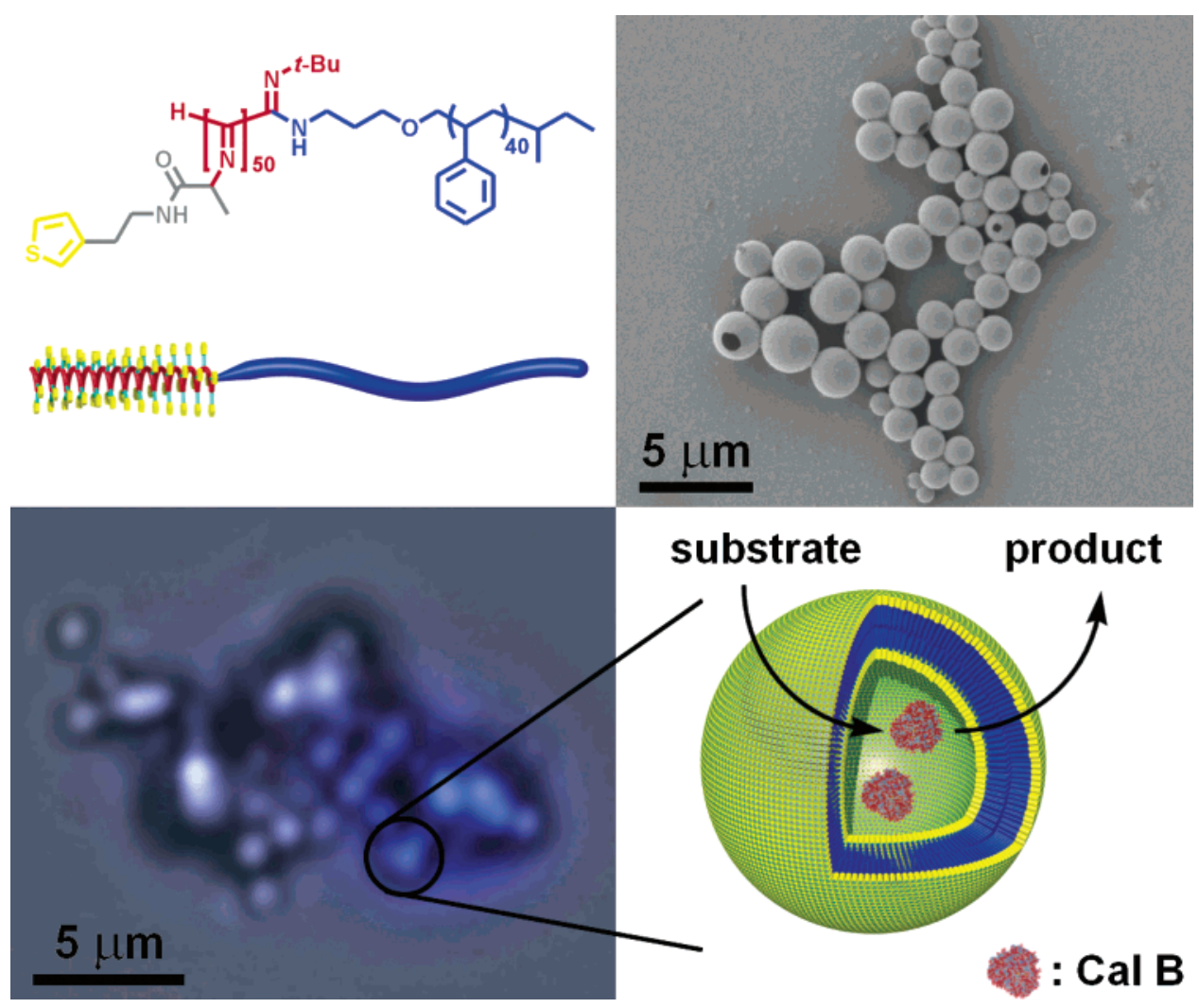

Figure 23. Nanoreactor based on polymersomes of PS-PIAT encapsulating lipase enzymes. Top left: Structure formula and schematic representation of PS-PIAT. Top right: Scanning electron micrograph of PS-PIAT polymersomes. Bottom left: Fluorescence micrograph of the polymersomes containing CAL B enzymes showing fluorescence from the hydrolyzed DiFMU octanoate (see text for details). Bottom right: Schematic representation of the nanoreactor. (Adapted with permission from Figures 1 and 5 in ref 227. Copyright 2003 Wiley.)

tioned well within the unnatural environment of the polymersomes. This showed that complex biological processes can be simulated by using polymeric materials, opening the way toward hybrid cells.

Another type of block copolymer-based nanoreactor was developed by encapsulating Candida antarctica lipase B (CAL B) enzymes inside polymersomes of polystyrene ${ }_{40}-b$-poly(isocyano-L-alanine(2-thiophen-3yl-ethyl)amide) (PS-PIAT) (Figure 23). ${ }^{227}$

This rod-coil diblock copolymer has a rigid helical polyisocyanide block with thiophene side groups which can be cross-linked after vesicle formation, resulting in an electron conductive vesicle membrane. ${ }^{228}$ It was demonstrated that the enclosed CAL B enzymes were still active and that the polymersome membrane was permeable to low molecular weight substrates, for example, 6,8-difluoro-4-methylumbelliferyl octanoate (DiFMU octanoate). Upon hydrolysis of the ester bond of this substrate, a fluorescent coumarin-type of product is formed. Therefore, the enzyme activity can conveniently be monitored quantitatively by fluorescence spectroscopy and qualitatively by a confocal fluorescence microscope. It is noteworthy that good permeability was obtained without the presence of embedded channel proteins or by frustrating the vesicle membrane by the incorporation of compounds. In addition, the membrane of the PS-PIAT vesicles is chiral and therefore potentially selective toward chiral substrates or chiral products.

An interesting example of the application of capsules filled with reactive compounds was presented by White et al. ${ }^{229}$ They developed a polymer with selfhealing capacity by incorporating microcapsules containing dicyclopentadiene monomers and a polymerization catalyst within an epoxy matrix. When the matrix was placed under stress, cracks were formed, which ruptured the embedded microcapsules, thereby releasing the monomers into the crack through capillary forces. Polymerization was initiated by contact with the catalyst, resulting in bonding of the crack faces. Fracture experiments yielded $75 \%$ recovery in toughness as compared to the virgin material.

A different approach toward the preparation of catalytically active polymersomes is the use of "giant" amphiphiles, in which proteins or enzymes act as the polar headgroup of the amphiphile and a synthetic polymer as the apolar tail. Boerakker et al. have developed such a biohybrid amphiphile by reconstituting apo-horseradish peroxidase (apo-HRP) with its cofactor, ferriprotoporphyrin IX, carrying a polystyrene tail (Figure 24a and b). ${ }^{230}$ In water, the enzymepolymer hybrid formed vesicular aggregates with diameters of 80-400 nm (Figure 24c).

Catalytic activity measurements showed that the HRP-polystyrene aggregates were still active; the residual activity, however, was much lower than that of the native HRP. This approach is promising, because other ferriprotoporphyrin IX containing enzymes could also be used to construct giant amphiphiles. The resulting different hybrids can be combined within one aggregate to give a catalytic 


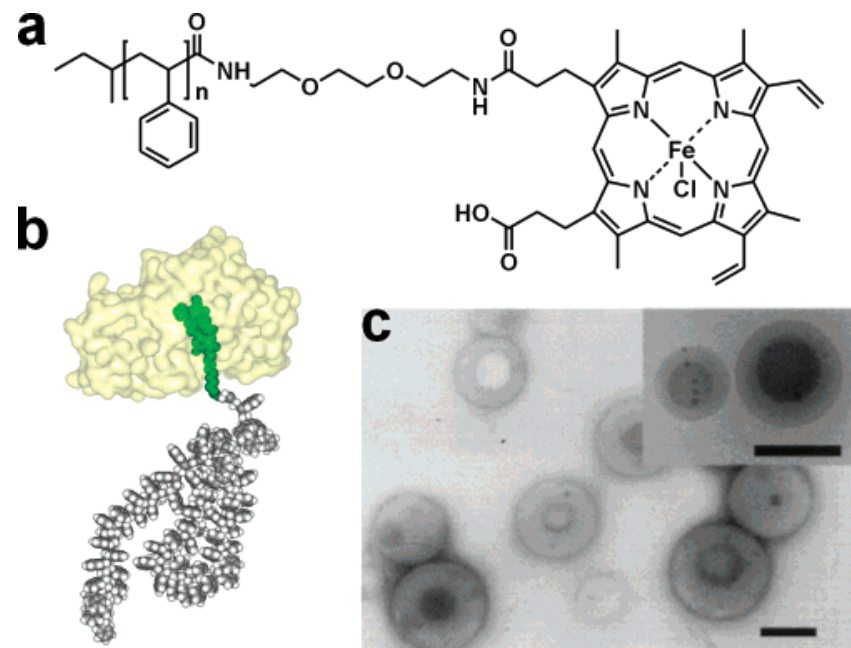

Figure 24. Vesicles formed in water by a biohybrid amphiphile of HRP-polystyrene. (a) Structure formula of the modified cofactor, (b) computer-generated model of the biohybrid, and (c) transmission electron micrograph of the formed vesicles. Scale bars represent $200 \mathrm{~nm}$. (Adapted with permission from Figure 2 in ref 230. Copyright 2002 Wiley.)

system that is capable of performing cascade reactions.

\subsection{Polymer Micelles as Nanoreactors}

A regularly seen morphology for block copolymers is that of a micelle, in which the polar block is on the outside and the apolar block on the inside or vice versa, depending on whether the solvent is polar or apolar. Micelles, which can be spherical, rodlike, or display a hexagonal phase, have a compartment that is capable of accommodating solutes.

The use of micelle-forming amphiphilic block copolymers in the stabilization of metal nanoparticles has been intensively studied, especially by the groups of Antonietti, Möller, and Cohen. ${ }^{200,231-233}$ Not only does the presence of the polymeric shell around the metal particles aid in the prevention of agglomeration and precipitation, it also improves their processability. The block copolymer micelles can indeed be regarded as nanoreactors, because metal nanoparticles are synthesized inside their interior. Homopolymers have also been frequently employed for the stabilization of metal colloids, mainly poly( $N$-vinyl2-pyrrolidone) (PVP). Although the resulting homopolymer-metal particle hybrids are not micelles, they are discussed in this section, because their dimensions and behavior are comparable to the micellar systems presented here. The nanosize dimensions of the metal particles give rise to a number of intriguing electronic, magnetic, optical, and catalytic properties, which is the result of size quantization effects and the high number of surface atoms as compared to the number of atoms in the bulk of the particle. ${ }^{202,231}$ The steps involved in the formation of such metal particles are depicted in Scheme 9.

In Scheme 9, only one approach using metal salts for the formation of polymer-metal hybrids is given, but other approaches have been developed as well. It is also possible to first complex the metal ions to the monomers, then polymerize the monomers, and, finally, induce aggregation of the resulting metalpolymer hybrids. To obtain stable hybrid materials of polymers and inorganic material, there has to be sufficient adhesion between the polymer chains and the metal particles. For that purpose, homopolymers and block copolymers have been synthesized with functional blocks, that is, acidic, basic, or neutral coordinating blocks. The formation of the metal colloids inside the loaded micelles occurs by performing a chemical reaction, typically a reduction. For this purpose, $\mathrm{H}_{2}, \mathrm{NaBH}_{4}, \mathrm{LiAlH}_{4}, \mathrm{LiBEt}_{3} \mathrm{H}$, and hydrazine are commonly applied to prepare nanoparticles of $\mathrm{Ag}$, $\mathrm{Au}, \mathrm{Co}, \mathrm{Cu}, \mathrm{Ni}, \mathrm{Pb}, \mathrm{Pd}, \mathrm{Pt}, \mathrm{Rh}$, and $\mathrm{Zn} .{ }^{200,232}$ The formed metal particles subsequently aggregate to yield larger particles by nucleation and growth processes. Depending on the degree of supersaturation with the metal particle-forming salt, the interfacial tension of the block copolymer/metal particle interface, and the diffusivity of the metal ions, one or more metal nanoclusters are formed within a micelle. ${ }^{200}$ In Figure 25, the effect of supersaturation with the salt $\mathrm{Pd}(\mathrm{OAc})_{2}$ inside diblock copolymer micelles of polystyrene- $b$-poly(4-vinylpyridine) (PS$\mathrm{P} 4 \mathrm{VP})$ is shown. ${ }^{234}$ When the solubilized $\mathrm{Pd}(\mathrm{OAc})_{2}$ was rapidly reduced (high supersaturation), numerous small metal particles were formed within the micellar core, also referred to as the "raspberry" morphology. Slow reduction (low supersaturation), however, led to on average one large particle per micelle, which is called the "cherry" morphology. 234

Not only metal colloids, but also metal oxides, ${ }^{202,235}$ for example, $\mathrm{Fe}_{2} \mathrm{O}_{3}, \mathrm{TiO}_{2}$, and $\mathrm{ZnO}$, and metal sulfides, ${ }^{202,236}$ for example, CdS, $\mathrm{CoS}, \mathrm{CuS}, \mathrm{FeS}, \mathrm{PbS}$, and $\mathrm{ZnS}$, can be formed within micellar nanoreactors. The latter nanoparticles are mostly formed by addition of $\mathrm{H}_{2} \mathrm{~S}$ to the metal precursors inside the nanoreactors, while the former nanoparticles are prepared by oxidation reactions. The optical properties of the metal sulfide semiconducting nanocrystals are very size-dependent, and size control can be obtained by varying the size of the polymer domains. In smaller domains, nanoparticles of lower dimensions are formed, which have absorption edges at smaller wavelengths due to the size quantization effect. Reducing the dimensions of semiconductor nanoparticles, furthermore, results in photogenerated electrons that have higher energies, which can be utilized in photovoltaic devices, and increased optical absorption coefficients. The latter is useful in applications where UV protection is an issue. Micellar fibers of

Scheme 9. Steps Involved in the Preparation of a Metal Nanoparticle Inside a Micellar Nanoreactor
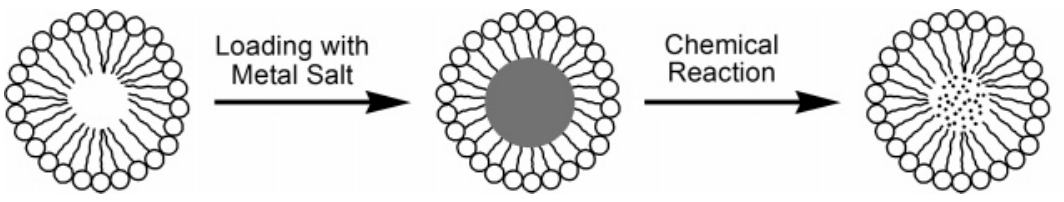

Nucleation and Growth

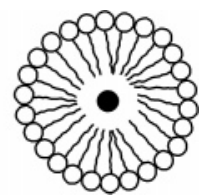



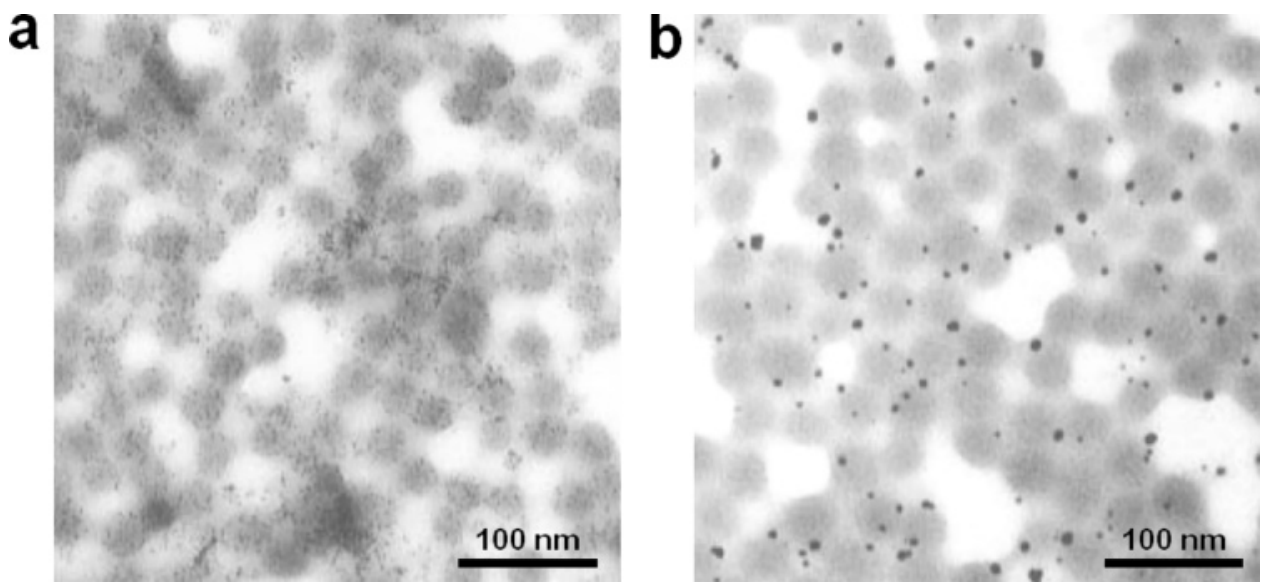

Figure 25. Formation of metal nanoparticles inside block copolymer micelles. (a) Many Pd colloids are formed inside micelles during fast reduction (raspberry morphology). (b) On average, one large Pd colloid per micelle is formed during slow reduction (cherry morphology). (Reproduced with permission from ref 234. Copyright 1997 American Chemical Society.)

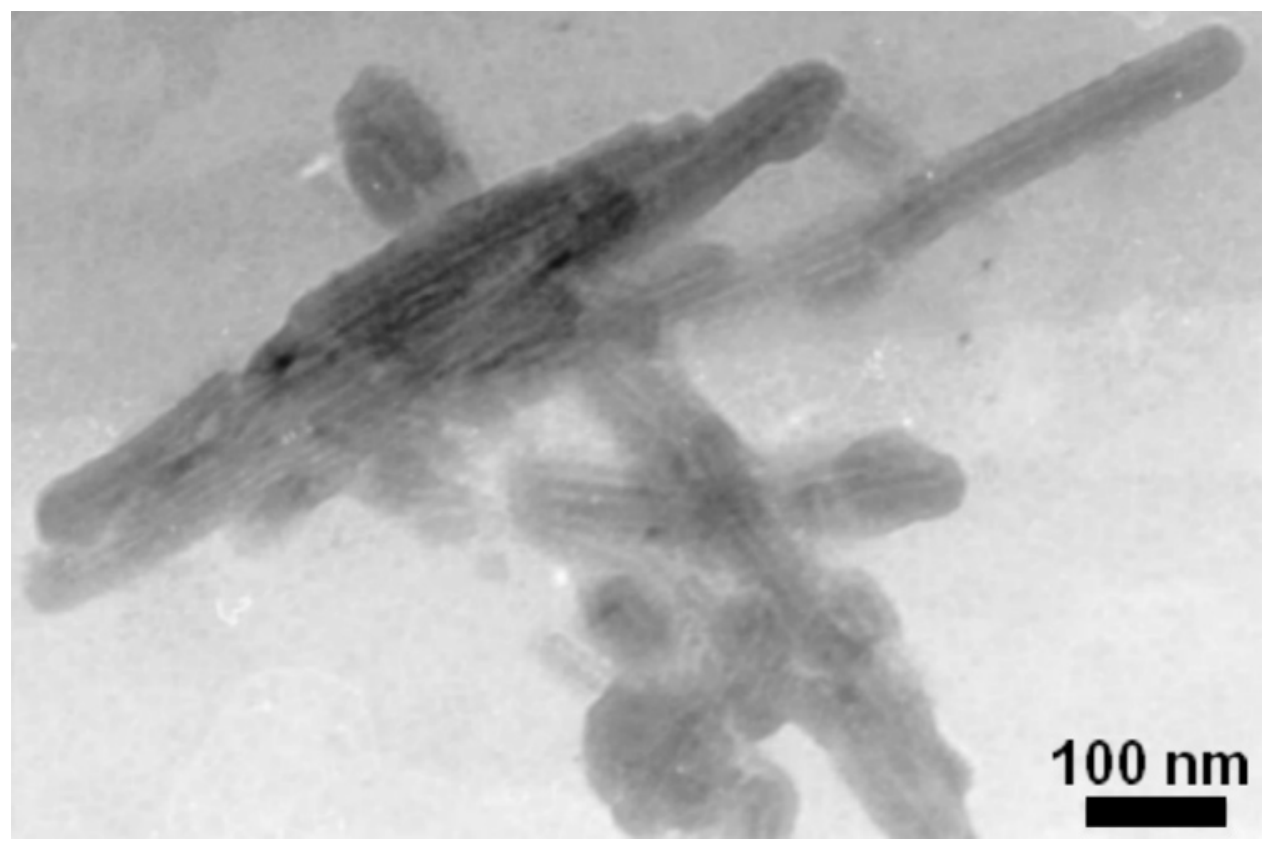

Figure 26. Nanoarrays of Ag within micellar fibers of amphiphilic diblock copolymers of carbosilane dendrimers and polyisocyanopeptides. (Reproduced with permission from ref 237. Copyright 2002 Wiley.)

diblock copolymers of carbosilane dendrimers and polyisocyanopeptides have been applied to prepare silver arrays by clustering $\mathrm{Ag}^{+}$ions inside these fibers (Figure 26). ${ }^{237}$

The polymer micelle-metal hybrid itself can also be considered to be a nanoreactor, because the catalytically active metal colloid is buried inside the micelle core and the reactants have to pass the micellar shell to reach the interior. These hybrid particles combine the advantages of homogeneous and heterogeneous catalysis, because they can be dissolved in organic solvents, due to their polymeric shell, while catalyst recovery is straightforward by performing ultrafiltration or precipitation in poor solvents for the polymer.

So far, polymer micelle-metal hybrids have mainly been applied in the hydrogenation of olefins and acetylenes, where advances have been made in chemo-, stereo-, and regioselective hydrogenations of various substrates. ${ }^{231,238}$ A nice example of chemoselective hydrogenation was presented by $\mathrm{Yu}$ et al. who used Pt nanoparticles stabilized by PVP to reduce the carbonyl group in cinnamaldehyde to cinnamic alcohol, while leaving the double bond intact. ${ }^{239}$ Ethyl pyruvate was reduced enantioselectively to $(R)$-ethyl lactate in $95-98 \%$ enantiomeric excess by Pt colloids stabilized by PVP with cinchonidine as chiral modifier. ${ }^{240}$ Colloidal dispersions of PVP-stabilized $\mathrm{Au} / \mathrm{Pd}$ bimetallic clusters, prepared by successive reduction, were employed by Harada et al. for partial selective reduction of cycloocta-1,3diene to cyclooctene. ${ }^{241}$

Polymer-stabilized metal colloids have also been used in oxidation reactions. The catalytic oxidation of ethylene to ethylene oxide by colloidal Ag catalysts, which were protected by PVP or sodium polyacrylate, was studied by Toshima and co-workers. ${ }^{242,243}$ Both systems had higher catalytic activities than commercial Ag powder, while the sodium polyacrylatestabilized Ag particles gave the best results due to a higher thermal stability. 
Scheme 10. Heck Reaction Catalyzed by Pd-Polymer Hybrids

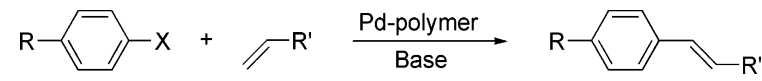

Scheme 11. Suzuki Coupling Reaction Catalyzed by Polymer-Stabilized Pd Nanoreactors

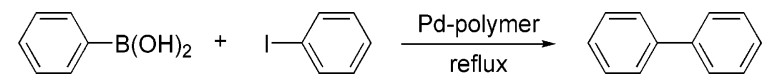

Another field of catalysis in which polymer-metal hybrid nanoreactors have been explored is $\mathrm{C}-\mathrm{C}$ coupling reactions. The first example of methanol carbonylation, which is one of the most important industrial processes, was presented by Wang et al. who applied PVP-stabilized Rh colloids. ${ }^{244}$ The catalysts were used under harsh conditions of $140{ }^{\circ} \mathrm{C}$ and 54 bar, but could still be recycled six times, resulting in an overall turnover number of 19 700/atom Rh. Pd colloids stabilized by either PS-P4VP or PVP have proven to be useful in Heck reactions (Scheme 10). ${ }^{234,245}$

The polymer-stabilized Pd nanoparticles displayed activities comparable to those of low molecular weight $\mathrm{Pd}$ complexes traditionally used in Heck reactions, while having much higher stabilities; turnover numbers (moles of substrate/mole of $\mathrm{Pd}$ ) as high as 100000 have been reported. ${ }^{245}$ The higher stability of the polymer-metal hybrid is also reflected in the high temperature $\left(140{ }^{\circ} \mathrm{C}\right)$ at which these reactions were carried out.

El-Sayed and co-workers have used the Suzuki coupling as a test reaction to investigate the effect of the polymeric stabilizers on both the catalytic activity and the stability of Pd colloids (Scheme 11). ${ }^{246}$

They prepared encapsulated Pd nanoparticles with the help of three different polymers: a PAMAM dendrimer, polystyrene- $b$-poly(sodium acrylate), and PVP. All three nanoreactor systems were efficient catalysts for the Suzuki reaction between aryl boronic acids and aryl halides. It was found that a strong interaction between the metal particle and the polymer resulted in a loss of catalytic activity. Lee et al. investigated the same type of reaction in water using aggregates of rod-coil triblock copolymers as micellar nanoreactors. ${ }^{247}$ At ambient temperatures, the Suzuki cross-coupling reaction of aryl halides and aryl boronic acids was performed in the absence of organic solvents, resulting in a potentially environmentally friendly reaction process.

Micelle-forming polymers have been employed to encapsulate enzymes. Micelle-like aggregates built up from diblock copolymers of ( $N$-acetylimino)ethylene and ( $N$-pentanoylimino)ethylene were capable of encapsulating horseradish peroxidase, lipase $\mathrm{OF}$, and lipase P. Interestingly, the hydrolytic activities of the lipases in aqueous solutions increased by ca. $30 \%$ as compared to the free enzyme. ${ }^{248}$ Even in watersaturated organic solvents, the enzymes showed enhanced activities. Harada and Kataoka have published several papers on supramolecular assemblies of micelle-forming poly(ethylene glycol)-poly(aspartic acid) block copolymers and chicken egg white lysozyme. ${ }^{249,250}$ The polyion complex (PIC) micelles could be reversibly formed and dissociated by changes in the ionic strength, simply by varying the $\mathrm{NaCl}$ concentration (Figure 27). ${ }^{251}$

Lysozyme entrapped within the core of the micelles showed no enzymatic activity, but upon an increase in the ionic strength the micelles dissociated, thus releasing the enzymes, and enzymatic activity was detected. A reduction of the ionic strength resulted in complete inhibition of the enzymatic activity. The dissociation of the PIC micelles to obtain enzyme activity is not a nanoreactor behavior, but Harada and Kataoka also performed experiments using the substrate $p$-nitrophenyl-penta- $N$-acetyl- $\beta$-chitopentaoside, and they observed conversion within the core of the micelles. ${ }^{252}$ The apparent enzymatic activity of the entrapped enzymes was higher than that of the free enzymes, which was attributed to accumulation of substrate in the corona of the micelles. Applying a pulsed electric field to the polymerenzyme hybrids above a critical potential reduced the enzymatic activity to that of the free enzyme, presumably due to a minute change in the local microenvironment in the core of the micelles. ${ }^{253}$ The enzymatic activity was fully restored when the electric field was shut-off.

As described above, giant amphiphiles of polymers and enzymes can be prepared by reconstituting apoenzymes with its cofactor carrying a polymer tail. ${ }^{230}$ Velonia et al. have constructed another type of biohybrid amphiphile by specific attachment of a polystyrene block to a reduced disulfide bridge of CAL $B$, exposed on the outer surface of the enzyme. ${ }^{254}$
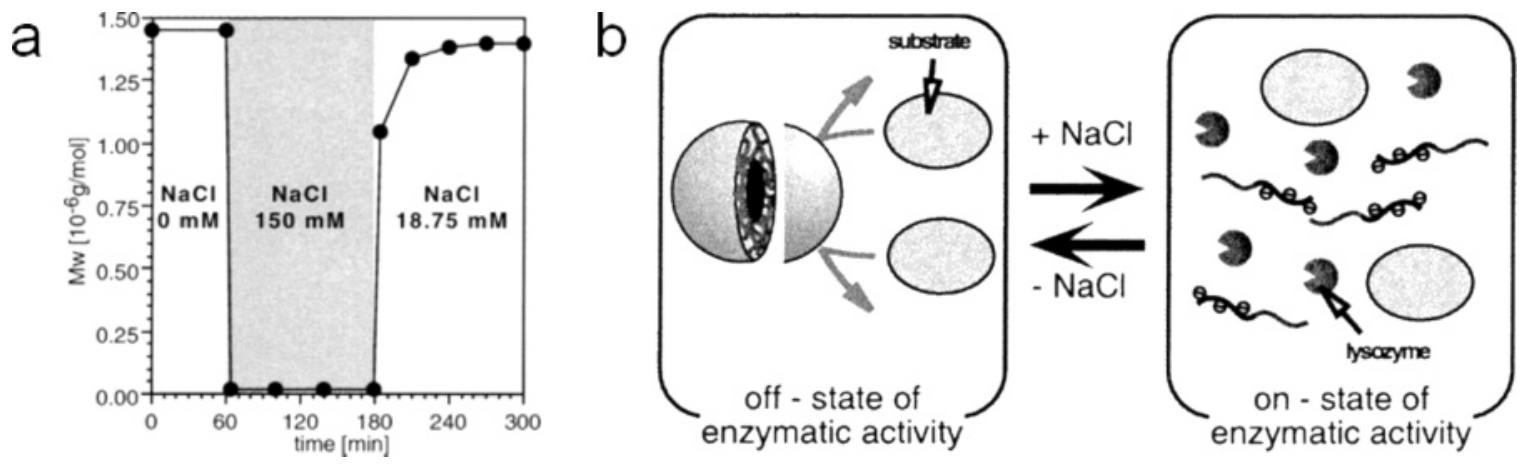

Figure 27. On-off control of enzymatic activity of PIC micelles with entrapped lysozyme enzymes. (a) Reversible formation and dissociation of PIC micelles through a change in NaCl concentration. (b) Schematic model of on-off control of enzymatic activity through reversible formation of PIC micelles. (Reproduced with permission from ref 251. Copyright 1999 American Chemical Society.) 
TEM studies of aqueous dispersions of PS-CAL B giant amphiphiles revealed the presence of micrometer long fibers that were built up from bundles of micellar rods. Catalysis experiments on these enzyme-polymer hybrids revealed that the enzymes retained activity, but it was notably reduced when compared to the native enzyme. Although this is not a nanoreactor, this approach of making catalytically active giant amphiphiles is a promising strategy in the preparation of ensembles of different enzymes and has potential for the construction of multicomponent nanoreactors in which substrates are converted in several cascade-like steps into the desired product(s).

Micelles in which emulsion polymerizations are carried out can also be considered as nanoreactors. Apart from low molecular weight surfactants, ${ }^{127}$ micelle-forming block copolymers have been applied for this purpose. Jang and Ha have used poly(oxyethylene)- $b$-poly(oxypropylene)- $b$-poly(oxyethylene) to make hollow polystyrene nanospheres. ${ }^{255}$ Mini-emulsion polymerization has also proven to be a valuable tool for the fabrication of polymeric capsules. ${ }^{256}$ Mini-emulsions are stable emulsions consisting of droplets of $50-500 \mathrm{~nm}$ in diameter created by shearing a mixture of oil, water, surfactant, and a highly hydrophobic compound. The hydrophobic compound prevents Ostwald ripening, while the surfactant stabilizes the droplets against collisions. ${ }^{257}$ This process allows the preparation of latex particles having cavities with control of the particle size, cavity volume fraction, and structure..$^{258}$ The differences in the hydrophilicity of the oil and the polymer proved to be the driving force for capsule formation. Although mini-emulsion polymerizations do not involve the formation of micelles, it is an interesting technique to prepare hollow polymeric capsules with broad applicability.

\subsection{Unimolecular Nanoreactors}

The dynamic nature of polymeric micelles makes them sensitive to environmental conditions. To overcome this, covalent systems such as dendrimers, hyperbranched polymers, and star polymers have recently been employed, and they have found application as stabilizers in the formation of nanoparticles, recoverable metal catalysts and nanoreactors. These unimolecular compounds are, to some extent, comparable to micelles because they possess an inner compartment capable of accommodating guest molecules, the main difference being that they are not dynamic assemblies. Although the above-mentioned types of polymers are not self-assembled structures, we will discuss them briefly in this review, because of their similarity to polymer micelles.

A dendrimer is a single molecule with a central core from which in a regular fashion branches emerge radially. The overall shape of dendrimers and the existence of cavities inside these compounds are topics of controversy. Most dendrimers are flexible structures, but they adopt a globular shape at a certain generation, which results in an increase in their rigidity. ${ }^{259}$ The use of dendrimers in catalysis is well documented in the literature and is described
Scheme 12. Three Different Types of Catalytically Active Dendrimers: (a) Dendrimer with a Catalytically Active Core, (b) Free-Energy Driven Dendrimer Nanoreactor, and (c) Catalytically Active Metal Nanoparticle Stabilized Inside a Dendrimer

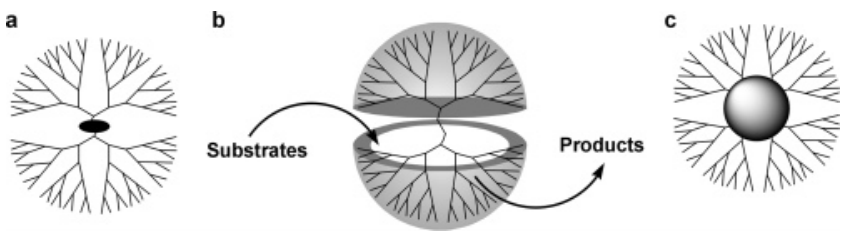

in a number of reviews. ${ }^{259-265}$ Covalently attached catalytic sites in dendrimers can be present at the periphery, at intermediate places, or can constitute the center of the dendrimer. In the last case, the catalytic site is protected by the dendritic branches, resulting in an enzyme mimic. In general, three types of dendrimer nanoreactors can be distinguished: (i) dendrimers with a catalytically active core, ${ }^{261,262}$ (ii) free-energy driven dendrimer nanoreactors, which are not directly involved in the reaction, ${ }^{264}$ and (iii) dendrimers that stabilize a catalytically active metal nanoparticle (Scheme 12). 260

The results obtained with these types of dendrimer nanoreactors in catalysis vary greatly, but in general the dendrimers have an added value, that is, by stabilizing the catalytically active center, by increasing the stereo- and regioselectivity of the reaction, and by allowing easy recovery of the catalyst, for example, by filtration or precipitation. ${ }^{261}$

Hyperbranched and star polymers are cheaper alternatives of dendrimers, because these threedimensional macromolecular structures can be prepared more easily using conventional polymerization methods. ${ }^{266-268}$ In a fashion similar to block copolymer micelles, it has recently been demonstrated by several groups that these hyperbranched and star polymers are capable of encapsulating catalytically active metal complexes and nanoparticles within their cores (Figure 28). ${ }^{268-271}$

These nanoreactors were applied in a wide variety of catalytic processes, for example, the hydrogenation of cyclohexene, ${ }^{269}$ a double Michael addition, ${ }^{270}$ a Heck reaction, ${ }^{268}$ and the oxidation of alcohols to ketones,${ }^{271}$ thus showing that these macromolecular structures are very versatile and can be used in a broad scope of reactions. As in the case of block copolymer micelles, the recovery of polymer-metal hybrids from the reaction mixture is easy using dialysis or precipitation, while they have the advantage of an enhanced stability due to their less dynamic nature.

\subsection{Other Polymeric Systems as Nanoreactors}

Numerous papers have been published dealing with systems that are potential nanoreactors. We will discuss below some systems that have a good chance of being transformed into a working nanoreactor. In this section also polymeric nanoreactors will be discussed that cannot be classified under the subjects of the previous sections.

Chernyshov et al. have used a chelating diblock copolymer, polystyrene- $b$-poly $(m$-vinyl-triphenylphos- 

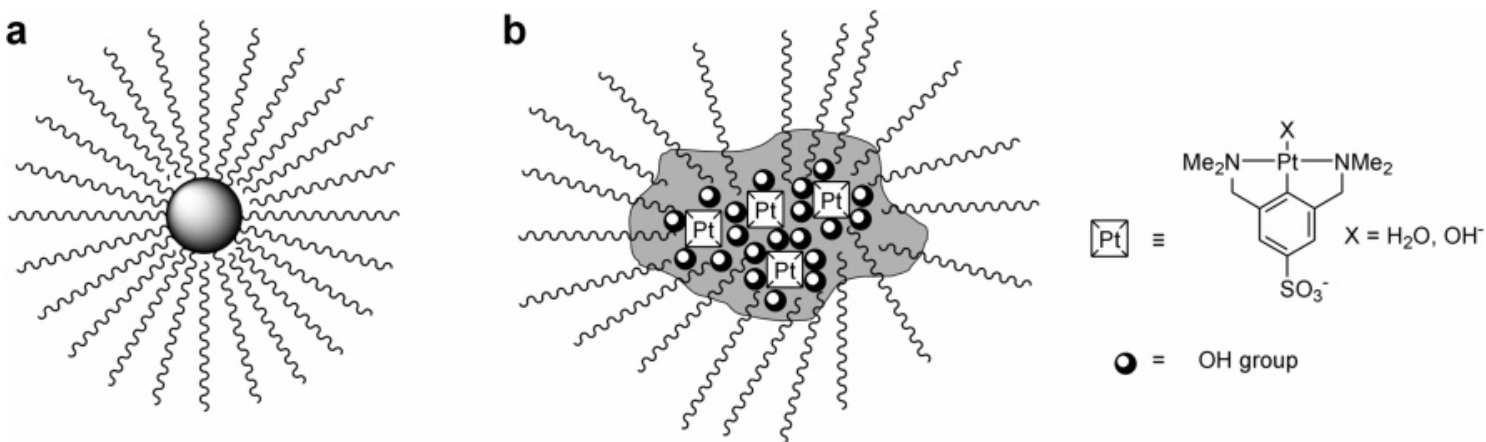

$\mathbf{0}=\mathrm{OH}$ group

Figure 28. Catalysis inside star and hyperbranched polymers: (a) star polymer encapsulating a catalytically active nanoparticle, ${ }^{268}$ and (b) hyperbranched polyglycerol nanocapsule with catalytically active pincer Pt complexes. ${ }^{270}$

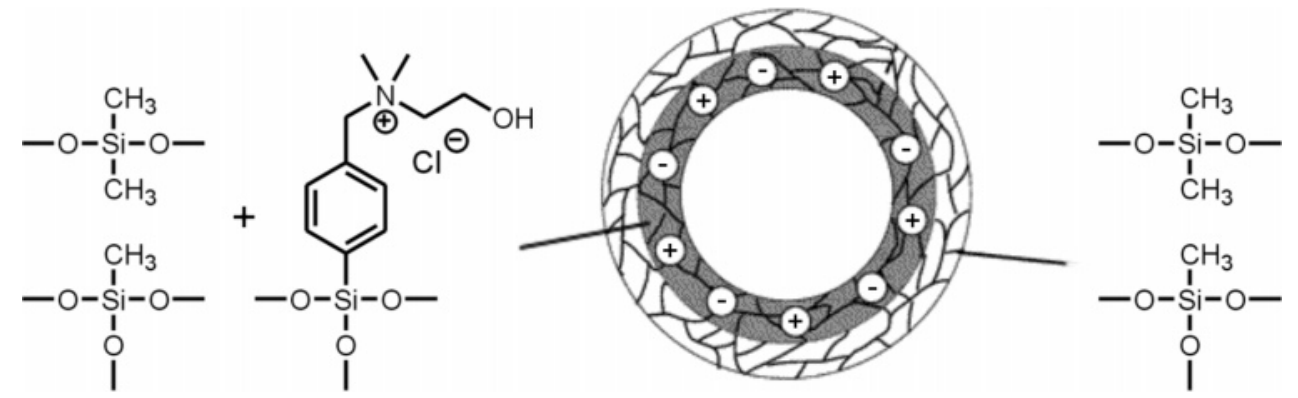

Figure 29. Schematic picture of the hollow amphiphilic nanoreactor that was used by Jungmann et al. for the synthesis of metal colloids. The gray part consists of dimethylsiloxane, methylsiloxane, and a quaternary ammonium salt; the outer shell is built up from dimethylsiloxane and methylsiloxane only. (Adapted with permission from ref 273 . Copyright 2003 American Chemical Society.)

phine) (PS- $b$-PPH), to prepare polymeric $\mathrm{PdCl}_{2}$ complexes. ${ }^{272}$ They found that this hybrid system selfassembled to yield finite structures upon dispersion in THF; no gels were formed. Depending on the ratio phosphine/Pd, different morphologies were obtained, that is, disklike micelles, uni- and multilamellar vesicles, and "perforated hulls". Although not described by the authors, these aggregates might be useful catalysts in Heck reactions or other processes that require $\mathrm{Pd}$, with the advantage that the aggregates can be easily recovered by filtration or precipitation.

A variety of other systems have also been developed for the construction of nanoreactors. Jungmann et al. have prepared hollow amphiphilic poly(organosiloxane) nanospheres by sequential condensation of silanes and have used them as nanoreactors for the synthesis of $\mathrm{Ag}, \mathrm{Au}$, and $\mathrm{Pd}$ nanoparticles (Figure 29). ${ }^{273}$

After the hollow poly(organosiloxane) nanospheres were loaded with salts of the noble metals, reduction was performed with $\mathrm{LiBEt}_{3} \mathrm{H}$, resulting in the formation of $2-5 \mathrm{~nm}$ size metal colloids.

Polymer containing hollow spheres can also be prepared in a self-assembling approach, viz., by assembling polymers around spherical colloids. ${ }^{274}$ This method, referred to as layer-by-layer (LbL) technique, allows the formation of nanocapsules with a well-defined constitution by coating the colloidal templates with alternating layers of polyanions and polycations. ${ }^{275}$ The templates can be removed by changing the $\mathrm{pH}$ or by using solvents selective for the templates. ${ }^{276}$ These hollow particles have recently been applied as nanoreactors for a variety of reactions. ${ }^{277}$ Adopting a ship-in-a-bottle approach, hollow polymeric capsules were loaded with different monomers, for example, styrene sulfonate, and consecutively polymerized. The capsule wall proved permeable for the monomers, whereas the polymers were trapped inside. In this way, the physicochemical properties of the capsule interior could be varied over a broad range (ion strength, $\mathrm{pH}$, viscosity, etc.). In a similar manner, the cationic dye 1,1'-diethyl-2,2'cyanine (DEC) was crystallized inside polymeric capsules that contained poly(styrene sulfonate) (PSS). ${ }^{278}$ The PSS was introduced inside the capsules by the ship-in-a-bottle approach (Figure 30 ).

The formed fluorescent DEC-PSS aggregates were highly photosensitive, and light irradiation resulted in destruction of the aggregates and redistribution of PSS inside the capsules. Addition of tetraphenylborate ions resulted in precipitation of fluorescent

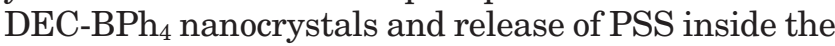
polymer shells.

The versatility of the LbL technique was further demonstrated by coating human erythrocytes with alternating layers of polyanions and polycations. ${ }^{279}$ A protein destruction treatment was carried out to decompose the cytoplasmic proteins of the erythrocytes, which were removed by centrifugation or filtration. The resulting shells were used for the controlled precipitation or crystallization of organic and inorganic materials. In another biomimetic approach, $\mathrm{CaCO}_{3}$ was synthesized exclusively inside micrometer-sized polyelectrolyte capsules. ${ }^{280}$ Urea hydrolysis, catalyzed by urease, led to the fermentative formation of $\mathrm{CO}_{3}{ }^{2-}$ ions and the precipitation of $\mathrm{CaCO}_{3}$, which completely filled the capsule interior. The LbL approach was also adopted by Ghan et al. to polymerize phenols within polyelectrolyte micro- 


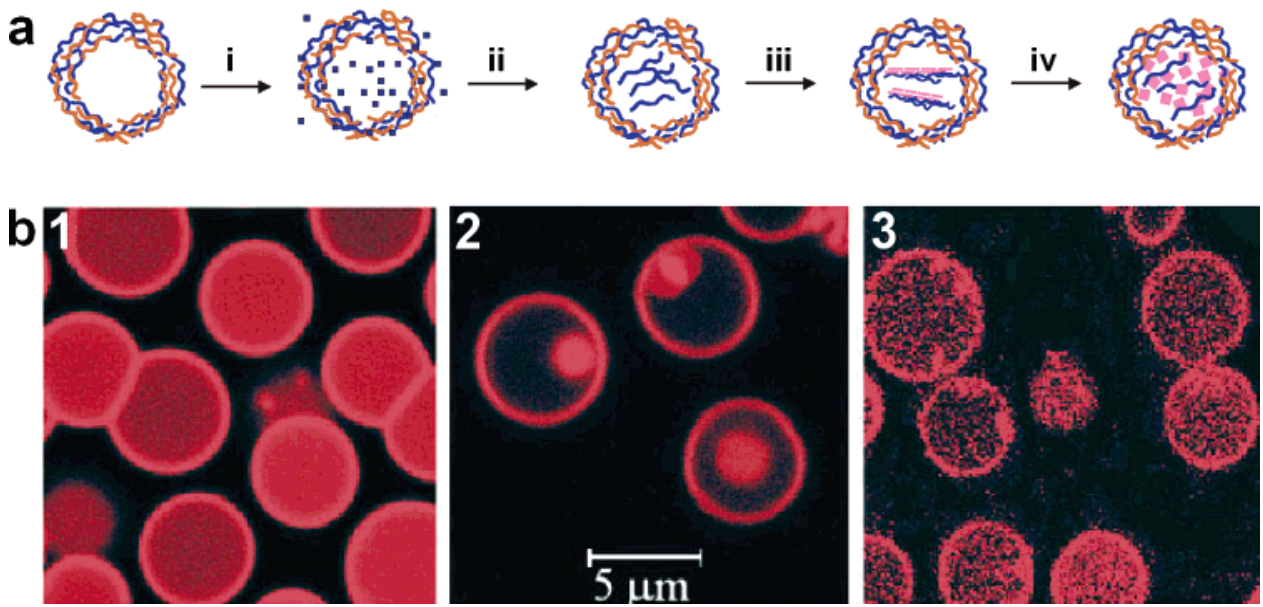

Figure 30. (a) Steps involving the crystallization of DEC inside polymeric capsules: (i) loading of the shell with styrene sulfonate, (ii) polymerization of styrene sulfonate inside the capsule, (iii) polymer-induced formation of DEC-PSS aggregates, (iv) precipitation of DEC crystals and the release of free PSS within the polymer shell. (b) Confocal laser scanning micrographs of (1) capsules containing styrene sulfonate (visualized using rhodamine 6G), (2) fluorescence arising from DEC-PSS aggregates formed inside the capsules, (3) destruction of the DEC-PSS aggregates by laser light. (Adapted with permission from ref 278. Copyright 2003 Wiley.)
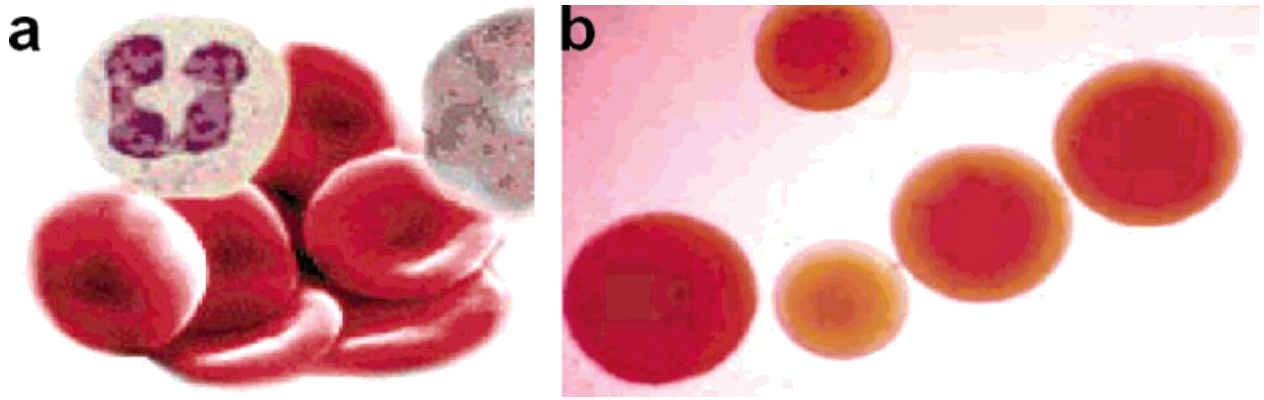

Figure 31. Optical micrographs of (a) red blood cells $(7 \mu \mathrm{m}$ in diameter) and (b) artificial red blood cells containing hemoglobin and enzymes. (Reproduced with permission from ref 282. Copyright 2003 Blackwell Publishing.)

capsules. ${ }^{281}$ After formation, horseradish peroxidase (HRP) could be loaded inside the hollow shells by adjusting the $\mathrm{pH}$ of the solution to 4.0 , resulting in an increase in the shell wall permeability which was large enough to allow HRP to diffuse inside. An increase of $\mathrm{pH}$ to 8.5 closed the capsule walls again, entrapping the HRP enzymes. Polymerization of 4-(2aminoethyl)phenol hydrochloride catalyzed by HRPcontaining capsules in the presence of $\mathrm{H}_{2} \mathrm{O}_{2}$ gave easily detectable fluorescent polymers. The capsule walls turned out to be selective: the monomer and $\mathrm{H}_{2} \mathrm{O}_{2}$ were able to diffuse in and out, whereas the polymer remained inside the capsule. These experiments show that the hollow capsules prepared by the LbL technique create many new possibilities for the synthesis of composite materials under biologically friendly conditions.

For the development of blood substitutes, polymers have been applied to encapsulate hemoglobin, and some of these systems are already in clinical trials (Figure 31). ${ }^{282}$

Although the first attempts of making artificial blood cells date back to the 1950 s, only in the 1990 s was serious attention given to these systems in connection with studies aimed at avoiding the risks of HIV infection. The use of biodegradable polylactide allows one to encapsulate the whole content of red blood cells, and the amount of hemoglobin that can be captured matches that of red blood cells. Ad- ditional enzymes can be encapsulated in these systems, while the products of reactions taking place inside the nanocapsules can diffuse out and, therefore, do not accumulate. Furthermore, reducing agents from the plasma can also diffuse into the nanocapsules where they reduce methemoglobin to oxygencarrying hemoglobin. Animals have been infused with these nanocapsules to the extent of one-third of their total blood volume. Higher circulation lifetimes were obtained with ultrathin membrane nanocapsules containing hemoglobin and enzymes based on poly(ethylene glycol)-polylactide (PEG-PLA). ${ }^{283}$ The PEGPLA nanocapsules were optimized by using polymerized hemoglobin, higher molecular weight PLA, higher concentrations of PEG-PLA, and cross-linking of the PEG-PLA nanocapsules.

Other polymeric systems have also been employed for the fabrication of nanoreactors, but because of their preparation procedure they do not fall within the scope of this review. However, to be complete, they will be briefly discussed. The underlying idea of immobilizing catalysts within polymeric supports is to simplify their separation from the reaction mixture and to allow recycling of the catalyst. Although incorporated within the polymer matrix, the catalyst still has to be mobile to have a rate of reactivity comparable to that of the catalyst in homogeneous solution. Zeolites and sol-gel materials are very interesting supports for the immobilization 
of catalysts. Their precursors can be mixed at the molecular level, thereby allowing the creation of homogeneous solid-state systems.

The sol-gel process is a versatile method for the preparation of heterogeneous catalysts that behave as homogeneous catalysts. The advantages of a homogeneous system, that is, high activities and selectivities, can be combined with the advantages of a heterogeneous system, that is, facile separation and recovery of the catalyst. ${ }^{284}$ To ensure sufficient mobility, flexible spacers are used to link the catalytic centers to the solid support. These materials can be formed under mild conditions, in that way allowing the incorporation of sensitive catalysts. The versatility in the synthesis allows the preparation of solgels with organic acids and bases, or with metal complexes as the reactive centers. Sol-gel catalysts have been successfully employed in a vast series of reactions, generally with modest to excellent results. $^{284}$

An interesting finding is that sol-gel materials can encapsulate biologic components, while keeping them in a fully functional state. In this way, not only proteins, enzymes, and antibodies have been entrapped within ceramic matrixes, but also DNA and RNA, and even living cells. ${ }^{285,286}$ Interestingly, the structural integrity of the cells remained intact, and molecular recognition, catalysis, and reproduction capabilities were preserved as well. Applications of such hybrid systems can be found in the fields of catalysis, sensors, separation technology, diagnostics, and electronics.

Zeolites are microporous crystals that consist of aluminosilicate tetrahedrons, which form a secondary structure of channels, cavities, and pores. In the petrochemical industry, zeolites are applied on a large scale in cracking processes. Inside the cavities of zeolites, catalytically active metal complexes that are larger than the pores can be incorporated. These types of zeolite/guest complexes are called ship-in-abottle complexes. ${ }^{287}$ Such complexes can be prepared in two different ways: (i) assembly of the metal complex within the cavity by externally adding the metal ions and the ligands, and (ii) synthesis of the zeolite around the preformed metal complex, also referred to as the build-bottle-around-ship approach. A drawback of the first method is the presence of free ligands and metal ions, resulting in ill-defined systems. Depending on the type of zeolite, the cavities can be used to host large molecules such as bis(salicylidene)ethylenediamine (salen), ${ }^{288}$ phthalocyanine (Figure 32), ${ }^{287,289}$ and porphyrin. ${ }^{290}$

A range of reactions can be performed using shipin-a-bottle complexes, that is, oxidations, ${ }^{291}$ hydrogenations, ${ }^{292}$ and acid-catalyzed isomerization and disproportionation reactions. ${ }^{293}$

Recent advances in organic crystal engineering, particularly the rational design of complex solid architectures through supramolecular preorganization, have renewed interest in topochemical reactions. ${ }^{294-299}$ Although not many catalysis experiments have been carried out with these organic crystals, it is worth mentioning the possibility in this review. Organic analogues to zeolites and mesoporous

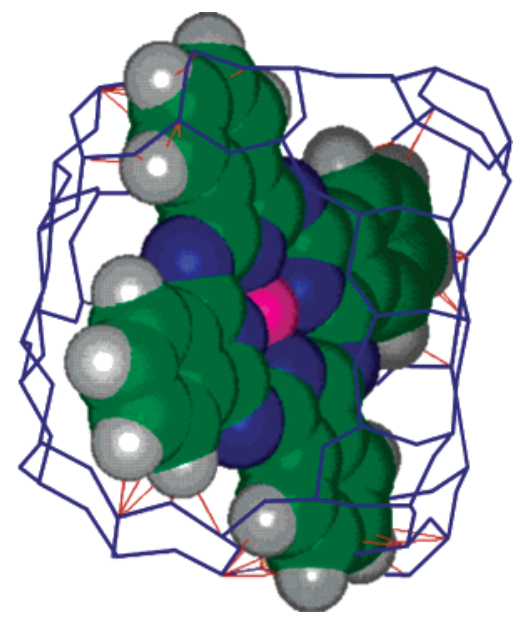

Figure 32. Molecular model showing $\mathrm{Cu}$-phthalocyanine encapsulated inside zeolite Y. (Reproduced with permission from ref 287. Copyright 2004 Wiley.)

sieves were developed by Miller et al. ${ }^{300}$ They made catalytically active, nanoporous organic materials based on cross-linked lyotropic liquid crystals and demonstrated their use as efficient catalysts in Knoevenagel condensations. Dewa et al. prepared hydrogen-bonded solid hosts from 9,10-bis(3,5-dihydroxyphenyl)anthracene and demonstrated that they catalyze the Diels-Alder reaction of acrylaldehyde and 1,3-cyclohexadiene. ${ }^{301}$ Liquid crystals and organic crystals have also been applied as constrained geometries for controlled polymerizations, resulting in interesting polymeric architectures. ${ }^{302}$

\section{Biomacromolecular Nanoreactors}

A new approach to the formation of nanostructures has emerged from studies at the interfaces between biology, chemistry, and materials science. ${ }^{303} \mathrm{~A}$ number of groups are rapidly expanding the repertoire of biological scaffolds for nanochemistry. Reviews such as the ones from Niemeyer, ${ }^{304}$ Young et al., 303 Belcher et al., ${ }^{305}$ and Kirshenbaum et al. ${ }^{306}$ describe thoroughly these new approaches, which use biological molecules and assemblies from them for applications in nanoscience. The current interest in these biosystems stems from the fact that they display a high degree of organization, are often easy to modify, and occur in well-defined self-assembly motifs. ${ }^{307}$

In this part of the review, it is shown that organized biomolecular architectures can serve as nanoreactors or nanotemplates for crystallizations and other reactions. Furthermore, it is shown that protein capsids can be modified with new chemical functionalities and can be used as templates for nanoscale constructions. Finally, it is discussed how self-assembled protein cages can provide spatially welldefined host systems.

\subsection{Protein Cages}

Nature has developed a variety of proteins that function as carriers or storage devices for metal ions and minerals. The iron storage protein ferritin is probably the most intensively studied and best understood example. In this protein, the mineral is sequestered within one single molecule, which has a 
shell of well-defined size and form. Since the early 1990 s, the use of ferritin and other storage proteins as nanometer-sized reaction vessels has been explored. ${ }^{304}$

\subsubsection{Ferritin}

Ferritins are found throughout the animal, plant, and microbial kingdoms. ${ }^{308}$ They consist of a central core of hydrated iron(III) oxide encapsulated within a multisubunit protein shell. Ferritins are robust proteins, which can withstand high temperatures (85 ${ }^{\circ} \mathrm{C}$ ) and high $\mathrm{pH}$ 's (8.5-9) for an appreciable period of time without significant disruption of their quaternary structure. Iron-free ferritin molecules (apoferritin) are composed of 24 polypeptide subunits, which assemble into a hollow sphere of ca. $500 \mathrm{KDa}$. The outer diameter of the protein is $12 \mathrm{~nm}$, while the inner diameter is $8 \mathrm{~nm}$. The ferritin protein shell has several functions: it acquires iron(II), catalyses its oxidation, and induces mineralization within its cavity. Up to 4500 iron atoms can be housed within the protein cavity. Such a high iron-to-protein ratio is made possible by sequestering the iron as a compact mineral, which has a structure similar to that of the mineral ferrihydrite $\left(5 \mathrm{Fe}_{2} \mathrm{O}_{3} \cdot 9 \mathrm{H}_{2} \mathrm{O}\right)$. Hydrophilic and hydrophobic channels penetrate the shell, and, as a result, iron atoms can be removed from the cage by reductive dissolution. In vitro reconstitution of iron(III) oxide cores can be readily achieved by room-temperature incubation of intact empty protein (apoferritin) with $\mathrm{Fe}(\mathrm{II})$ solutions at moderate $\mathrm{pH}^{309}$

In 1991, Mann and co-workers began to explore the use of Ferritin as a nanometer-sized bioreactor for producing monodisperse metal particles from metal ions other than the natural hydrated iron(III) oxide. They showed for the first time that supramolecular protein cages have the potential to act as constrained reaction environments in the synthesis of inorganic materials of nanometer dimensions. Horse spleen apoferritin (HSFn) was used as a nanocontainer to generate iron sulfide particles, and manganese oxide and uranyl oxohydroxide crystals. ${ }^{309,310}$ In Figure 33, the three different approaches followed by Mann and co-workers are schematically depicted. For example, incubation of the empty protein cages with $\mathrm{MnCl}_{2}$ at $\mathrm{pH} 9$ resulted in specific manganese ion uptake, oxidation, and precipitation. Depending on the original concentrations of protein and $\mathrm{Mn}$ (II), discrete manganese oxide cores up to $7 \mathrm{~nm}$ in diameter were formed, as was shown by TEM and the electron dispersive X-ray analysis (EDXA) spectrum. Further studies were carried out on the synthesis of iron sulfide, ${ }^{311}$ manganese oxide, ${ }^{312}$ and cobalt oxyhydroxide, ${ }^{313}$ within the nanosized cavity of horse spleen ferritin.

Later work consisted of synthesizing the ferromagnetic nanocrystals of magnetite $\left(\mathrm{Fe}_{2} \mathrm{O}_{4}\right),{ }^{314}$ and magnetite/maghemite $\left(\mathrm{Fe}_{3} \mathrm{O}_{4} / \gamma-\mathrm{Fe}_{2} \mathrm{O}_{3}\right),{ }^{315}$ within the ferritin cavity to construct a magnetic protein, "magnetoferritin". As a result of the restricted space within the nanoreactors, crystalline inorganic particles with diameters of ca. 6-7 nm were formed, as determined by TEM. The magnetite/maghemite particles were
A
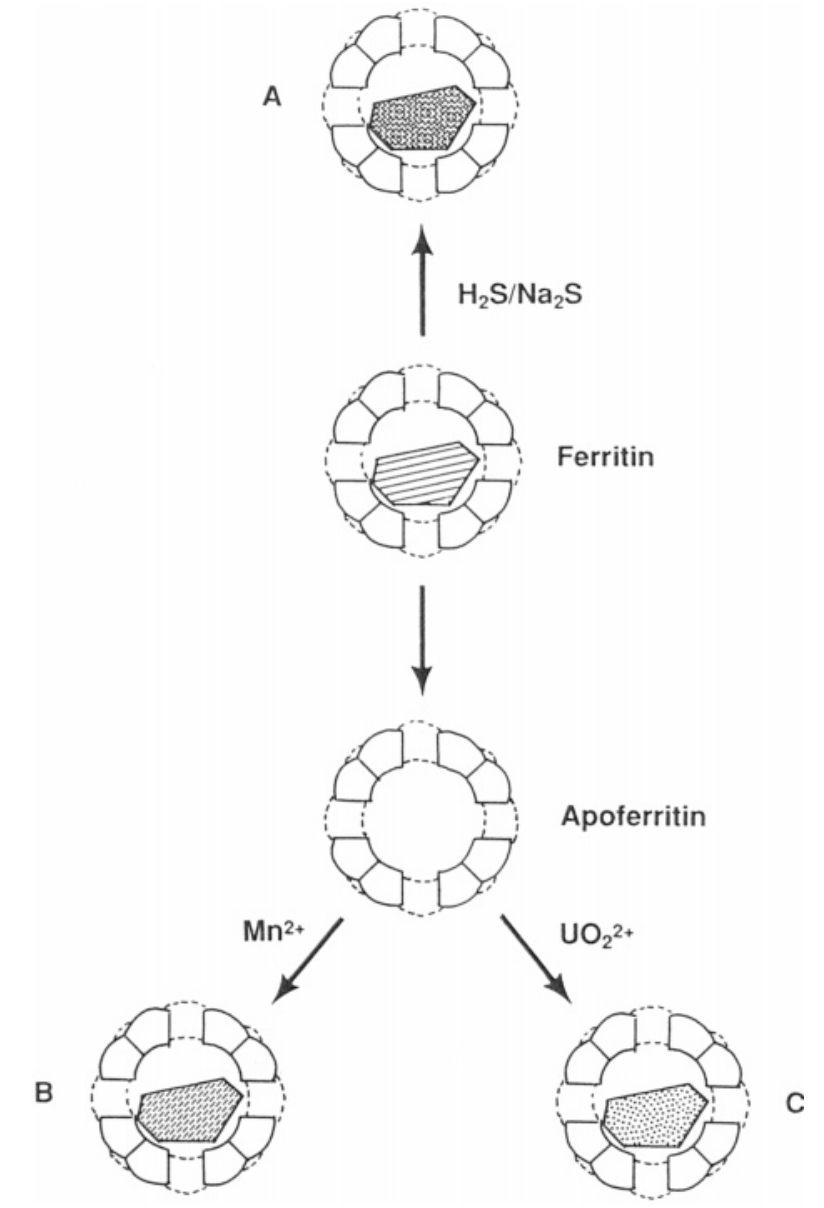

Figure 33. Schematic representation of the use of ferritin in the synthesis of nanophase materials. (a) Iron sulfide formation by in situ reaction of native iron oxide cores. (b) Manganese oxide reconstitution by redox-driven reactions within apoferritin. (c) Uranyl oxy-hydroxide deposition by ion-binding and hydrolytic polymerization. (Reproduced with permission from ref 310. Copyright 1991 Wiley.)

generated by trimethylamino- $N$-oxide oxidation of apoferritin, which was loaded with various amounts of iron(II) ions. The temperature-dependent magnetic properties of magnetoferritins with different iron loadings were studied by SQUID magnetometry.

A new approach was introduced by Douglas et al., who studied the protein-constrained iron oxide core of ferritin as a catalyst for photoreduction reactions. They demonstrated that the iron oxide nanoparticle encapsulated within the protein cage of ferritin can act as a semiconductor photocatalyst for the reduction of $\mathrm{Cr}(\mathrm{VI})$ to $\mathrm{Cr}$ (III). ${ }^{316}$ Furthermore, the same system was shown to be capable of photoreducing $\mathrm{Cu}-$ (II) to form a colloidal dispersion of $\mathrm{Cu}(0)$ with a fairly narrow size distribution. ${ }^{317}$ Examination of the photolysis products by TEM revealed electron-dense spheroid particles. It was found that the molar ratio of $\mathrm{Cu}(\mathrm{II}) /$ ferritin was the major factor in determining the particle size. A similar approach was followed recently by $\mathrm{T}$. Ueno and co-workers when they constructed a size-selective hydrogenation biocatalyst. They encapsulated a $\mathrm{Pd}$ nanocluster in the apoferritin cavity by in situ chemical reduction of $\mathrm{Pd}$ (II) ions. Subsequently, the catalytic hydrogenation of olefins by the $\mathrm{Pd}$-apoferritin hybrid was evaluated in aqueous media. It was shown that the catalytic 

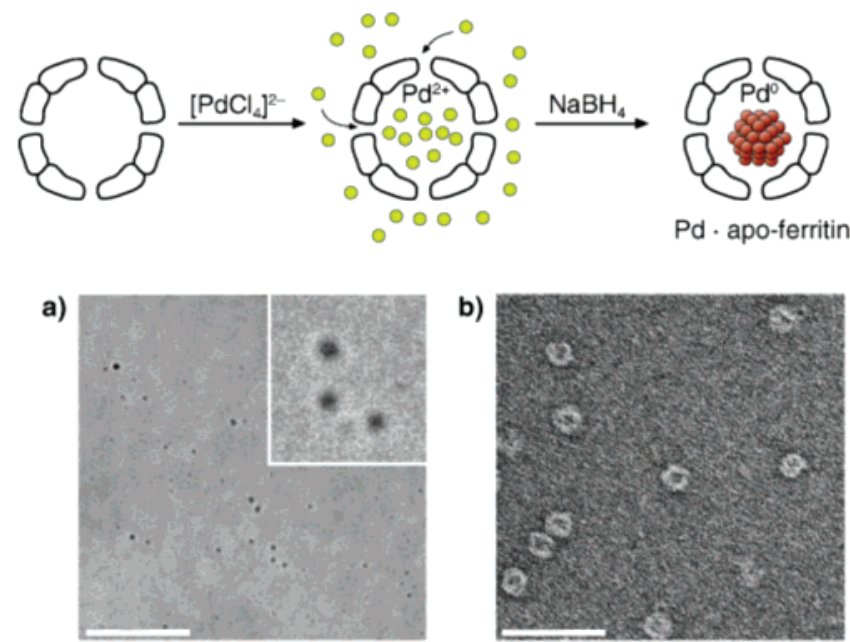

Figure 34. Top: Scheme showing the preparation of Pdapoferritin. Bottom: TEM images of the Pd-apoferritin, (a) an ice-embedded unstained sample (inset magnification $4 \times$ ), (b) a sample negatively stained with uranyl acetate. Scale bars represent $50 \mathrm{~nm}$. (Reproduced with permission from ref 318. Copyright 2004 Wiley.)

system discriminates with respect to size of the substrates, via the 3 -fold channels that cross the protein cage (Figure 34 ). ${ }^{318}$

Alteration of the outer surface of ferritin has been investigated by Mann and co-workers. Alkylated derivatives of ferritin were prepared by carbodiimideactivated coupling of long chain primary amines to carboxylic acid residues on the protein surface. ${ }^{315,319}$ As a consequence of this surface modification, the proteins become soluble in several organic solvents as intact, nonaggregated biomolecules. The long-term goal of this approach is to exploit alkylated ferritins as nanoreactors for condensation reactions involving metal alkoxides and related organometallic compounds, which is only possible in nonaqueous systems. Another surface modification involved the preparation of biotinylated ferritin. In this case, the outer protein surface was derivatized with approximately 70 biotinylated ligands that were available to interact with streptavidin to form a cross-linked network of biomacromolecules. Such ferritin-based arrays could have important applications in magnetic storage and nanoelectronic devices. ${ }^{320}$

An unusual form of ferritin was discovered in the Gram-positive bacterium Listeria innocua. ${ }^{321}$ This ferritin-like protein was also utilized as a size and shape constrained reaction environment, as previously described for horse spleen ferritin. L. innocua ferritin differs from other known ferritins in that its protein shell is assembled from 12 identical subunits; it can accumulate only 500 iron atoms within its 5 $\mathrm{nm}$ diameter central cavity. Ferrimagnetic iron oxide nanoparticles, ${ }^{322}$ and cobalt oxide minerals, ${ }^{323}$ were synthesized within the protein cage. Size exclusion chromatography (SEC), dynamic light scattering (DLS), gel electrophoresis, and TEM are the techniques that were used to characterize the mineralized protein cages.

\subsubsection{Other Protein Cages}

The investigations on ferritin as a size and shape constrained reaction vessel have paved the way for other groups to use different biomolecular systems to develop a general class of self-assembled bioinorganic hybrid materials with designed functionality and biocompatibility. Apart from ferritin, other proteins (see below) and even virus cages (see section 4.2) have been used as bionanoreactors.

Lumazine synthase is a hollow icosahedral bacterial enzyme of $1 \mathrm{MDa}$ molecular weight, which is formed by self-assembly of 60 subunits. ${ }^{324}$ The selfassembled structure of this protein is very similar to those of the protein cages described above. Following the same concept as published for the protein ferritin, lumazine synthase was shown to be a suitable mineralization template for the fabrication of nanocrystalline iron oxide.

The small heat shock protein cage from Methanococcus jannaschii (MjHsp) was shown to be a versatile platform for genetic and chemical modifications. ${ }^{325}$ This protein consists of 24 subunits which self-assemble to form a cage with octahedral symmetry. The protein has an exterior diameter of 12 $\mathrm{nm}$; it is stable in the $\mathrm{pH}$ range $5-11$ and can withstand temperatures up to $\sim 70{ }^{\circ} \mathrm{C}$. The possibility to functionalize this template with organic and inorganic groups was recently studied by Douglas and co-workers. Thiol groups, obtained by modification of the protein, and endogenous amine groups on both the exterior and the interior surfaces, were reacted with activated fluorescein. The covalent attachment of fluorescein to the protein cages was demonstrated by SEC, DLS, TEM, and SDS polyacrylamide gel electrophoresis (PAGE). Furthermore, by analogy to ferritin, $\mathrm{MjHsp}$ was investigated for its ability to act as a size constrained reaction environment for iron oxide mineralization. Both the modified and the native MjHsp cages showed similar mineralization capabilities. Small iron oxide particles of $\sim 9 \mathrm{~nm}$ were observed when the mineralized protein was imaged by TEM.

\subsection{Viruses}

Virus particles typically consist of several hundred to thousands of protein molecules that self-assemble to form a hollow scaffold, which holds the viral nucleic acid. In the past decade, there has been a growing interest in the use of virus particles as templates for nanosized materials. Viral capsids (the self-assembled protein cage structures) offer robust and monodisperse structures and exist in a large variety of sizes and shapes. Moreover, the protein surface can be used as a synthetic platform for chemical modification. Because certain types of viruses can be obtained in large quantities and manipulated at the genetic level, they afford a unique opportunity for chemists to expand the repertoire of natural starting materials for synthetic and catalytic applications (Figure 35).

\subsubsection{Rod-Shaped Viruses}

Tobacco Mosaic Virus. The Tobacco Mosaic Virus (TMV) is the most comprehensively understood system of biological self-assembly to date. TMV is a stable virion that can withstand temperatures of up to $60{ }^{\circ} \mathrm{C}$ and $\mathrm{pH}$ values as low as 2 and as high as 


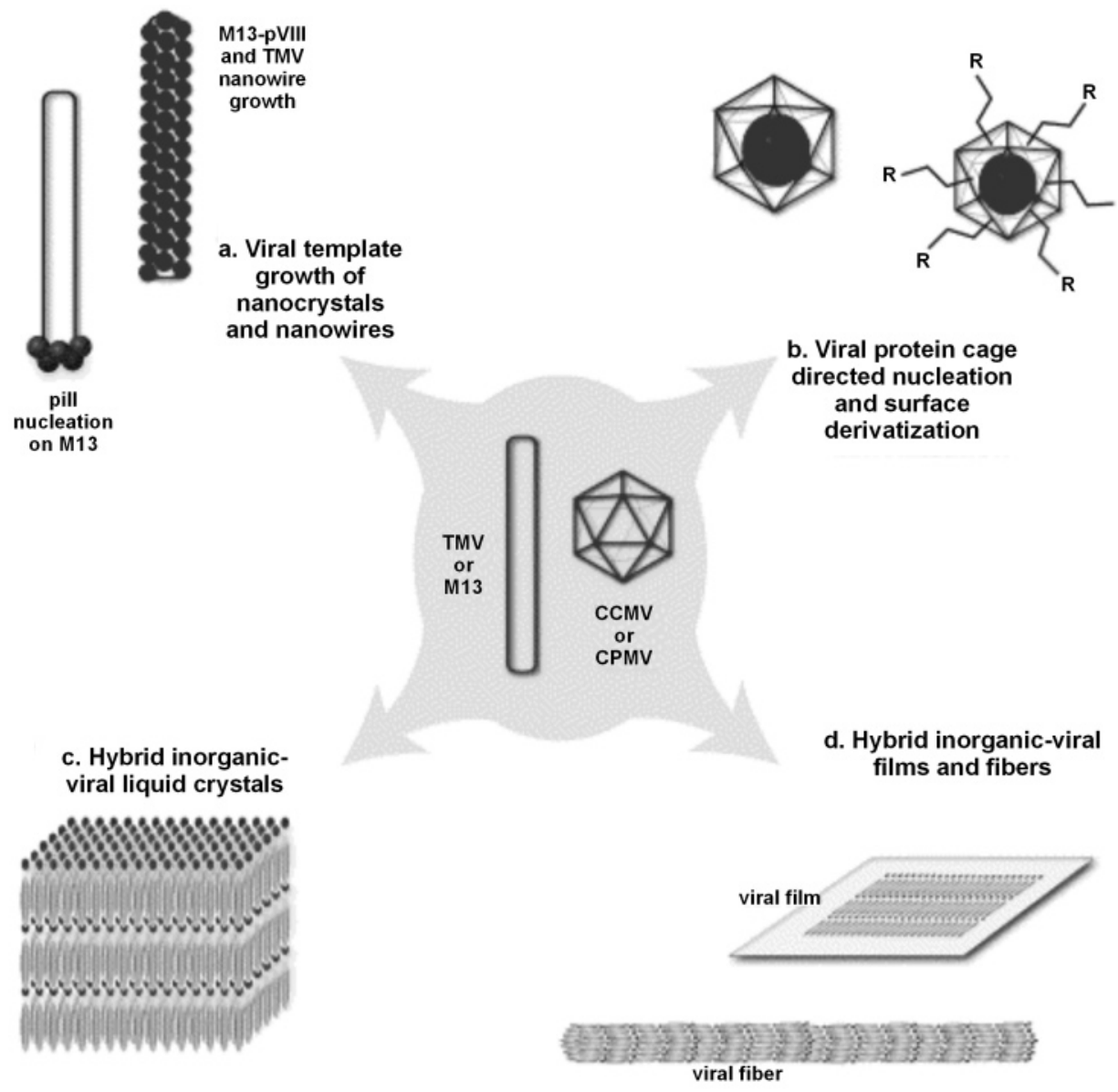

Figure 35. Some of the routes followed to synthesize biohybrid materials using rod-shaped (TMV, M13) and cage-structured (CCMV, CPMV) viruses. (Reproduced with permission from ref 305. Copyright 2003 Elsevier.)

10. It is made up of 2130 identical protein building blocks, arranged in a helical motif around the viral RNA to form a hollow tube of $300 \times 18 \mathrm{~nm}$ in size with a central channel that has a diameter of $4 \mathrm{~nm} .{ }^{304}$

The internal and external surfaces of the TMV protein capsid consist of repeat patterns of charged amino acid residues such as glutamate, aspartate, arginine, and lysine. Mann and co-workers have used these functionalities as nucleation sites for surfacecontrolled biomineralization. The surface chemistry of TMV coupled with the high stability of the protein assembly provides a structural framework for the site-specific nucleation of a variety of inorganic solids (Scheme 13). Accordingly, the template-directed synthesis of organic-inorganic nanotubes was performed. ${ }^{326}$ The electrochemical modification of the TMV inner and outer surface was performed by Knez et al., via electroless deposition of different metals such as nickel and silver. Depending on the metallization conditions, they could selectively target the outer or inner surface of the tubelike TMV, as was shown by TEM. ${ }^{327}$ Furthermore, the same group used TMV for the synthesis of metal nanowires. The central channel of the virus was used as a template to synthesize nickel and cobalt wires of only a few atoms in diameter and lengths up to the micrometer range. ${ }^{328}$

Motivated by the fact that 1D, nanometer-sized objects are needed for applications in nanoelectronics and nanobiotechnology, Dujardin et al. investigated
Scheme 13. Routes for the Synthesis of Nanotube Composites Using TMV Templates ${ }^{a}$
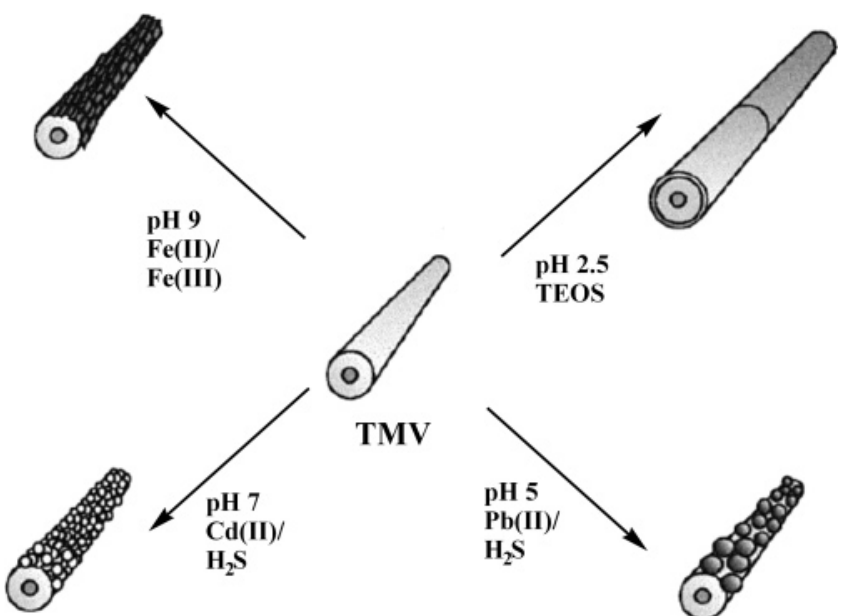

a Clockwise from top right: sol-gel condensation (silica); coprecipitation ( $\mathrm{PbS}$ and $\mathrm{CdS}$ nanocrystals); oxidative hydrolysis (iron oxide). (Reproduced with permission from ref 326. Copyright 1999 Wiley.)

the use of cylindrical particles of TMV as template for the in situ formation of anisotropic assemblies of spherical $\mathrm{Pt}, \mathrm{Au}$, or Ag nanoparticles. By chemically controlling the surface charge of the virus, metallization could selectively be performed on the outer or inner surface of the protein capsid (Figure 36). ${ }^{329}$ Tubular structures using TMV as a template were 


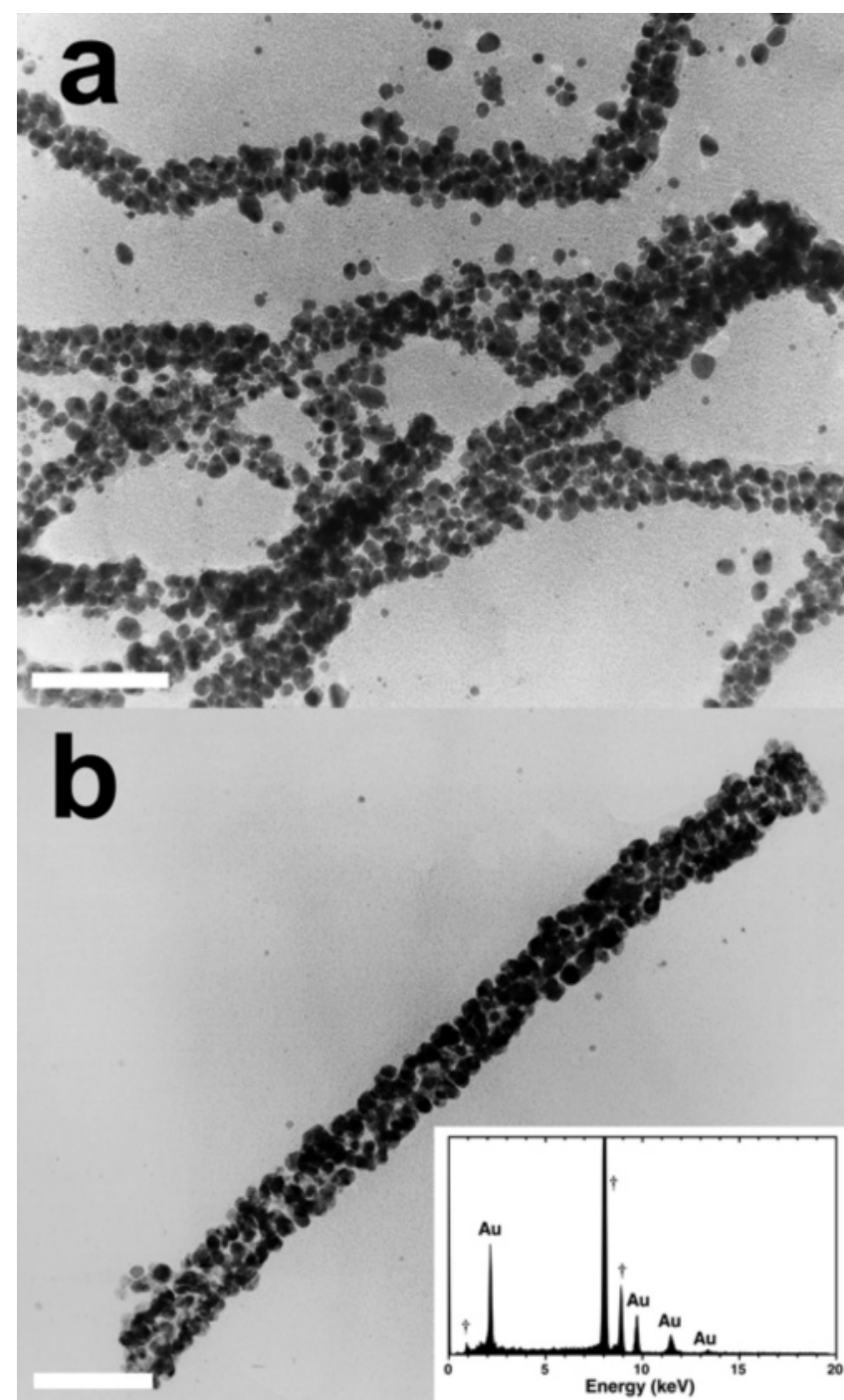

Figure 36. TEM micrographs of gold nanoparticles produced in the presence of wild-type TMV. (a) Low magnification image showing multiple TMV rods with dense external coating of gold nanoparticles; scale bar $=100 \mathrm{~nm}$. (b) Single TMV rod with dense coating of discrete gold nanoparticles; scale bar $=50 \mathrm{~nm}$. Inset: EDXA spectrum showing $\mathrm{Au}$ and $\mathrm{Cu}(\dagger)$ (from supporting grid). (Reproduced with permission from ref 329. Copyright 2003 American Chemical Society.)

also obtained by Fujikawa et al. They assembled TMV on a surface and then covered it with ultrathin titania films by the surface sol-gel process. ${ }^{330}$

Aggregates of TMV have been thoroughly studied as well. The formation of ordered aggregates of TMV in the presence of divalent metal ions was studied in concentrated solutions of the virus. Some divalent metal cations were found to promote TMV precipitation, and nematic liquid crystalline behavior was observed for the precipitates. ${ }^{331}$ Nematic liquid crystals of TMV have been used to prepare silica nanoand mesostructures with parallel or radial arrays of linear channels, respectively. ${ }^{332}$ Recently, the adsorption behavior and surface behavior of TMV on different surfaces was studied. The resulting virion on the surface was imaged by noncontact AFM. These experiments provided information on how to image the TMV and also revealed that TMV keeps its cylindrical structure only under conditions of weak absorption, that is, on hydrophobic surfaces. AFM experiments showed that it is possible to image TMV at solid-air and solid-liquid interfaces on welldefined substrates such as mica and $\mathrm{Au}(111){ }^{333}$

M13 Bacteriophage. Another example of the use of viruses for the assembly of inorganic materials has been given by Belcher and co-workers. They were inspired by living organisms that have the ability to nucleate and assemble inorganic materials with near perfect alignment, orientation, and shape to form organic-inorganic hybrid structures, such as shells and bones. The group has used the M13 bacteriophage as a template for the nucleation and growth of nanocrystals and the formation of inorganic tubes and wires. They have shown, furthermore, that these inorganic-virus hybrids can self-assemble into films, fibers, and liquid crystalline materials. Their research (until 2003) has been summarized in a review, ${ }^{305}$ together with some other studies, in which viruses are used as scaffolds for the growth of minerals and the assembly of materials.

The M13 bacteriophage is a rod-shaped virus, which infects bacteria. This virus contains a singlestranded DNA and three different types of coat proteins: $p$ VIII, $p$ III, and $p$ IX. A precise number (2700) of copies of the major coat protein, p VIII, are arranged in a repeating helical array, forming a flexible cylinder in which the DNA is encased. Five copies of the two minor coat proteins, p III and pIX, are situated at each end of the virus. The wild-type virus is approximately $860 \mathrm{~nm}$ long and $6.5 \mathrm{~nm}$ wide. The viral coat proteins can be easily modified by genetic engineering to insert nucleation centers on the virus surface or to specifically functionalize viruses with different binding motifs. ${ }^{334}$

Genetically engineered M13 bacteriophage viruses were used to find virus surface peptides that specifically recognize crystalline semiconductor materials. To this end, combinatorial libraries of the virus containing $\sim 10^{9}$ different random peptide sequences fused to the coat protein were made. Some peptide sequences isolated from the library showed very high affinities toward $\mathrm{ZnS}$ and CdS. ${ }^{305,334,335}$

In a subsequent series of experiments, it was verified that the peptides found through selection can be used to template and grow materials, in a way analogous to that of abalone shells in nature. Two peptide sequences that were selective for $\mathrm{ZnS}$ and CdS were shown to control nanocrystal particle size and shape. It was also found that a difference in peptide structure (cyclic versus linear) led to the nucleation of different crystal phases in both the $\mathrm{ZnS}$ and the CdS systems. These experiments show that the M13 virus allows for the genetic control of semiconducting, metallic, metal oxide, or magnetic material using the same viral template. Furthermore, the possibility to control the size and phase of the material opens the way to tune their properties and could be a first step toward a next generation of microelectronic devices using "biological" materials. ${ }^{305,334,336}$ The same concept has been used recently to prepare ferromagnetic materials. The M13 bacteriophage has been engineered to template the growth of FePt nanoparticles. EM and X-ray studies indi- 


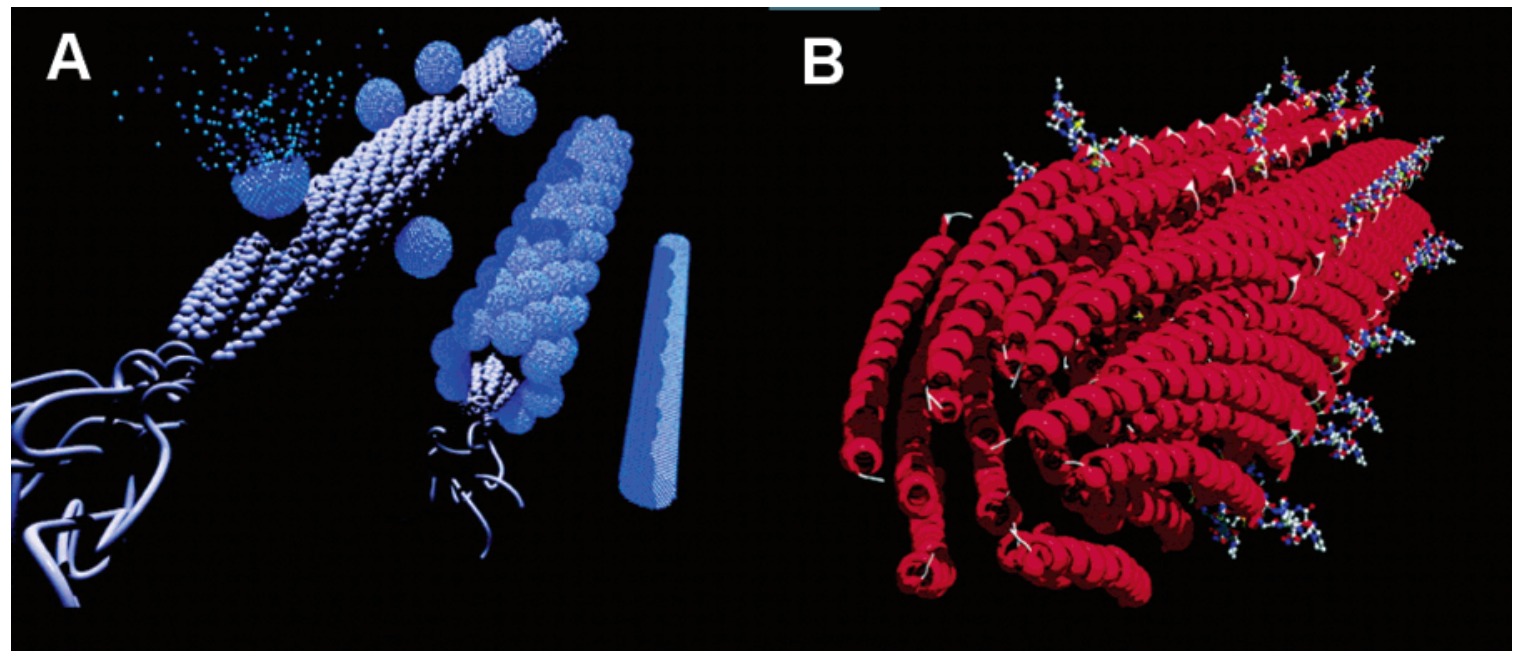

Figure 37. Nanowire synthesis using the M13 bacteriophage as a template. (a) The general scheme of the nanowire synthesis. (b) Picture showing the packing of the expressed peptides, which are responsible for the ordered nucleation of the nanocrystal particles. (Reproduced with permission from ref 307. Copyright 2004 American Association for the Advancement of Science.)
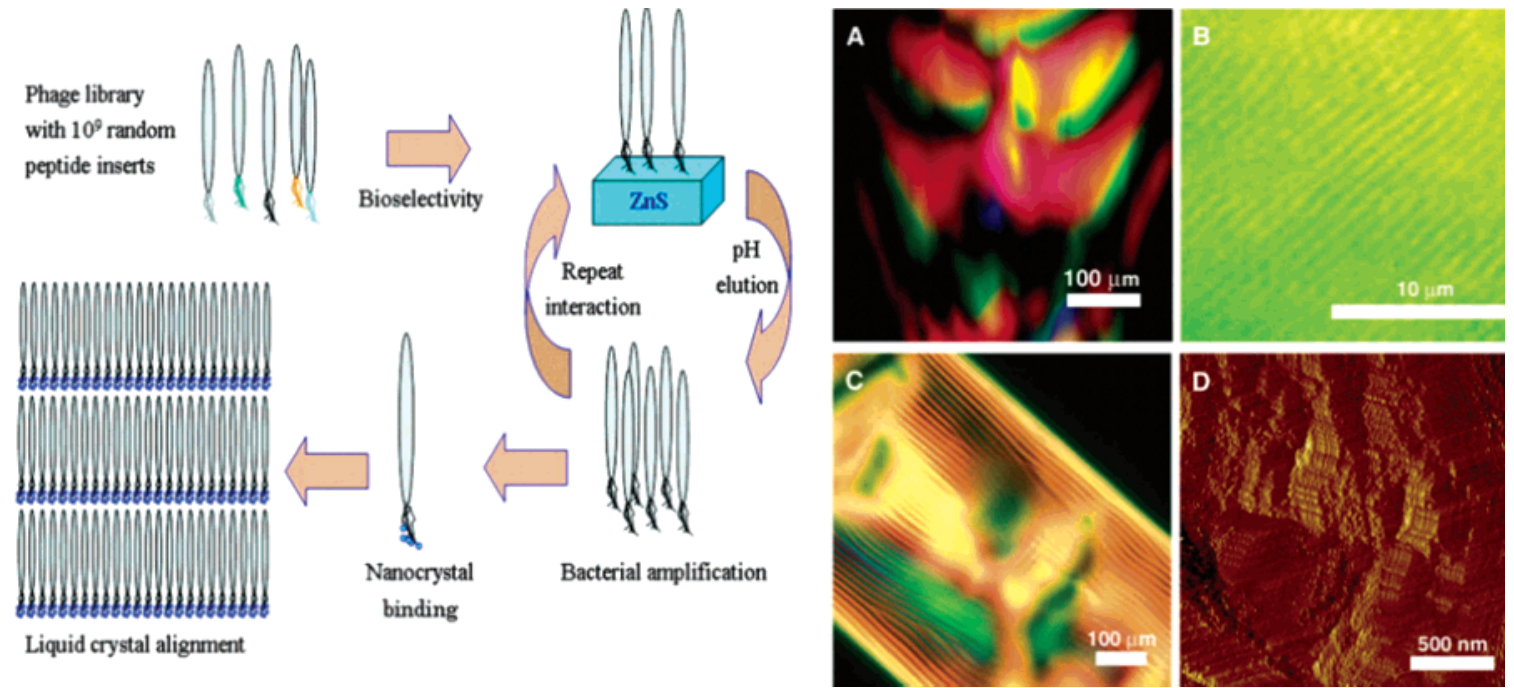

Figure 38. Left: Schematic diagram of the one-step process used to generate liquid crystalline films from A7 phage-ZnS hybrid particles by the phage display method. Right: Characterization of the liquid crystalline suspensions and cast film. (Reproduced with permission from ref 336. Copyright 2002 American Association for the Advancement of Science.)

cated that the particles were composed of an $\mathrm{FePt}$ alloy with some degree of chemical ordering. ${ }^{337}$

In another study, M13 viruses have been applied as templates for the synthesis of monodisperse, crystalline nanowires of different compositions. First, the genetically modified M13 virus scaffold was used to assemble crystalline nanoparticles into a 1D array. This was followed by a mineralization process, and finally the mineralized virus was annealed, allowing the polycrystalline assembly to form a single-crystal nanowire through the removal of the organic template (Figure 37). ${ }^{305,307,338}$

To bind other compounds to the M13 virus, this biomolecule was functionalized on one end with a specific binding moiety for streptavidin. ${ }^{335}$ Antistreptavidin M13 virus is the name of the virus that contains this specific peptide able to bind streptavidin. Gold nanocrystals, fluorescein, and phycoerythrin were conjugated with streptavidin and subsequently mixed with the anti-streptavidin M13. Highly concentrated mixtures gave suspensions which showed liquid-crystalline properties. ${ }^{339}$ In another paper, 1D metal ring structures based on genetically engineered M13 virus and on anti-streptavidin viruses have been described. ${ }^{340}$ These approaches open a way to the alignment of nanosized materials by conjugating them to streptavidin.

Genetically engineered viruses have been used to self-assemble nanomaterials into liquid crystals, films and fibers. ${ }^{305}$ Their ordered structures and morphologies have been studied by polarized optical microscopy (POM), atomic force microscopy (AFM), and EM. These viral-inorganic hybrid materials, organized into liquid crystalline films or fibers, show great potential for the construction of tunable devices. They are made by a one-pot synthesis route: high concentrations of genetically engineered viruses are suspended in ZnS precursor solutions and directly form viral-ZnS nanocrystal liquid crystalline suspensions (Figure 38). The liquid crystalline behavior of the suspensions is dominated by the long-rod shaped structure of the phage, despite the attached 
a)
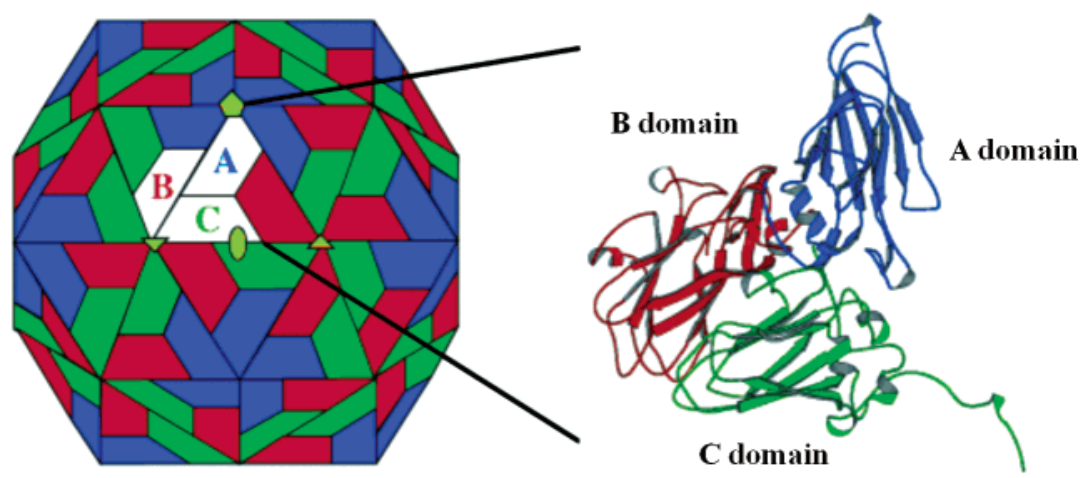

b)

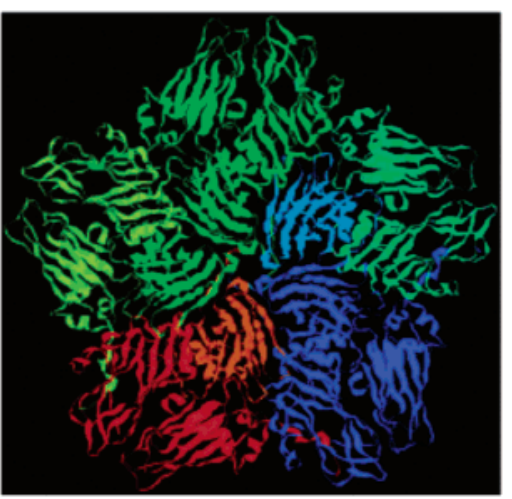

c)

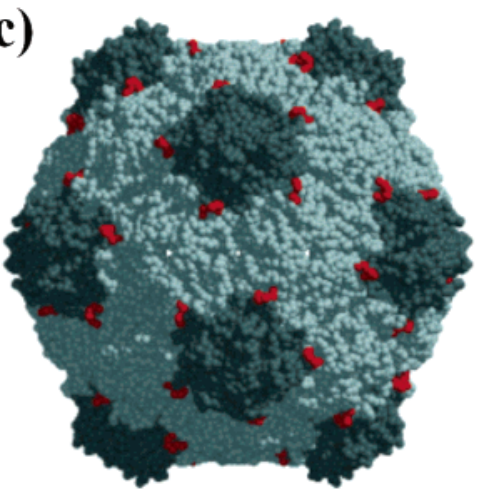

Figure 39. Structure of cowpea mosaic virus and its crystals. (a) Left: Schematic representation of CPMV, showing the distribution of the subunits that comprise the asymmetric unit. Right: The folds of the two subunits, A and B+C. (b) Organization of five asymmetric units into a "pentamer" centered around a small hole at the 5-fold axis. (c) X-ray crystal structure of CPMV highlighting the EF-loop (in red) in the large subunit in which the cysteine-containing insert is made. (Reproduced with permission from ref 343. Copyright 2002 Wiley.)

ZnS nanocrystals. ${ }^{336}$ Long-range ordered, virus-based films have been fabricated using M13 bacteriophage particles, which were aligned and assembled. Their ordered structures and morphologies were studied and characterized, and these viral films were determined to have a chiral smectic C structure. ${ }^{341} \mathrm{M} 13$ virus-based micro- and nanofibers, furthermore, have been fabricated using wet-spinning and electrospinning processes. The resulting fibers showed nematic ordered morphologies due to shear flow. ${ }^{342}$

\subsubsection{Cage-Structured Viruses}

CPMV Virus as a Platform for Organic Chemical Reactions. Finn and Johnson have shown how viruses can function as convenient and programmable platforms for different types of chemical reactions. They used the plant virus Cowpea Mosaic Virus (CPMV), which can be isolated in gram quantities, for this purpose. The virus possesses a structure that is known at atomic resolution, and it is remarkably stable; its particles maintain their integrity at $60{ }^{\circ} \mathrm{C}(\mathrm{pH} 7)$ for at least $1 \mathrm{~h}$ and at room temperature and $\mathrm{pH}$ values from 3.5 to 9 for almost indefinite periods of time. ${ }^{343}$ The virus, therefore, is of potential use as a nanoscale building block in synthesis. The CPMV capsid is formed by 60 copies of an asymmetric unit which contains two protein subunits: a small subunit, the A domain, and a large subunit, the B+C domain (Figure 39). These 60 asymmetric units selfassemble around a single-stranded RNA genome to form the virus particle. The virus displays icosahedral symmetry, its outer diameter being ca. $30 \mathrm{~nm}$. The wild-type CPMV was found to possess per virus particle 60 lysines with enhanced reactivity. Furthermore, four CPMV mutants bearing cysteine residues on the protein surface have been prepared. The chemical reactivity of wild-type CPMV, ${ }^{344}$ and mutant CPMV, ${ }^{345}$ toward lysine- and cysteine-selective reagents, has been thoroughly investigated to demonstrate that CPMV is a versatile, virus-based starting material for chemical synthesis.

Finn and co-workers have shown that organic and inorganic compounds can be attached to the CPMV capsid via the amine and thiol functionalities on the protein surface. ${ }^{344,345}$ They have compared the reactivity of the thiol groups in the wild-type CPMV, which contains cysteine residues only on the interior surface of the virus, with that of CPMV mutants containing thiol groups on the outer surface. ${ }^{343,345}$ Functionalized organic dyes (e.g., fluorescein) that react with the thiol groups were used for this purpose. The derived particles were characterized by SEC FPLC, SDS PAGE, and TEM, and it was concluded that the conversions were quantitative. ${ }^{343-345}$ Furthermore, CPMV and mutant CPMV have been decorated with biotin groups. ${ }^{344,345}$ Adding avidin to these modified virion capsids gave crosslinked networks. These types of experiments provide information as to whether the reactive thiol and amine groups are situated on the inside or outside surface of the virus particle.

Once the reactivity of the different functional groups on the inner and outer surface of the CPMV had been determined, different types of modifications on the virus could be performed, for example, the construction of hybrid CPMV virus-polymer par- 
Scheme 14. Preparation of Virus-Stilbene-PEG Conjugates ${ }^{a}$

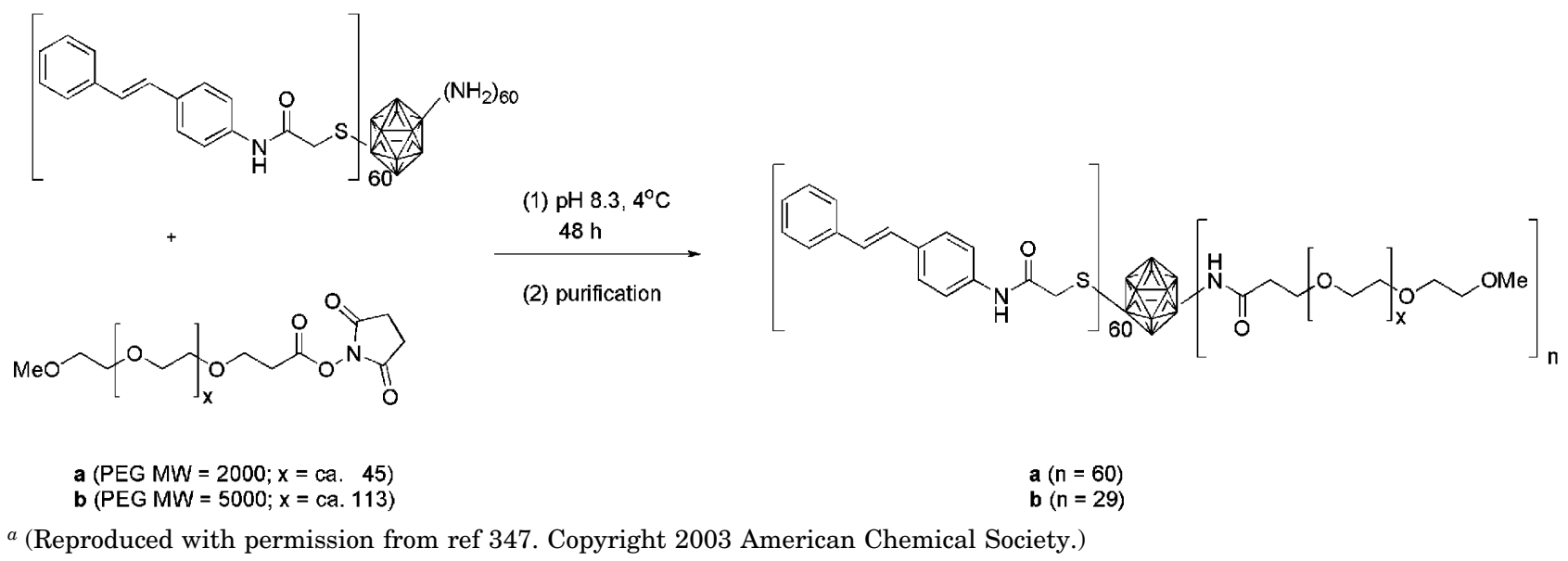

ticles by derivatizing the outer surface of the virus with poly(ethylene glycol), PEG. ${ }^{346}$ The resulting hybrids had physical and immunogenic properties that were markedly different from those of the native virus. Furthermore, the PEGylation reaction could be used to block the binding of a blue fluorescent antibody to its antigen (stilbene), which was also bound to the cysteine residues on the CPMV surface (Scheme 14). ${ }^{347}$

The exterior surface of the protein cage of CPMV has also been decorated with azide and alkyne groups, which were reacted with fluorescein derivatives containing the complementary groups for a copper(I)-catalyzed azide-alkyne [3+2] cycloaddition reaction ("Click" reaction). ${ }^{348}$ Additionally, CPMV particles have been functionalized with carbohydrate molecules to study the binding of the virus to carbohydrate-binding proteins and cells. ${ }^{349}$ Furthermore, different mutants of CPMV have been used as scaffolds to bind gold nanoparticles through goldsulfur bond formation. Unstained TEM images showed patterns that were in line with the locations of the cysteine functions on the capsid. By using the CPMV capsid as a template, and the monodispersity and chemical specificity of the biological system as an additional feature, gold nanoparticles could be assembled into complex 3D. ${ }^{350}$ Very recently, it was shown how the use of the reagent Ni/GGH/MMPP induces cross-linking between tyrosine residues in adjacent subunits of the CPMV virus and, furthermore, generate tyrosyl radicals at other positions of the virus, which are amenable to trapping with external disulfide reagents. ${ }^{351}$ Additionally, complementary 20-mer oligonucleotides were attached recently to CPMV scaffolds either at lysine residues or at genetically engineered cysteines to study their assembly behavior. ${ }^{352}$

Different approaches have been followed to assemble CPMV particles on surfaces. Complex patterns could be made by allowing concentrated CPMV droplets to dry on mica surfaces. These patterns have been studied by AFM. ${ }^{353}$ Furthermore, dip pen nanolithography (DPN) has been combined with chemoselective protein-to-surface linkers to create nanometric chemical templates for the fabrication of arrays of virus particles. To this end, DPN was used to pattern a gold substrate with a thiol-selective linker after which a genetically modified Cys-CPMV was immobilized. . $^{354,355}$

Bacteriophage MS2. The bacteriophage MS2 is a virus with a structure similar to that of CPMV. The capsid shell of bacteriophage MS2 comprises 180 sequence-identical protein monomers assembled into an icosahedral arrangement that is $27 \mathrm{~nm}$ in outer diameter. A large number of pores $(32,1.8 \mathrm{~nm}$ in diameter) are present in the capsid, providing access to the interior of the virus.

Francis and co-workers have followed the approach of Finn and Johnson, ${ }^{345}$ and Douglas and Young, ${ }^{325,356}$ to modify and decorate the protein surface of the MS2 virus (Figure 40). It involves the covalent modification of the interior surface of the viral capsid with the objective of constructing targeted drug delivery systems. Tyrosine residues were modified to obtain a functional handle for a Diels-Alder bioconjugation reaction. 357

Host-Guest Properties of Viruses. The welldefined Cowpea Chlorotic Mottle Virus (CCMV) has been used as a nanoreaction vessel for the reversibly gated entrapment of organic and inorganic compounds. CCMV virions are $28 \mathrm{~nm}$ in diameter, and the protein shell defines an inner cavity of approximately $18 \mathrm{~nm}$. This virus is composed of 180 identical coat protein subunits which encapsulate the RNA. ${ }^{358}$

One interesting feature of the CCMV is its sensitivity to $\mathrm{pH}$ and ionic strength. ${ }^{359}$ Depending on the $\mathrm{pH}, \mathrm{CCMV}$ virions can be rapidly disassembled in vitro to dimers and reassembled again. After removal of the RNA, purified viral coat protein subunits can be easily assembled in vitro into empty virion particles. The cavity is approximately twice the diameter of the iron storage protein ferritin, and structural analysis has demonstrated that the highly basic $N$-termini (arginine and lysine residues) of the 180 subunits project into the interior of the protein cage. Even though these basic amino acid residues are required to package the viral RNA, genetic analysis has shown that they are not required for the selfassembly of the empty protein cage in vitro.

Dynamic structural transitions occur in many virions, often induced by specific chemical signals. They provide unique molecular gating mechanisms to control the containment and release of entrapped 


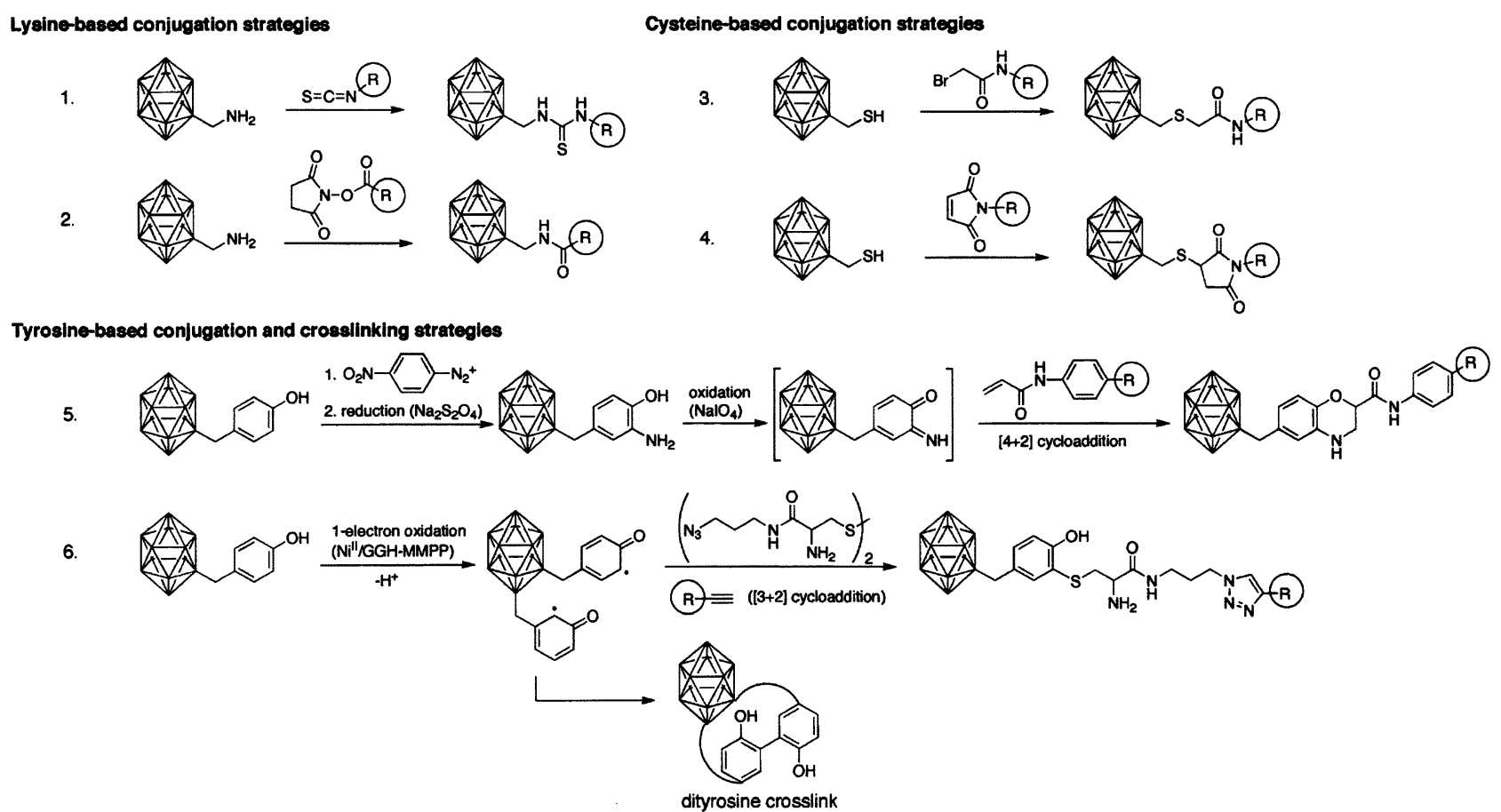

Figure 40. Strategies for the specific chemical modification of viral particles to generate virus-based scaffolds for nanomaterials. (Reproduced with permission from ref 306. Copyright 2004 Elsevier.)

material. CCMV undergoes a reversible pH-dependent swelling, which results in a $10 \%$ increase in virus dimensions. Structural analysis has revealed that CCMV swelling leads to the formation of 60 separate openings (2 $\mathrm{nm}$ in diameter). Douglas and Young have used the $\mathrm{pH}$-dependent gating of the CCMV to control the entrapment of different guest compounds.

Inspired by ferritin, CCMV has been used as a host system for the encapsulation of guest compounds that have a complementary size and shape. ${ }^{303,360}$ The host-guest relationship between the viral cage and the encapsulated material is primarily based on a complementary electrostatic interaction. In the native viral protein cage, the cationic interior of the virus interacts with the polyanion of RNA. This electrostatic host-guest interaction can be used to bind other (poly)anions.

Douglas and Young have reported on the encapsulation of two polyoxometalate species (paratungstate and decavanadate) and an anionic polymer inside the CCMV cage, controlled by the $\mathrm{pH}$-dependent gating of the virion's pores. For example, empty virions were incubated with inorganic molecular tungstate $\left(\mathrm{WO}_{4}{ }^{2+}\right)$ at $\mathrm{pH}$ 6.5, concentrated, and subsequently washed at $\mathrm{pH} 5$, at which point the pores in the protein shell closed and the tungstate ions oligomerized (Figure 41). The latter process is a crystallization that yielded uniform nanocrystals with a diameter of $6.7 \mathrm{~nm}$, which were characterized by TEM.

To further investigate the electrostatic aspects of this host-guest interaction and the influence of gating on the entrapment, the authors studied the $\mathrm{pH}$-dependent encapsulation of an anionic organic polymer. The polymer was incubated with empty virions at $\mathrm{pH} 7.5$, after which the $\mathrm{pH}$ was lowered to well below the gating threshold $(\mathrm{pH} 4.5)$, resulting

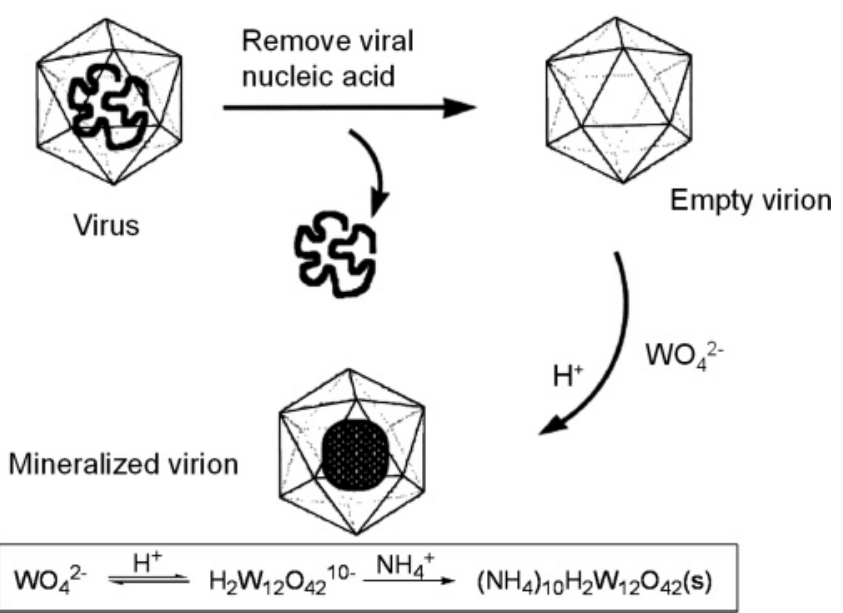

Figure 41. Schematic illustration of the approach used to achieve mineralization within the CCMV virus particle. (Reproduced with permission from ref 360. Copyright 1998 Nature.)

in the selective encapsulation of the polymer. Evidence for this encapsulation came from TEM experiments, which showed that no staining dyes were included in the interior of the virus particles.

The electrostatic properties of the CCMV protein cage interior surface could be altered by replacing nine basic residues at the $\mathrm{N}$-terminus of every protein subunit with glutamic acid residues. The mutant CCMV was formed to assemble readily into a cagelike architecture similar to that of the wild-type. The modified protein cages were treated with Fe(II) salts at $\mathrm{pH} 6.5$ and allowed to oxidize in air, yielding iron oxide cores as was shown by EM. ${ }^{361}$ Genetic modification of the exterior surface of CCMV has also been reported, the objective being to form self-assembled arrays of CCMV viral cages on gold surfaces. ${ }^{362}$ 


\section{Conclusions and Outlook}

Nature has through evolution encased within a cell the machinery for life. The cell as the ultimate nanofactory contains the codes for the synthesis and production of tailored products. Despite our limited understanding of its complex metabolite pathways, cascade reaction conveyer belts, industry has readily adopted, and adapted, cell cultures, which can be envisaged as extremely small "black boxes", to produce numerous products on a large scale. Although the ultimate goal of numerous scientists is to mimic the complexity of cells and construct hybrids of fully synthetic cells, it will take many years before such a goal will be achieved.

In the simplest mimic of a cell, that is, a single type of reaction within an enclosed environment, science has, however, made significant inroads. Enhanced by the wide variety of building blocks available, many novel approaches toward nanoreactors have been achieved. In chronological order, the first approach was the use of simple surfactants for the construction of micellar or emulsion reactions. Although extremely simple in concept, such systems contain many aspects found in natural cells, such as self-assembly and compartmentalization. In the second half of the 20th century, the application of lipid-based vesicular assemblies as potential cell membrane mimics and subsequently as enzyme and catalyst-containing nanoreactors received considerable attention, which resulted in significant, but somewhat limited, success. The advent of supramolecular chemistry and rapidly thereafter block copolymer chemistry, however, suddenly opened up numerous new avenues of research. The ability to program molecules to selfassemble into specific encapsulating architectures and the tentative first catalytic reactions into this emerging field look promising, but as yet has not reached full potential. The application of macromolecular amphiphiles to construct unique vesicle reactors or drug-delivery systems has by contrast expanded rapidly, resulting to date in numerous commercial applications, with many more to come.

The use of more natural building blocks such as enzyme-based giant amphiphiles and virus capsids moves more closely back to the concept of modeling the natural systems. These latter approaches, especially virus capsules, hold many unique advantages, in particular, the ease with which large quantities of monodisperse biocompatible capsules can be obtained.

Overall, the future for nanovessels holds great promise, in particular due to the wide variety of foreseeable applications as reactors, delivery devices, or even components in quantum electronics. The holy grail of reactor synthesis, however, still remains the construction of a synthetic cell. Although such a goal may never be attainable due to the extreme complexity of nature, there is little doubt that many large steps toward such a lofty dream will be achieved, which in turn will lead to unique nanoreactors applicable in industrial processes. The evolution of chemistry is still ongoing.

\section{Acknowledgment}

We thank The Netherlands Organization for Scientific Research (NWO) for financial support of J.A.A.W.E. and J.J.L.M.C. (VENI Grants), and A.E.R. (VIDI Grant).

\section{References}

(1) Pauling, L. Chem. Eng. News 1946, 24, 1375.

(2) Motherwell, W. B.; Bingham, M. J.; Six, Y. Tetrahedron 2001 57, 4663 and references therein.

(3) Gorenstein, D. G. Chem. Rev. 1987, 87, 1047.

(4) Kirby, A. J. Angew. Chem., Int. Ed. Engl. 1996, 35, 707.

(5) Feiters, M. C. In Comprehensive Supramolecular Chemistry; Atwood, J. L., Davies, J. E. D., MacNicol, D. D., Vögtle, F., Reinhoudt, D. N., Lehn, J.-M., Eds.; Elsevier Science Ltd., Pergamon: Elmsford, 1996; Vol. 10.

(6) Lehn, J.-M. Angew. Chem., Int. Ed. Engl. 1988, 27, 89.

(7) Cram, D. J. Angew. Chem., Int. Ed. Engl. 1988, 27, 1009.

(8) Pedersen, C. J. Angew. Chem., Int. Ed. Engl. 1988, 27, 1021.

(9) Cacciapaglia, R.; Di Stefano, S.; Kelderman, E.; Mandolini, L. Angew. Chem., Int. Ed. 1999, 38, 348.

(10) Wenz, G. Angew. Chem., Int. Ed. Engl. 1994, 33, 803.

(11) Breslow, R. Acc. Chem. Res. 1994, 28, 146.

(12) Breslow, R.; Dong, S. D. Chem. Rev. 1998, 98, 1997.

(13) Takahashi, K. Chem. Rev. 1998, 98, 2013.

(14) Breslow, R.; Schmuck, C. J. Am. Chem. Soc. 1996, 118, 6601

(15) Zhang, B. L.; Breslow, R. J. Am. Chem. Soc. 1997, 119, 1676

(16) Liu, S.; Luo, Z.; Hamilton, A. D. Angew. Chem., Int. Ed. Engl. 1997, 36, 2678 .

(17) Barr, L.; Easton, C. J.; Lee, K.; Lincoln, S. F.; Simpson, J. S Tetrahedron Lett. 2002, 42, 7797.

(18) Breslow, R.; Anslyn, E. J. Am. Chem. Soc. 1989, 111, 8931.

(19) Diederich, F.; Mattei, P. Helv. Chim. Acta 1997, 80, 1555.

(20) Diederich, F.; Habicher, T. Helv. Chim. Acta 1999, 82, 1066.

(21) Weber, L.; Imiolczyk, I.; Haufe, G.; Rehorek, D.; Hennig, H. J. Chem. Soc., Chem. Commun. 1992, 301.

(22) Weber, L.; Hommel, R.; Behling, J.; Haufe, G.; Hennig, H. J Am. Chem. Soc. 1994, 116, 2400 .

(23) Kuroda, Y.; Hiroshige, T.; Ogoshi, H. J. Chem. Soc., Chem. Commun. 1990, 1594.

(24) Kuroda, Y.; Sera, T.; Ogoshi, H. J. Am. Chem. Soc. 1991, 113, 2793.

(25) Bonar-Law, R. P.; Sanders, J. K. M. J. Chem. Soc., Chem. Commun. 1994, 474.

(26) Bonar-Law, R. P. J. Am. Chem. Soc. 1995, 117, 12397.

(27) Benson, D. R.; Valentekovich, R.; Diederich, F. Angew. Chem. 1990, 102, 213.

(28) Benson, D. R.; Valentekovich, R.; Knobler, C. B.; Diederich, F Tetrahedron 1991, 47, 2401.

(29) Benson, D. R.; Valentekovich, R.; Tam, S. W.; Diederich, F. Helv. Chim. Acta 1993, 76, 2034.

(30) Molenveld, P.; Engbersen, J. F. J.; Reinhoudt, D. N. Chem. Soc Rev. 2000, 29, 75 .

(31) Coolen, H. K. A. C.; van Leeuwen, P. W. N. M.; Nolte, R. J. M. Angew. Chem., Int. Ed. Engl. 1992, 31, 905.

(32) Coolen, H. K. A. C.; van Leeuwen, P. W. N. M.; Nolte, R. J. M. J. Am. Chem. Soc. 1995, 117, 11906.

(33) Elemans, J. A. A. W.; Bijsterveld, E. J. A.; Rowan, A. E.; Nolte, R. J. M. Chem. Commun. 2000, 2443.

(34) Thordarson, P.; Bijsterveld, E. J. A.; Rowan, A. E.; Nolte, R. J. M. Nature 2003, 424, 915.

(35) Scrimin, P.; Tecilla, P.; Tonellato, U.; Vignana, M. J. Chem. Soc., Chem. Commun. 1991, 449.

(36) Corey, E. J.; Guzman-Perez, A.; Noe, M. C. J. Am. Chem. Soc. 1994, 116, 12109 .

(37) Canary, J. W.; Allen, C. S.; Castagnetto, J. M.; Wang, Y. J. Am. Chem. Soc. 1995, 117, 8484 .

(38) Portade, T.; Roje, M.; Raza, Z.; Caplar, V.; Žinic, M.; Šunjic, V. Chem. Commun. 2000, 1993.

(39) Breslow, R.; Brown, A. B.; McCullogh, R. D.; White, P. W. J. Am. Chem. Soc. 1989, 111, 4517.

(40) Breslow, R.; Zhang, X.; Xu, R.; Maletic, M. J. Am. Chem. Soc. 1996, 118, 11678 .

(41) Breslow, R.; Huang, Y.; Zhang, X.; Yang, J. Proc. Natl. Acad. Sci. U.S.A. 1997, 94, 11156.

(42) Breslow, R.; Zhang, X.; Huang, Y. J. Am. Chem. Soc. 1997, 119 , 4535.

(43) Breslow, R.; Gabriele, B.; Yang, J. Tetrahedron Lett. 1998, 39 , 2887.

(44) Cacciapaglia, R.; Di Stefano, S.; Mandolini, L. Acc. Chem. Res 2004, 37, 113.

(45) Kim, S. P.; Leach, A. G.; Houk, K. N. J. Org. Chem. 2002, 67, 4250 .

(46) Wulff, G. Chem. Rev. 2002, 102, 1. 
(47) Mader, M.; Bartlett, P. A. Chem. Rev. 1997, 97, 1281

(48) Rideout, D. C.; Breslow, R. J. Am. Chem. Soc. 1980, 102, 7817.

(49) Breslow, R.; Guo, T. J. Am. Chem. Soc. 1988, 110, 5613.

(50) Schneider, H.-J.; Sangwan, N. K. J. Chem. Soc., Chem. Commun. 1986, 1787.

(51) Schneider, H.-J.; Sangwan, N. K. Angew. Chem., Int. Ed. Engl. 1987, 26,896

(52) Mock, W. L. Top. Curr. Chem. 1995, 175, 1.

(53) Kim, K. Chem. Soc. Rev. 2002, 31, 96

(54) Mock, W. L.; Irra, T. A.; Wepsiec, J. P.; Manimaran, T. L. J. Org. Chem. 1983, 48, 3619.

(55) Tuncel, D.; Steinke, J. H. G. Chem. Commun. 1999, 1509.

(56) Krasia, T. C.; Steinke, J. H. G. Chem. Commun. 2002, 22.

(57) Jon, S. Y.; Ko, Y. H.; Park, S. H.; Kim, H.-J.; Kim, K. Chem. Commun. 2001, 1938.

(58) Walter, C. J.; Anderson, H. L.; Sanders, J. K. M. J. Chem. Soc., Chem. Commun. 1993, 458.

(59) Clyde-Watson, Z.; Vidal-Ferran, A.; Twyman, L. J.; Walter, C J.; McCallien, D. W. J.; Fanni, S.; Bampos, N.; Wylie, R. S.; Sanders, J. K. M. New J. Chem. 1998, 493

(60) Marty, M.; Clyde-Watson, Z.; Twyman, L. J.; Nakash, M.; Sanders, J. K. M. Chem. Commun. 1998, 2265.

(61) Nakash, M.; Clyde-Watson, Z.; Feeder, N.; Davies, J. E.; Teat, S. J.; Sanders, J. K. M. J. Am. Chem. Soc. 2000, 122, 5286.

(62) Nakash, M.; Sanders, J. K. M. J. Org. Chem. 2000, 65, 7266.

(63) Mackay, L. G.; Wylie, R. S.; Sanders, J. K. M. J. Am. Chem. Soc. 1994, 116, 3141

(64) Cram, D. J.; Cram, J. M. Container Molecules and their Guests The Royal Society of Chemistry: Cambridge, 1994.

(65) Cram, D. J.; Tanner, M. E.; Thomas, R. E. Angew. Chem., Int. Ed. Engl. 1991, 30, 1024

(66) Warmuth, R. Angew. Chem., Int. Ed. Engl. 1997, 36, 1347.

(67) Warmuth, R. Chem. Commun. 1998, 59.

(68) Warmuth, R.; Marvell, M. A. Angew. Chem., Int. Ed. 2000, 39, 1117

(69) Warmuth, R.; Marvell, M. A. Chem.-Eur. J. 2001, 7, 1209.

(70) Ziegler, M.; Brumaghim, J. L.; Raymond, K. N. Angew. Chem., Int. Ed. 2000, 39, 4119

(71) Zyryanov, G. V.; Kang, Y.; Stampp, S. P.; Rudkevich, D. M. Chem. Commun. 2002, 2792

(72) Zyryanov, G. V.; Kang, Y.; Rudkevich, D. M. J. Am. Chem. Soc. 2003, 125, 2997

(73) Zyryanov, G. V.; Rudkevich, D. M. Org. Lett. 2003, 5, 1253

(74) Hof, F.; Craig, S. L.; Nuckolls, C.; Rebek, J., Jr. Angew. Chem., Int. Ed. 2002, 41, 1488

(75) Wang, L.; Vysotsky, O.; Bogdan, A.; Bolte, M.; Böhmer, V. Science 2004, 304, 1312

(76) Rebek, J., Jr. Chem. Soc. Rev. 1996, 25, 255

(77) Conn, M. M.; Rebek, J., Jr. Chem. Rev. 1997, 97, 1647.

(78) Rebek, J., Jr. Acc. Chem. Res. 1999, 32, 278.

(79) Hof, F.; Rebek, J., Jr. Proc. Natl. Acad. Sci. U.S.A. 2002, 99 , 4775

(80) Branda, N.; Wyler, R.; Rebek, J., Jr. Science 1994, 263, 1267.

(81) Mecozzi, S.; Rebek, J., Jr. Chem.-Eur. J. 1998, 4, 1016.

(82) Meissner, R. S.; Rebek, J., Jr.; de Mendoza, J. Science 1995, 270 , 1485

(83) Kang, J.; Rebek, J., Jr. Nature 1996, 382, 239.

(84) Kang, J.; Rebek, J., Jr. Nature 1997, 385, 50.

(85) Kang, J.; Hilmersson, G.; Santamaría, J.; Rebek, J., Jr. J. Am. Chem. Soc. 1998, 120, 3650

(86) Kang, J.; Santamaría, J.; Hilmersson, G.; Rebek, J., Jr. J. Am. Chem. Soc. 1998, 120, 7389.

(87) Chen, J.; Körner, S.; Craig, S. L.; Lin, S.; Rudkevich, D. M.; Rebek, J., Jr. Proc. Natl. Acad. Sci. U.S.A. 2002, 99, 2593.

(88) Heinz, T.; Rudkevich, D. M.; Rebek, J., Jr. Nature 1998, 394, 764.

(89) Chen, J.; Rebek, J., Jr. Org. Lett. 2002, 4, 327.

(90) Leininger, S.; Olenyuk, B.; Stang, P. J. Chem. Rev. 2000, 100 , 853

(91) Holliday, B. A.; Mirkin, C. A. Angew. Chem., Int. Ed. 2001, 40, 2022.

(92) Seidel, S. R.; Stang, P. J. Acc. Chem. Res. 2002, 35, 972.

(93) Sun, W.-Y.; Yoshizawa, M.; Kusukawa, T.; Fujita, M. Curr. Opin. Chem. Biol. 2002, 6, 757 .

(94) Pinalli, R.; Cristini, V.; Sottili, V.; Geremia, S.; Campagnolo, M.; Caneschi, A.; Dalcanale, E. J. Am. Chem. Soc. 2004, 126, 6516.

(95) Fujita, M. Chem. Soc. Rev. 1998, 27, 417.

(96) Fujita, M.; Oguro, D.; Miyazawa, M.; Oka, H.; Yamaguchi, K.; Ogura, K. Nature 1995, 378, 469 .

(97) Kusukawa, T.; Fujita, M. Angew. Chem., Int. Ed. 1998, 37, 3142.

(98) Kusukawa, T.; Fujita, M. J. Am. Chem. Soc. 1999, 121, 1397.

(99) Kusukawa, T.; Fujita, M. Angew. Chem., Int. Ed. 2001, 40, 1879.

(100) Kusukawa, T.; Fujita, M. J. Am. Chem. Soc. 2002, 124, 13576.

(101) Ito, H.; Kusukawa, T.; Fujita, M. Chem. Lett. 2000, 598.

(102) Yoshizawa, M.; Kusukawa, T.; Fujita, M.; Sakamoto, S.; Yamaguchi, K. J. Am. Chem. Soc. 2001, 123, 10454.

(103) Yoshizawa, M.; Takeyama, Y.; Kusukawa, T.; Fujita, M. Angew. Chem., Int. Ed. 2002, 41, 1347.
(104) Fujita, M.; Yu, S.-Y.; Kusukawa, T.; Funaki, H.; Ogura, K.; Yamaguchi, K. Angew. Chem., Int. Ed. 1998, 37, 2082.

(105) Yu, S.-Y.; Kusukawa, T.; Biradha, K.; Fujita, M. J. Am. Chem. Soc. 2000, 122, 2665.

(106) Yoshizawa, M.; Takeyama, Y.; Okano, T.; Fujita, M. J. Am. Chem. Soc. 2003, 125, 3243

(107) Heinz, T.; Rudkevich, D. M.; Rebek, J., Jr. Angew. Chem., Int Ed. 1999, 38, 1136

(108) Yoshizawa, M.; Kusukawa, T.; Fujita, M.; Yamaguchi, K. J. Am. Chem. Soc. 2000, 122, 6311.

(109) Merlau, M.; Del Pilar Mejia, M.; Nguyen, S. T.; Hupp, J. T. Angew. Chem., Int. Ed. 2001, 40, 4239.

(110) Slone, R. V.; Hupp, J. T. Inorg. Chem. 1997, 36, 5422.

(111) Mines, G. A.; Tzeng, B.-C.; Stevenson, K. J.; Li, J.; Hupp, J. T. Angew. Chem., Int. Ed. 2002, 41, 154.

(112) Slagt, V. F.; Reek, J. N. H.; Kamer, P. C. J.; van Leeuwen, P. W. N. M. Angew. Chem., Int. Ed. 2001, 40, 4271.

(113) Slagt, V. F.; Kamer, P. C. J.; van Leeuwen, P. W. N. M.; Reek, J. N. H. J. Am. Chem. Soc. 2004, 126, 1526.

(114) Chen, J.; Körner, S.; Craig, S. L.; Rudkevich, D. M.; Rebek, J., Jr. Nature 2002, 415, 385 .

(115) Otto, S.; Furlan, R. L. E.; Sanders, J. K. M. Science 2002, 297, 590.

(116) Brisig, B.; Sanders, J. K. M.; Otto, S. Angew. Chem., Int. Ed. 2003, 42, 1270 .

(117) Wulff, G.; Gross, T.; Schönfeld, R. Angew. Chem., Int. Ed. Engl. 1997, 36,1962

(118) Otto, S.; Furlan, R. L. E.; Sanders, J. K. M. J. Am. Chem. Soc. 2000, 122, 12063

(119) Alberts, B.; Bray, D.; Lewis, J.; Raff, M.; Roberts, K.; Watson, J. D. Molecular Biology of the Cell; Garland: New York, 1983

(120) Bangham, A. D.; Horne, R. W. J. Mol. Biol. 1964, 8, 660.

(121) Kunitake, T.; Okahata, Y. J. Am. Chem. Soc. 1977, 99, 3860.

(122) Tanford, C. The Hydrophobic Effect; Wiley-Interscience: New York, 1973.

(123) Blokzijl, W.; Engberts, J. B. F. N. Angew. Chem., Int. Ed. Engl. 1993, 32,1545 .

(124) For some recent reviews, see: (a) Furhhop, J.-H.; Köning, J. Membranes and Molecular Assemblies: The Synkinetic Approach; The Royal Society of Chemistry: Cambridge, 1994. (b) Löwik, D. W. P. M.; van Hest, J. C. M. Chem. Soc. Rev. 2004, 33, 234. (c) Velonia, K. Cornelissen, J. J. L. M.; Feiters, M. C. Rowan, A. E.; Nolte, R. J. M. Aggregation of Amphiphiles as a Tool to Create Novel Functional Nano-Objects. In Nanoscale Assemblies; Huck, W. T. S., Ed.; Kluwer Academic: New York, in press.

(125) Isrealachvilli, J. N.; Mitchell, D. J.; Ninham, B. W. J. Chem Soc., Faraday Trans. 2 1976, 72, 1525

(126) Isrealachvilli, J. N.; Marcelja, R. G.; R. G., H. Q. Rev. Soc. 1980 $13,121$.

(127) Häger, M.; Currie, F.; Holmberg, K. Top. Curr. Chem. 2003, 227 , 53

(128) Oehme, G. In Applied homogeneous catalysis with organometallic compounds: A comprehensive handbook in three volumes, 2nd ed.; Cornils, B., Ed.; VCH: Weinheim, 2002

(129) Bunton, C. A. In Kinetics and Catalysis in Microheterogeneous Systems; Grätzel, M., Kalyanasundaram, K., Eds.; Marcel Dekker: New York.

(130) Brown, J. M.; Baker, S. K.; Colens, A.; Darwent, J. R. In Enzymic and Nonenzymic Catalysis; Dunnil, P., Wiseman, A., Blakebrough, N., Eds.; Horwood: Chichester, 1980.

(131) Broos, J.; Visser, A. J. W. G.; Engbersen, J. F. J.; Verboom, W.; van Hoek, A.; Reinhoudt, D. N. J. Am. Chem. Soc. 1995, 117, 12657

(132) Tascioglu, S. Tetrahedron 1996, 52, 11113

(133) Lindstom, U. M. Chem. Rev. 2002, 102, 2751.

(134) Fendler, J. H.; Fendler, E. J. Catalysis in Micelles and Macromolecular Systems; Academic Press: London, 1975.

(135) Scrimin, P.; Tonellato, U. In Surfactants in Solution; Mittel, K L., Shah, D. D., Eds.; Plenum Press: New York, 1991; Vol. 11.

(136) A large body of work in this area using metallomicelles has been performed by Zeng and co-workers; see, for example: (a) Jiang, B. Y.; Xie, F.; Xie, J. Q.; Jiang, W. D.; Hu, C. W.; Zeng, X. C. J. Dispersion Sci. Technol. 2004, 25, 139. (b) Jiang, F. B.; J., D.; Yu, X. Q.; Bia, J. K.; Zeng, X. C. J. Colloid Interface Sci. 2004, 273, 497. (c) Zeng, X. C.; Zhang, Y.; Yu, X. Q.; Tia, A. Langmuir 1999, 15, 1621 and references cited.

(137) Harkins, W. D. J. Am. Chem. Soc. 1947, 69, 1428

(138) Menger, F. M.; Rhee, J. U.; Rhee, H. K. J. Org. Chem. 1975, 40, 3803 .

(139) Rabion, A.; Buchanan, R. M.; Seris, J.-L.; Fish, R. H. J. Mol. Catal. A: Chem. 1997, 116, 43.

(140) Oehme, G.; Grassert, I.; Paetzold, E.; Fuhrmann, H.; Dwars, T.; Schmidt, U.; Iovel, I. Kinet. Catal. 2003, 44, 766.

(141) Oehme, G.; Paetzold, E.; Selke, R. J. Mol. Catal. 1992, 71, L1.

(142) Grassert, I.; Paetzold, E.; Oehme, G. Tetrahedron 1993, 49, 6605

(143) Oehme, G.; Grassert, I.; Paetzold, E.; Meisel, R.; Drexler, K.; Fuhrmann, H. Coord. Chem. Rev. 1999, 186, 585

(144) Yonehare, K.; Ohe, K.; Uemura, S. J. Org. Chem. 1999, 64, 9381. 
(145) Ludwig, M.; Kadyrov, R.; Fiedler, H.; Haage, K.; Selke, R. Chem.Eur. J. 2001, 7, 3298

(146) Fuji, K.; Morimote, T.; Tsutsumi, K.; Kakiuchi, K. Angew. Chem., Int. Ed. 2003, 42, 2409.

(147) Kobayashi, S.; Manabe, K. Acc. Chem. Res. 2002, 35, 209.

(148) Kobayashi, S.; Wakabayashi, T.; Nagayama, S.; Oyamada, H. Tetrahedron Lett. 1997, 38, 4559.

(149) Kobayashi, S.; Wakabayashi, T. Tetrahedron Lett. 1998, 39, 5389.

(150) Manabe, K.; Mori, Y.; Wakabyashi, T.; Nagayama, S.; Kobayashi, S. J. Am. Chem. Soc. 2000, 122, 7202.

(151) Aoyama, N.; Manabe, K.; Kobayashi, S. Chem. Lett. 2004, 33, 312.

(152) Yates, P. Eaton, P. J. J. Am. Chem. Soc. 1960, 82, 4436.

(153) Breslow, R.; Rideout, D. C. J. Am. Chem. Soc. 1980, 102, 7816

(154) Otto, S.; Engberts, J. B. F. N.; Kwak, J. C. T. J. Am. Chem. Soc. 1998, 120,9517

(155) Diels-Alder reactions in micellar media, combined with solvent effect on the rate and stereochemistry of the reaction, have recently been reviewed: Otto, S.; Engberts, J. B. F. N. Surf. Sci. Ser. 2001, 100, 247.

(156) Gebicki, J. M.; Hicks, M. Nature 1973, 243, 232

(157) Pérez-Juste, J.; Hollfelder, F.; Kirby, A. J.; Engberts, J. B. F. N. Org. Lett. 2000, 2, 127.

(158) Klijn, J. E.; Engberts, J. B. F. N. J. Am. Chem. Soc. 2003, 125 , 1825.

(159) Klijn, J. E.; Engberts, J. B. F. N. Org. Biomol. Chem. 2004, 2 , 1789.

(160) Kimizuka, N.; Watanabe, E.; Kunitake, T. Chem. Lett. 1999, 29

(161) Rispens, T.; Engberts, J. B. F. N. Org. Lett. 2001, 3, 941.

(162) Groves, J.' T.; Han, Y. Z. In Cytochrome P450. Structure, Mechanism and Biochemistry; Ortiz de Montellano, P. R., Ed.; Plenum Press: New York, 1995.

(163) Mayer, J. M. Biomimetic Oxidations by Transition Metal Complexes; Imperial College Press: London, 2000.

(164) Mansuy, D. Pure Appl. Chem. 1994, 66, 737.

(165) Feiters, M. C.; Rowan, A. E.; Nolte, R. J. M. Chem. Soc. Rev. 2000, 29, 375 .

(166) Sorokin, A. B.; Khenkin, A. M.; Marakushev, S. A.; Shilov, A E.; Shteinman, A. A. Dokl. Phys. Chem. (Engl. Trans.) 1984, 29 1101

(167) Groves, J. T.; Neumann, R. J. Am. Chem. Soc. 1987, 109, 5045

(168) van Esch, J. H.; Roks, M. F. M.; Nolte, R. J. M. J. Am. Chem. Soc. 1986, 108, 6093

(169) Schenning, A. P. H. J.; Hubert, D. H. W.; van Esch, J. H.; Feiters, M. C.; Nolte, R. J. M. Angew. Chem., Int. Ed. Engl. 1994, 33, 2468 .

(170) Schenning, A. P. H. J.; Lutje Spelberg, J. H.; Hubert, D. H. W. Feiters, M. C.; Nolte, R. J. M. Chem.-Eur. J. 1998, 4, 871.

(171) Schenning, A. P. H. J.; Lutje Spelberg, J. H.; Driessen, M. C. P. F.; Hauser, M. J. B.; Feiters, M. C.; Nolte, R. J. M. J. Am. Chem. Soc. 1995, 117, 12655.

(172) Klein Gebbink, R. J. M.; Sandee, A. J.; Peters, F. G. A.; van der Gaast, S. J.; Feiters, M. C.; Nolte, R. J. M. J. Chem. Soc., Dalton Trans. 2001, 3056.

(173) Schreuder Goedheijt, M.; Hansen, B. E.; Reek, J. N. H.; Kamer, P. C. J.; van Leeuwen, P. W. N. M. J. Am. Chem. Soc. 2000, 122,1650

(174) Annesini, M. C. Chem. Biochem. Eng. Q. 1998, 12, 1.

(175) Sada, E.; Katoh, S.; Terashima, M.; Kheirolomoom, A.; Sawai, H. J. Chem. Eng. Jpn. 1987, 20,531.

(176) Kheirolomoom, A.; Katoh, S.; Sada, E.; Yoshida, K. Biotechnol. Bioeng. 1991, 37, 809.

(177) Monnard, P.-A. J. Membr. Biol. 2003, 191, 87.

(178) Walde, P.; Ichikawa, S. Biomol. Eng. 2001, 18, 143.

(179) Szostak, J. W.; Bartel, D. P.; Luisi, P. L. Nature 2001, 409, 387.

(180) Pohorille, A.; Deamer, D. W. Trends Biotechnol. 2002, 20, 123

(181) Oberholzer, T.; Albrizio, M.; Luisi, P. L. Chem. Biol. 1995, 2 677.

(182) Oberholzer, T.; Luisi, P. L. J. Biol. Phys. 2002, 28, 733.

(183) Schmidli, P.; Schurtenberger, P.; Luisi, P. L. J. Am. Chem. Soc. 1991, 113, 8127

(184) Bachmann, P. A.; Luisi, P. L.; Lang, J. Nature 1992, 357, 57.

(185) Walde, P.; Wick, R.; Fresta, M.; Mangone, A.; Luisi, P. L. J. Am. Chem. Soc. 1994, 116, 11649

(186) Walde, P.; Goto, A.; Monnard, P.-A.; Wessiken, M.; Luisi, P. L. J. Am. Chem. Soc. 1994, 116, 7541 .

(187) Chakrabarti, A. C.; Ereaker, R. R.; Joyce, G. F.; Deamer, D. W J. Mol. Evol. 1994, 39, 555.

(188) Treyer, M.; Walde, P.; Oberholzer, T. Langmuir 2002, 18, 1043.

(189) Oberholzer, T.; Meyer, E.; Amato, I.; Lustig, A.; Monnard, P.-A. Biochim. Biophys. Acta 1999, 1416, 57.

(190) Oberholzer, T.; Wick, R.; Luisi, P. L.; Biebricher, C. K. Biochem. Biophys. Res. Commun. 1995, 207, 250.

(191) Yu, W.; Wakabayashi, M.; Nakaishi, T.; Ko-Mitamura, E. P.; Shima, Y.; Urabe, I.; Yomo, T. J. Biosci. Bioeng, 2001, 92, 590

(192) Hill, K. J.; Kaszuba, M.; Creeth, J. E.; Jones, M. N. Biochim. Biophys. Acta 1997, 1326, 37.

(193) Kaszuba, M.; Jones, M. N. Biochim. Biophys. Acta 1999, 1419, 221.
(194) Jones, M. N.; Hill, K. J.; Kaszuba, M.; Creeth, J. E. Int. J. Pharm. 1998, 162, 107

(195) For some recent references in this area, see: (a) Yoshimoto, M. Wang, S. Q.; Fukunaga, K.; Walde, P.; Kuboi, R.; Nakao, K. Biotechnol. Bioeng. 2003, 81, 695. (b) Olea, D.; Faure, C. J. Chem. Phys. 2003, 119, 6111. (c) Rodiguez-Nogales, J. M. J. Chem. Technol. Biotechnol. 2004, 79, 72 .

(196) Menger, F. M.; Angelova, M. I. Acc. Chem. Res. 1998, 31, 789.

(197) Giant Vesicles; Luisi, P. L., Walde, P., Eds.; John Wiley \& Sons: Chichester, 2000.

(198) Fischer, A.; Franco, A.; Oberholzer, T. ChemBioChem 2002, 3, 409.

(199) Takakura, K.; Toyota, T.; Sugawara, T. J. Am. Chem. Soc. 2003 $125,8134$.

(200) Förster, S.; Antonietti, M. Adv. Mater. 1998, 10, 195.

(201) Park, C.; Yoon, J.; Thomas, E. L. Polymer 2003, 44, 6725.

(202) Förster, S.; Konrad, M. J. Mater. Chem. 2003, 13, 2671.

(203) Cameron, N. S.; Corbierre, M. K.; Eisenberg, A. Can. J. Chem. 1999, 77, 1311 .

(204) Discher, B. M.; Won, Y.-Y.; Ege, D. S.; Lee, J. C.-M.; Bates, F. S.; Discher, D. E.; Hammer, D. A. Science 1999, 284, 1143.

(205) Cornelissen, J. J. L. M.; Fischer, M.; Sommerdijk, N. A. J. M.; Nolte, R. J. M. Science 1998, 280, 1427.

(206) Jain, S.; Bates, F. S. Science 2003, 300, 460

(207) Jenekhe, S. A.; Chen, X. L. Science 1998, 279, 1903.

(208) van Hest, J. C. M.; Delnoye, D. A. P.; Baars, M. W. P. L.; van Genderen, M. H. P.; Meijer, E. W. Science 1995, 268, 1592.

(209) Zhang, L.; Eisenberg, A. Science 1995, 268, 1728.

(210) Discher, B. M.; Hammer, D. A.; Bates, F. S.; Discher, D. E. Curr Opin. Colloid Interface Sci. 2000, 5, 121

(211) Discher, D. E.; Eisenberg, A. Science 2002, 297, 967.

(212) Antonietti, M.; Förster, S. Adv. Mater. 2003, 15, 1323.

(213) Soo, P. L.; Eisenberg, A. J. Polym. Sci., Part B: Polym. Phys. $\mathbf{2 0 0 3}, 42,923$

(214) Aranda-Espinoza, H.; Bermudez, H.; Bates, F. S.; Discher, D. E. Phys. Rev. Lett. 2001, 87, 208301-1.

(215) Bermudez, H.; Brannan, A. K.; Hammer, D. A.; Bates, F. S. Discher, D. E. Macromolecules 2002, 35, 8203

(216) Discher, B. M.; Bermudez, H.; Hammer, D. A.; Discher, D. E. Won, Y.-Y.; Bates, F. S. J. Phys. Chem. B 2002, 106, 2848.

(217) Lee, J. C.-M.; Bermudez, H.; Discher, B. M.; Sheehan, M. A.; Won, Y.-Y.; Bates, F. S.; Discher, D. E. Biotechnol. Bioeng. 2001, 73,135 .

(218) Photos, P. J.; Bacakova, L.; Discher, B. M.; Bates, F. S.; Discher, D. E. J. Controlled Release 2003, 90,323.

(219) Napoli, A.; Boerakker, M. J.; Tirelli, N.; Nolte, R. J. M.; Sommerdijk, N. A. J. M.; Hubbell, J. A. Langmuir 2004, 20, 3487.

(220) Chécot, F.; Lecommandoux, S.; Klok, H.-A.; Gnanou, Y. Eur. Phys. J. E 2003, 10, 25.

(221) Bellomo, E. G.; Wyrsta, M. D.; Pakstis, L.; Pochan, D. J.; Deming, T. J. Nat. Mater. 2004, 3, 244

222) Najafi, F.; Sarbolouki, M. N. Biomaterials 2003, 24, 1175

(223) Nardin, C.; Thoeni, S.; Widmer, J.; Winterhalter, M.; Meier, W. Chem. Commun. 2000, 1433.

(224) Nardin, C.; Widmer, J.; Winterhalter, M.; Meier, W. Eur. Phys. J. E 2001, 4, 403

(225) Sauer, M.; Haefele, T.; Graff, A.; Nardin, C.; Meier, W. Chem. Commun. 2001, 2452.

(226) Graff, A.; Sauer, M.; van Gelder, P.; Meier, W. Proc. Natl. Acad. Sci. U.S.A. 2002, 99, 5064.

(227) Vriezema, D. M.; Hoogboom, J.; Velonia, K.; Takazawa, K. Christianen, P. C. M.; Maan, J. C.; Rowan, A. E.; Nolte, R. J. M. Angew. Chem., Int. Ed. 2003, 42, 772

(228) Vriezema, D. M.; Kros, A.; Hoogboom, J.; Rowan, A. E.; Nolte, R. J. M. Polym. Prepr. 2004, 45, 749 .

(229) White, S. R.; Sottos, N. R.; Geubelle, P. H.; Moore, J. S.; Kessler, M. R.; Sriram, S. R.; Brown, E. N.; Viswanathan, S. Nature 2001, 409, 794 .

(230) Boerakker, M. J.; Hannink, J. M.; Bomans, P. H. H.; Frederik, P. M.; Nolte, R. J. M.; Meijer, E. M.; Sommerdijk, N. A. J. M. Angew. Chem., Int. Ed. 2002, 41, 4239.

(231) Mayer, A. B. R. Polym. Adv. Technol. 2001, 12, 96

(232) Hamley, I. W. Nanotechnology 2003, 14, R39.

(233) Kastle, G.; Boyen, H. G.; Weigl, F.; Lengl, G.; Herzog, T.; Ziemann, P.; Riethmuller, S.; Mayer, O.; Hartmann, C.; Spatz, J. P.; Möller, M.; Ozawa, M.; Banhart, F.; Garnier, M. G. Oelhafen, P. Adv. Funct. Mater. 2003, 13, 853.

(234) Klingelhöfer, S.; Heitz, W.; Greiner, A.; Oestreich, S.; Förster S.; Antonietti, M. J. Am. Chem. Soc. 1997, 119, 10116.

(235) Spatz, J.; Mössmer, S.; Möller, M.; Kocher, M.; Neher, D.; Wegner, G. Adv. Mater. 1998, 10, 473

(236) Moffit, M.; McMahon, L.; Pessel, V.; Eisenberg, A. Chem. Mater. 1995, 7,1185

(237) Cornelissen, J. J. L. M.; van Heerbeek, R.; Kamer, P. C. J.; Reek, J. N. H.; Sommerdijk, N. A. J. M.; Nolte, R. J. M. Adv. Mater 2002, 14, 489 .

(238) Roucoux, A.; Schulz, J.; Patin, H. Chem. Rev. 2002, 102, 3757.

(239) Yu, W.; Liu, H.; Tao, Q. Chem. Commun. 1996, 1773. 
(240) Zuo, X.; Liu, H.; Liu, M. Tetrahedron Lett. 1998, 39, 1941.

(241) Harada, M.; Asakura, K.; Toshima, N. J. Phys. Chem. 1993, 97, 5103

(242) Shiraishi, Y.; Toshima, N. J. Mol. Catal. A: Chem. 1999, 141, 187.

(243) Shiraishi, Y.; Toshima, N. Colloids Surf., A 2000, 169, 59.

(244) Wang, Q.; Liu, H.; Han, M.; Li, X.; Jiang, D. J. Mol. Catal. A. Chem. 1997, 118, 145.

(245) Le Bars, J.; Specht, U.; Bradley, J. S.; Blackmond, D. G. Langmuir 1999, 15, 7621.

(246) Li, Y.; El-Sayed, M. A. J. Phys. Chem. B 2001, 105, 8938

(247) Lee, M.; Jang, C.-J.; Ryu, J.-H. J. Am. Chem. Soc. 2004, 126, 8082

(248) Naka, K.; Kubo, Y.; Ohki, A.; Maeda, S. Polym. J. 1994, 26, 243.

(249) Harada, A.; Kataoka, K. Macromolecules 1998, 31, 288.

(250) Harada, A.; Kataoka, K. Langmuir 1999, 15, 4208.

(251) Harada, A.; Kataoka, K. J. Am. Chem. Soc. 1999, 121, 9241.

(252) Harada, A.; Kataoka, K. J. Controlled Release 2001, 72, 85.

(253) Harada, A.; Kataoka, K. J. Am. Chem. Soc. 2003, 125, 15306.

(254) Velonia, K. Rowan, A. E.; Nolte, R. J. M. J. Am. Chem. Soc. 2002, 124,4224 .

(255) Jang, J.; Ha, H. Langmuir 2002, 18, 5613.

(256) Landfester, K. Colloid Chemistry II; Springer-Verlag: Berlin, 2003.

(257) Landfester, K.; Montenegro, R.; Scherf, U.; Güntner, R.; Asawapirom, U.; Patil, S.; Neher, D.; Kietzke, T. Adv. Mater. 2002 14,651 .

(258) Tiarks, F.; Landfester, K.; Antonietti, M. Langmuir 2001, 17, 908.

(259) Bosman, A. W.; Janssen, H. M.; Meijer, E. W. Chem. Rev. 1999, 99, 1665

(260) Crooks, R. M.; Zhao, M.; Sun, L.; Chechik, V.; Yeung, L. K. Acc. Chem. Res. 2001, 34, 181.

(261) Heerbeek, R.; Kamer, P. C. J.; van Leeuwen, P. W. N. M.; Reek, J. N. H. Chem. Rev. $2002,102,3717$.

(262) Twyman, L. J.; King, A. S. H.; Martin, I. K. Chem. Soc. Rev. $2002,31,69$

(263) Fréchet, J. M. J. Macromol. Symp. 2003, 201, 11.

(264) Fréchet, J. M. J. J. Polym. Sci., Part A: Polym. Chem. 2003, 41,3713

(265) Caminade, A.-M.; Turrin, C.-O.; Sutra, P.; Majoral, J.-P. Curr Opin. Colloid Interface Sci. 2003, 8, 282.

(266) Sunder, A.; Hanselmann, R.; Frey, H.; Mülhaupt, R. Macromolecules 1999, 32,4240

(267) Youk, J. H.; Park, M.-K.; Locklin, J.; Advincula, R.; Yang, J.; Mays, J. Langmuir 2002, 18, 2455.

(268) Bosman, A. W.; Vestberg, R.; Heumann, A.; Fréchet, J. M. J.; Hawker, C. J. J. Am. Chem. Soc. 2003, 125, 715.

(269) Mecking, S.; Thomann, R.; Frey, H.; Sunder, A. Macromolecules 2000, 33, 3958

(270) Slagt, M. Q.; Stiriba, S.-E.; Klein Gebbink, R. J. M.; Kautz, H.; Frey, H.; van Koten, G. Macromolecules 2002, 35, 5734.

(271) Terashima, T.; Kamigaito, M.; Baek, K.-Y.; Ando, T.; Sawamoto, M. J. Am. Chem. Soc. 2003, 125, 5288.

(272) Chernyshov, D. M.; Bronstein, L. M.; Börner, H.; Berton, B.; Antonietti, M. Chem. Mater. 2000, 12, 114

(273) Jungmann, N.; Schmidt, M.; Maskos, M. Macromolecules 2003 36,3974

(274) Donath, E.; Sukhorukov, G. B.; Caruso, F.; Davis, S. A.; Möhwald, H. Angew. Chem., Int. Ed. 1998, 37, 2201.

(275) Decher, G. Science 1997, 277, 1232.

(276) Caruso, F.; Caruso, R. A.; Möhwald, H. Science 1998, 282, 1111

(277) Dähne, L.; Leporatti, S.; Donath, E.; Möhwald, H. J. Am. Chem. Soc. 2001, 123, 5431

(278) Peyratout, C. S.; Möhwald, H.; Dähne, L. Adv. Mater. 2003, 15, 1722.

(279) Donath, E.; Moya, S.; Neu, B.; Sukhorukov, G. B.; Georgieva, R.; Voigt, A.; Baümler, H.; Kiesewetter, H.; Möhwald, H. Chem. Eur. J. 2002, 8, 5481 .

(280) Antipov, A.; Shchukin, D.; Fedutik, Y.; Zanaveskina, I.; Klechkovskaya, V.; Sukhorukov, G. B.; Möhwald, H. Macromol. Rapid Commun. 2003, 24, 274.

(281) Ghan, R.; Shutava, T.; Patel, A.; John, V. T.; Lvov, Y. Macromolecules 2004, 37, 4519.

282) Chang, T. M. S. J. Intern. Med. 2003, 253, 527.

(283) Chang, T. M. S.; Powanda, D.; Yu, W. P. Artif. Cells, Blood Substitutes, Immobilization Biotechnol. 2003, 31, 231.

(284) Lu, Z.-L.; Lindner, E.; Mayer, H. A. Chem. Rev. 2002, 102, 3543.

(285) Gill, I. Chem. Mater. 2001, 13, 3404.

(286) Jin, W.; Brennan, J. D. Anal. Chim. Acta 2002, 461, 1.

(287) Corma, A.; Garcia, H. Eur. J. Inorg. Chem. 2004, 1143

(288) Herron, N. Inorg. Chem. 1986, 25, 4714.

(289) Balkus, K. J., Jr.; Khanmamedova, A. K.; Dixon, K. M.; Bedioui, F.; Roué, L.; Devynck, J. Inorg. Chem. 1994, 33, 67.

(290) Zhan, B.-Z.; Li, X.-Y. Chem. Commun. 1998, 349.

(291) Parton, F. R.; Vankelecom, I. F. J.; Casselman, M. J. A.; Bezoukhanova, C. P.; Uytterhoeven, L.; Jacobs, P. A. Nature 1994, 370,541 .
(292) Kahlen, W.; Janssen, A.; Holderich, W. F. Stud. Surf. Sci. Catal. 1997, 108,469

(293) Sulikowski, B.; Haber, J.; Kubacka, A.; Pamin, K.; Olejniczak, Z.; Ptaszynski, J. Catal. Lett. 1996, 39, 27.

(294) Abrahams, B. F.; Hoskins, B. F.; Michail, D. M.; Robson, R. Nature 1994, 369, 727 .

(295) Venkataraman, D.; Lee, S.; Zhang, J. S.; Moore, J. S. Nature 1994, 371,591 .

(296) Endo, K.; Sawaki, T.; Koyanagi, M.; Kobayashi, K.; Masuda, H. Aoyama, Y. J. Am. Chem. Soc. 1995, 117, 8341 .

(297) Russell, V. A.; Evans, C. C.; Li, W. J.; Ward, M. D. Nature 1997 $276,575$.

(298) Kobayashi, K.; Shirasaka, T.; Sato, A.; Horn, E.; Furukawa, N. Angew. Chem., Int. Ed. 1999, 38, 3483.

(299) Matsumoto, A.; Odani, T.; Sada, K.; Miyata, M.; Tashiro, K. Nature 2000, 405, 328.

(300) Miller, S. A.; Kim, E.; Gray, D. H.; Gin, D. L. Angew. Chem., Int. Ed. 1999, 38, 3022.

(301) Dewa, T.; Saiki, T.; Imai, Y.; Endo, K.; Aoyama, Y. Bull. Chem. Soc. Jpn. 2000, 73, 2123

(302) Tajima, K.; Aida, T. Chem. Commun. 2000, 2399.

(303) Douglas, T.; Young, M. Adv. Mater. 1999, 11, 679

(304) Niemeyer, C. M. Angew. Chem., Int. Ed. 2001, 40, 4128

(305) Flynn, C. E.; Lee, S.-W.; Peelle, B. R.; Belcher, A. M. Acta Mater. 2003, $51,5867$.

306) Arora, P. S.; Kirshenbaum, K. Chem. Biol. 2004, 11, 418

(307) Mao, C. B.; Solis, D. J.; Reiss, B. D.; Kottmann, S. T.; Sweeney, R. Y.; Hayhurst, A.; Georgiou, G.; Iverson, B.; Belcher, A. M. Science 2004, 303, 213

(308) Chasteen, N. D.; Harrison, P. M. J. Struct. Biol. 1999, 126, 182

(309) Meldrum, F. C.; Wade, V. J.; Nimmo, D. L.; Heywood, B. R.; Mann, S. Nature 1991, 349, 684.

(310) Mann, S.; Meldrum, F. C. Adv. Mater. 1991, 3, 316

(311) Douglas, T.; Dickson, D. P. E.; Betteridge, S.; Charnock, J.; Garner, C. D.; Mann, S. Science 1995, 269, 54.

(312) Meldrum, F. C.; Douglas, T.; Levi, S.; Arosio, P.; Mann, S. J. Inorg. Biochem. 1995, 58, 59 .

(313) Douglas, T.; Stark, V. T. Inorg. Chem. 2000, 39, 1828.

(314) Meldrum, F. C.; Heywood, B. R.; Mann, S. Science 1992, 257 522.

(315) Wong, K. K. W.; Douglas, T.; Gider, S.; Awschalom, D. D.; Mann, S. Chem. Mater. 1998, 10, 279.

(316) Kim, I.; Hosein, H.-A.; Strongin, D. R.; Douglas, T. Chem. Mater. 2002, 14, 4874

(317) Ensign, D.; Young, M.; Douglas, T. Inorg. Chem. 2004, 43, 3441

(318) Ueno, T.; Suzuki, M.; Goto, T.; Matsumoto, T.; Nagayama, K.; Watanabe, Y. Angew. Chem., Int. Ed. 2004, 43, 2527.

(319) Wong, K. K. W.; Cölfen, H.; Whilton, N. T.; Douglas, T.; Mann, S. J. Inorg. Biochem. 1999, 76, 187

(320) Li, M.; Wong, K. K. W.; Mann, S. Chem. Mater. 1999, 11, 23.

(321) Yang, X.; Chiancone, E.; Stefanini, S.; Ilari, A.; Chasteen, N. D. Biochem. J. 2000, 349, 783.

(322) Allen, M.; Willits, D.; Mosolf, J.; Young, M.; Douglas, T. Adv. Mater. 2002, 14, 1562

(323) Allen, M.; Willits, D.; Young, M.; Douglas, T. Inorg. Chem. 2003 42,6300 .

(324) Shenton, W.; Mann, S.; Cölfen, H.; Bacher, A.; Fischer, M. Angew. Chem., Int. Ed. 2001, 40, 442.

(325) Flenniken, M. L.; Willits, D. A.; Brumfield, S.; Young, M.; Douglas, T. Nano Lett. 2003, 3, 1573

(326) Shenton, W.; Douglas, T.; Young, M.; Stubbs, G.; Mann, S. Adv. Mater. 1999, 11, 253.

(327) Knez, M.; Sumser, M. P.; Bittner, A. M.; Wege, C.; Jeske, H. Kooi, S.; Burghard, M.; Kern, K. J. Electroanal. Chem. 2002 522,70 .

(328) Knez, M.; Bittner, A. M.; Boes, F.; Wege, C.; Jeske, H.; Mai, E.; Kern, K. Nano Lett. 2003, 3, 1079.

(329) Dujardin, E.; Peet, C.; Stubbs, G.; Culver, J. N.; Mann, S. Nano Lett. 2003, 3, 413

(330) Fujikawa, S.; Kunitake, T. Langmuir 2003, 19, 6545

(331) Nedoluzhko, A.; Douglas, T. J. Inorg. Biochem. 2001, 84, 233.

(332) Fowler, C. E.; Shenton, W.; Stubbs, G.; Mann, S. Adv. Mater. 2001, 13, 1266

(333) Knez, M.; Sumser, M.; Bittner, A. M.; Wege, C.; Jeske, H. Hoffmann, D. M. P.; Kuhnke, K.; Kern, K. Langmuir 2004, 20, 441.

(334) Flynn, C. E.; Mao, C. B.; Hayhurst, A.; Williams, J. L.; Georgiou, G.; Iverson, B.; Belcher, A. M. J. Mater. Chem. 2003, 13, 2414

(335) Whaley, S. R.; English, D. S.; Hu, E. L.; Barbara, P. F.; Belcher, A. M. Nature 2000, 405, 665 .

(336) Lee, S.-W.; Mao, C. B.; Flynn, C. E.; Belcher, A. M. Science 2002 296,892

(337) Reiss, B. D.; Mao, C.; Solis, D. J.; Ryan, K. S.; Thomson, T.; Belcher, A. M. Nano Lett. 2004, 4, 1127.

(338) Mao, C. B.; Flynn, C. E.; Hayhurst, A.; Sweeney, R.; Qi, J. F.; Georgiou, G.; Iverson, B.; Belcher, A. M. Proc. Natl. Acad. Sci. U.S.A. 2003, 100, 6946.

(339) Lee, S.-W.; Lee, S. K.; Belcher, A. M. Adv. Mater. 2003, 15, 689. 
(340) Nam, K. T.; Peelle, B. R.; Lee, S. W.; Belcher, A. M. Nano Lett. 2004, 4, 23.

(341) Lee, S. W.; Wood, B. M.; Belcher, A. M. Langmuir 2003, 19, 1592

(342) Lee, S. W.; Belcher, A. M. Nano Lett. 2004, 4, 387.

(343) Wang, Q.; Lin, T.; Tang, L.; Johnson, J. E.; Finn, M. G. Angew. Chem., Int. Ed. 2002, 41, 459.

(344) Wang, Q.; Kaltgrad, E.; Lin, T.; Johnson, J. E.; Finn, M. G. Chem. Biol. 2002, 9, 805.

(345) Wang, Q.; Lin, T. W.; Johnson, J. E.; Finn, M. G. Chem. Biol. 2002, 9, 813 .

(346) Raja, K. S.; Wang, Q.; Gonzalez, M. J.; Manchester, M.; Johnson, J. E.; Finn, M. G. Biomacromolecules 2003, 4, 472.

(347) Wang, Q.; Raja, K. S.; Janda, K. D.; Lin, T.; Finn, M. G. Bioconjugate Chem. 2003, 14, 38.

(348) Wang, Q.; Chan, T. R.; Hilgraf, R.; Fokin, V. V.; Sharpless, K. B.; Finn, M. G. J. Am. Chem. Soc. 2003, 125, 3192.

(349) Raja, K. S.; Wang, Q.; Finn, M. G. ChemBioChem 2003, 4, 1348

(350) Blum, A. S.; Soto, C. M.; Wilson, C. D.; Cole, J. D.; Kim, M.; Gnade, B.; Chatterji, A.; Ochoa, W. F.; Lin, T.; Johnson, J. E.; Ratna, B. R. Nano Lett. 2004, 4, 867.

(351) Meunier, S.; Strable, E.; Finn, M. G. Chem. Biol. 2004, 11, 319.
(352) Strable, E.; Johnson, J. E.; Finn, M. G. Nano Lett. 2004, 4, 1385.

(353) Fang, J. Y.; Soto, C. M.; Lin, T. W.; Johnson, J. E.; Ratna, B. Langmuir 2002, 18, 308.

(354) Cheung, C. L.; Camarero, J. A.; Woods, B. W.; Lin, T.; Johnson, J. E.; De Yoreo, J. J. J. Am. Chem. Soc. 2003, 125, 6848.

(355) Smith, J. C.; Lee, K. B.; Wang, Q.; Finn, M. G.; Johnson, J. E.; Mrksich, M.; Mirkin, C. A. Nano Lett. 2003, 3, 883.

(356) Gillitzer, E.; Willits, D.; Young, M.; Douglas, T. Chem. Commun 2002, 2390.

(357) Hooker, J. M.; Kovacs, E. W.; Francis, M. B. J. Am. Chem. Soc. 2004, 126, 3718.

(358) Speir, J. A.; Munshi, S.; Wang, G.; Baker, T. S.; Johnson, J. E. Structure 1995, 3, 63.

(359) Johnson, J. E.; Speir, J. A. J. Mol. Biol. 1997, 269, 665.

(360) Douglas, T.; Young, M. Nature 1998, 393, 152.

(361) Douglas, T.; Strable, E.; Willits, D.; Aitouchen, A.; Libera, M.; Young, M. Adv. Mater. 2002, 14, 415.

(362) Klem, M. T.; Willits, D.; Young, M.; Douglas, T. J. Am. Chem. Soc. 2003, 125, 10806.

CR0300688 
